\title{
Photosystem II based Photoelectrochemical Cell
}

\author{
Mark Zolkiewski, B. Sc
}

A thesis submitted to the Department of

Chemistry in partial fulfillment of the

requirements for the degree of

Masters of Science in Chemistry

\author{
Department of Chemistry \\ Carleton University \\ Ottawa, Ontario, Canada
}

(C) 2017, Mark Zolkiewski 


\begin{abstract}
The need for renewable sources of electrical power continues to grow around the globe, with solar energy at the forefront. This thesis concerns the assembly and testing of a photoelectrochemical cell that utilizes photosynthetic proteins found in plants to capture solar energy and generate electricity. Photosystem II, plastoquinone and thylakoid lipids were extracted from spinach leaves for use in the photocell. An $\omega$-Hydroxythiol SAM on Au electrode was used as the anode to receive electrons from cytochrome $\mathrm{C}$ in solution. A platinum on carbon electrode was used as the cathode, with an anion exchange membrane to avoid short circuits forming between the electrodes. The photocell was tested under a variety of conditions and the highest photocurrent produced was $11.5 \mu \mathrm{A} / \mathrm{cm}^{2}$ at an applied voltage of $750 \mathrm{mV}$.
\end{abstract}




\section{Acknowledgements}

I would like to thank most of all my supervisors Steven McGarry and Sean Barry for giving me the opportunity to pursue this line of research. I want to thank Steven McGarry in particular for having infinite patience and providing invaluable advice throughout my degree.

I would like to thank Jeffrey Manthorpe for allowing me to use his lab, as well as all the members of the Manthorpe lab for all their advice and assistance. I also need to thank Shelley Hepworth for allowing me access to her lab. I want to thank all the members of the Smith and Wang lab in Chemistry who provided me with assistance and advice. I also want to thank Claudia Buttera, James Cheetham and Nagui Mikhail for providing me with technical assistance. Lastly I want to thank anyone in the Chemistry, Biology or Electronics departments that helped me in anyway, this research would not have been possible without their help.

Finally I want to dedicate this work to my late friend Adrian Dudzicki, this one's for you. 


\section{Table of Contents}

1 Introduction $\quad 1$

1.1 Overview of Solar Power Technology . . . . . . . . . . . . . . . . . . 1

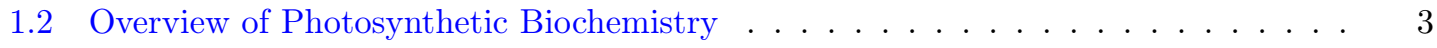

1.2.1 Photosynthetic Electron Transport Chain . . . . . . . . . . . . . . 3

$1.2 .2 \quad$ Photosystem II . . . . . . . . . . . . . . . . . . . . . . . 6

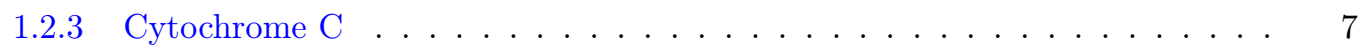

1.2.4 Thylakoid Membrane and Lipid Dynamics . . . . . . . . . . . . . . . 10

1.3 Chromatography . . . . . . . . . . . . . . . . . . . . . . . . 12

1.4 Electrochemistry . . . . . . . . . . . . . . . . . . . . 14

1.4.1 Oxygen Reduction Reaction . . . . . . . . . . . . . . . . . . . . . 14

1.4 .2 Cyclic Voltammetry . . . . . . . . . . . . . . . . . . . 15

1.5 Literature Review . . . . . . . . . . . . . . . . . . . . . . 16

1.6 Photoelectrochemical Cell Design . . . . . . . . . . . . . . . . . . . . . . . . 22

2 Extraction Production and Testing of PEC Components 25

2.1 Thylakoid Extraction . . . . . . . . . . . . . . . . . . . . . 25

2.2 Photosystem II Extraction . . . . . . . . . . . . . . . . . . . . . 27

$2.2 .1 \quad$ SDS-PAGE Analysis . . . . . . . . . . . . . . . . . . . . . . . 29

2.2 .2 Photosystem II Activity and Oxygen Evolution . . . . . . . . . . . . . . 31

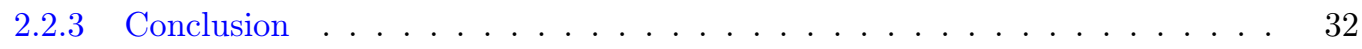


2.3 Plastoquinone Extraction $\ldots \ldots \ldots \ldots \ldots \ldots$

2.3.1 Plastoquinone NMR Analysis . . . . . . . . . . . . . . . . . . . . . . . 34

2.3.2 Plastoquinone Quantitative Analysis . . . . . . . . . . . . . . . . . . . 37

2.4 Thylakoid Lipid Extraction _ . . . . . . . . . . . . . . . . . . . . . . 37

2.4 .1 Thylakoid Lipid NMR Analysis . . . . . . . . . . . . . . . . . . . . 39

$2.5 \omega$-Hydroxythiol SAM Anode Production . . . . . . . . . . . . . . . 41

2.6 Anion Exchange Membrane Production _ . . . . . . . . . . . . . . . . . . 42

2.6.1 Anion Exchange Membrane ATR-IR Analysis . . . . . . . . . . . . . . . . 44

2.7 Platinum on Carbon Cathode Production . . . . . . . . . . . . . . . . 45

2.8 Cyclic Voltammetry Analysis _ . . . . . . . . . . . . . . . . . . . . . . 46

2.8.1 Anode Cyclic Voltammetry Analysis . . . . . . . . . . . . . . . . . . 46

2.8.2 Cathode Cyclic Voltammetry Analysis . . . . . . . . . . . . . . . 48

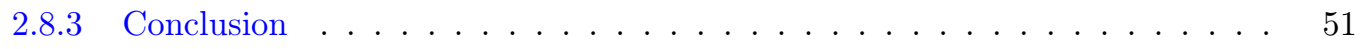

2.9 Plastoquinone and Cytochrome $\mathrm{C}$ redox Activity $\ldots \ldots \ldots \ldots$. . . . . . 51

3 Photoelectrochemical Cell Assembly and Testing 53

3.1 Photoelectrochemical Cell Results _. . . . . . . . . . . . . . . . . 55

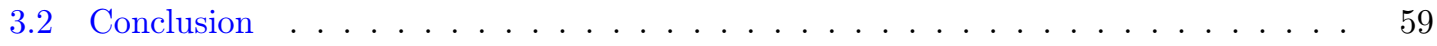




\section{Nomenclature}

$d \mathrm{H}_{2} \mathrm{O}$ Distilled water

$g \quad$ Acceleration due to gravity

ADP Adenosine diphosphate

AM Air-mass

ATP Adenosine triphosphate

ATR-IR Attenuated total reflection infrared spectroscopy

BOD Bilirubin oxidase

Cc Cytochrome C

CV Cyclic voltammetry

DCBQ 2,6-dichloro-p-benzoquinone

DGDG Digalactosyldiacylglycerol

DI de-ionized water

DSSC Dye-sensitized solar cell

EDTA Ethylenediaminetetraacetic acid

ET Electron transfer

FTO Fluorine-doped tin oxide

GA Glutaraldehyde

GDP Gross domestic product

HEPES 4-(2-hydroxyethyl)-1-piperazineethanesulfonic acid

J Joules

KPS Potassium phosphate buffer 
LHC Light harvesting complex

MGDG Monogalactosyldiacylglycerol

MWNT Multi-walled carbon nanotube

NADP / NADPH Nicotinamide adenine dinucleotide phosphate

NMR Nuclear magnetic resonance

OEC Oxygen evolving complex

ORR Oxygen reduction reaction

PBSE 1-pyrenebutanoic acid succinimidyl ester

PDDA Polydiallyldimethylammonium chloride

PEC Photoelectrochemical cell

PEDOT:PSS poly(3,4-ethylenedioxythiophene) polystyrene sulfonate

PG Phosphatidylglycerol

PMSF Phenylmethylsulfonyl fluoride

PQ Plastoquinone or plastoquinol

PSI Photosystem I protein complex

PSII Photosystem II protein complex

$\mathrm{Pt} / \mathrm{C}$ Platinum on graphitized carbon

pTLC Preperatory thin layer chromatography

PVA Polyvinyl alcohol

Rf Retention factor

SAM Self assembled monolayer

SDS-PAGE Sodium dodecyl sulfate polyacrylamide gel electrophoresis 
SQDG Sulfoquinovosyl diacylglycerol

SWCNT Single walled carbon nanotube

TLC Thin layer chromatography

TMS Tetramethylsilane

UV-Vis Ultraviolet-visible

Yz Tyrosine in D1 protein of photosystem II involved in oxygen evolution 
OECD gross electricity production by source, 1974-2016

Electricity Information 2017

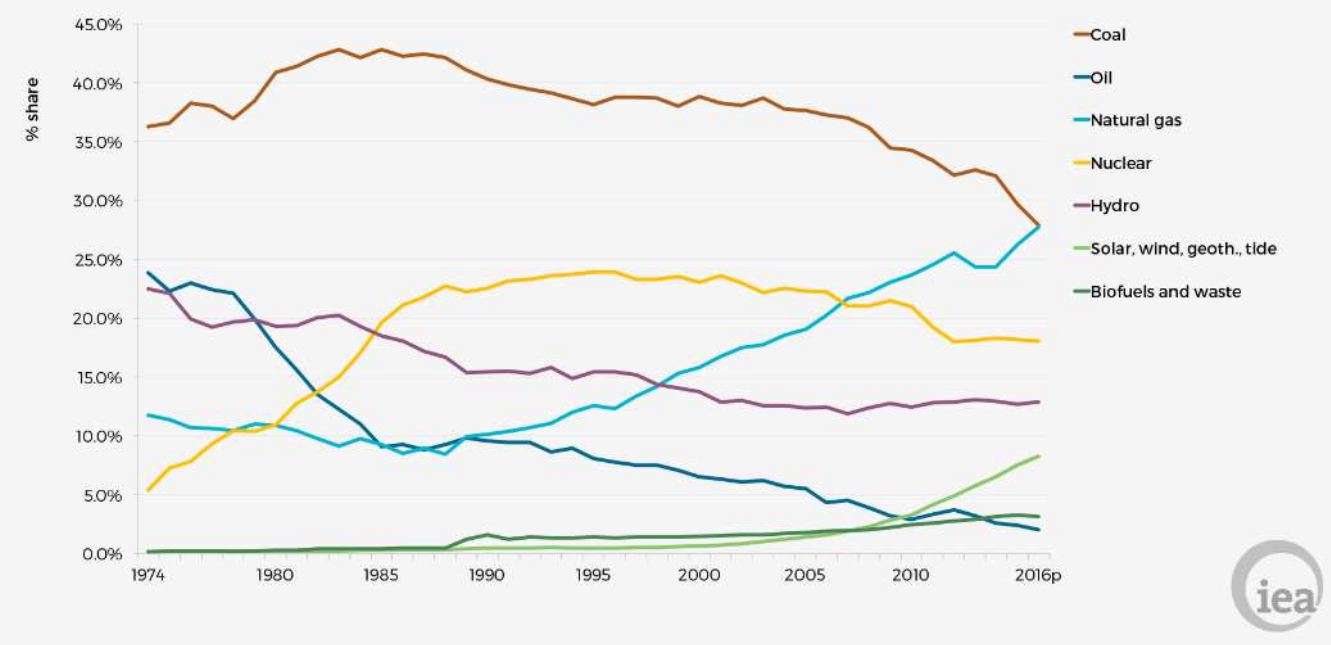

Figure 1: Global Energy Production by Source for 1974-2016.[1]

\section{Introduction}

\subsection{Overview of Solar Power Technology}

As the standard of living and the population of earth continualy rises, the need for inexpensive electrical power increases as well. Combined with diminishing fossil fuel reserves and growing concerns about the environmental impact of carbon emissions, the need for abundant renewable energy is greater than ever. The last ten years have seen a steady increase in the electrical energy production from renewable sources, as seen below in Figure 1.[1] According to recent data from US Energy Information Administration renewable sources of electrical energy, excluding hydroelectric, account for 9.4\% of global production in 2017 as of August. [2] This data also showed that solar accounts for $1.8 \%$ of global production, an increase of over $40 \%$ from the previous year. The surface of the earth is annually bombarded with $3.85 \times 10^{24} \mathrm{~J}$ of energy from the sun [3], Tapping into just $1 \%$ of this would equal three times our annual electrical energy consumption. Solar energy appears to be an obvious candidate as we try to find alternatives to coal and natural gas based power generation. The vast majority of current solar energy comes from photovoltaic devices. This body of work however attempts to develop a new approach to solar energy capture, using the primary photosynthetic proteins found in higher plants and algae in order to capture solar energy 
and convert it into electricity.

Conventional solar cells use semiconducting materials that are doped with specific elements in order to produce a p-n junction.[4] Silicon is the most common semiconductor used, with elements such as boron and phosphorous used for $\mathrm{p}$ and $\mathrm{n}$ type doping respectively. Simply put, a $\mathrm{p}$ type semiconductor has an abundance of holes while an $\mathrm{n}$ type semiconductor has an abundance of free electrons. When two such surfaces are placed in contact with each other they form a p-n junction, as seen in Figure 2. When a semiconductor such as silicon is bombarded with photons of sufficient energy, an excited electron is produced and a hole is left behind. These conducting electrons in the n-type semiconductor travel to the front contact where they are collected and run through an external load, while the holes travel to the p-type semiconductor and then to the rear contact where they recombine with the electrons and the "hole" is filled.

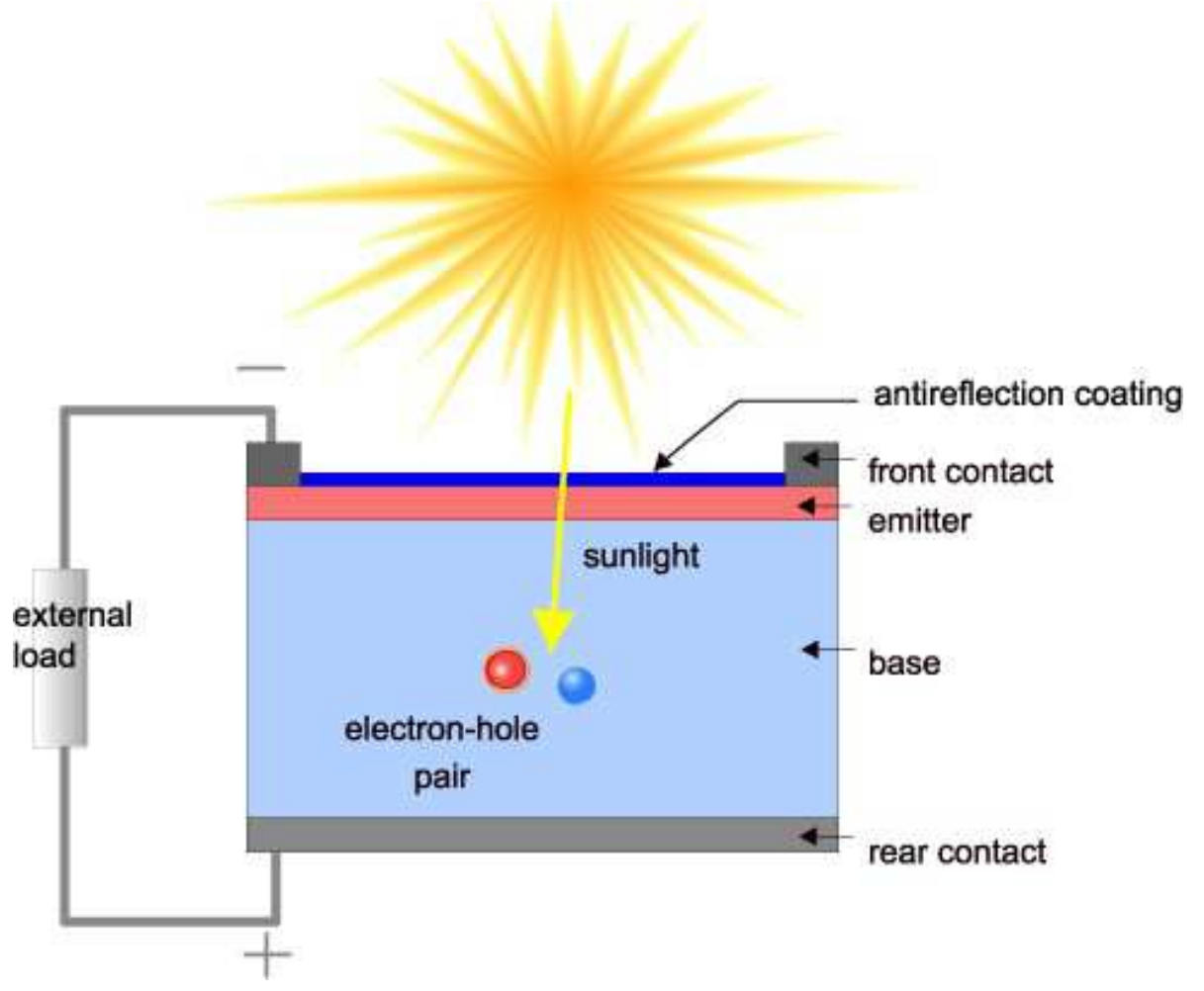

Figure 2: Shows a simplified diagram of a PN-Junction Photocell.[5]

In order to assess the feasibility of solar energy we need to look at the economic requirements for power generation, namely the maximum allowable cost of production and installation of solar cells. By taking the global annual GDP allotted to power generation and simply dividing it by the worldwide energy consumptions we arrive at a value of $\$ 15 /$ GJ, the approximate price point 
of electrical energy. [6] This price is equivalent to roughly $\$ 92$ for a barrel of oil. Assuming an ideal location, such as Sonoma California, one square meter of land is bombarded annually with 6.3 GJ of solar energy which is equivalent to an average power density of $200 \mathrm{~W} / \mathrm{m}^{2}$. If solar cells were $100 \%$ efficient in converting sunlight into electricity, the price point for the system would need to be less then $\$ 500 / m^{2}$. However solar cells are nowhere near this efficient, most commercial available solar cells have an annual efficiency of roughly $15 \%$. Assuming a rather conservative overage cost of $5 \%$ of the energy product to cover upkeep, and an additional $25 \%$ to cover installation and component costs not related to the solar cell itself, we arrive at a price point of $\$ 54 / \mathrm{m}^{2}$. The price of a solar power generation system in 2012 was roughly $\$ 750 / \mathrm{m}^{2}$, which would require an efficiency of $208 \%$ in order to be economically viable. The price of solar cells has dropped considerably since 2012, with utility scale solar energy plants reaching the $\$ 1 /$ watt according to the NREL.[7] This is however based on a model using using many assumptions, for example; that the photocells are operating at peak wattage instead of their yearly average, that the plant is supplying DC power and that the entire endeavor is heavily subsized. The average USD / watt cost could easily be close to $\$ 5 /$ watt [6] meaning the price would still need to drop significantly in order to be economically competitative with other sources of electricity.

These numbers make it very clear, that in order to solar cell technology to compete with other sources of primary power, a radical change in the technology needs to take place.

\subsection{Overview of Photosynthetic Biochemistry}

\subsubsection{Photosynthetic Electron Transport Chain}

Photosynthesis is the process by which plants and other organisms capture light energy and convert it into chemical energy to be used as fuel for the organism. Pictured in Figure 3, an average plant cell is roughly $50 \mu \mathrm{m}$ in diameter and can contain up to 100 chloroplasts in its cytoplasm.[8] Chloroplasts are roughly $5 \mu \mathrm{m}$ in diameter and house the photosynthetic electron transport chain inside small membrane bound compartments called thylakoids. These thylakoid membranes are organized in interconnected stacks called grana. Within the lipid membrane of these thylakoid sits 
the proteins that participate in the photosynthetic electron transport chain. The primary function of this biochemical pathway is to convert light energy into an electrochemical gradient of protons across the lipid membrane of the thylakoid. Proton concentration builds up in the lumen of the thylakoid, the membrane bound compartment inside the thylakoid, and these protons then diffuse across the membrane through the protein ATP synthase, driving the production of ATP from ADP. This process is called photophosphorylation. Adenosine triphosphate (ATP) is the primary source of chemical energy for the cell metabolism in most living organisms.
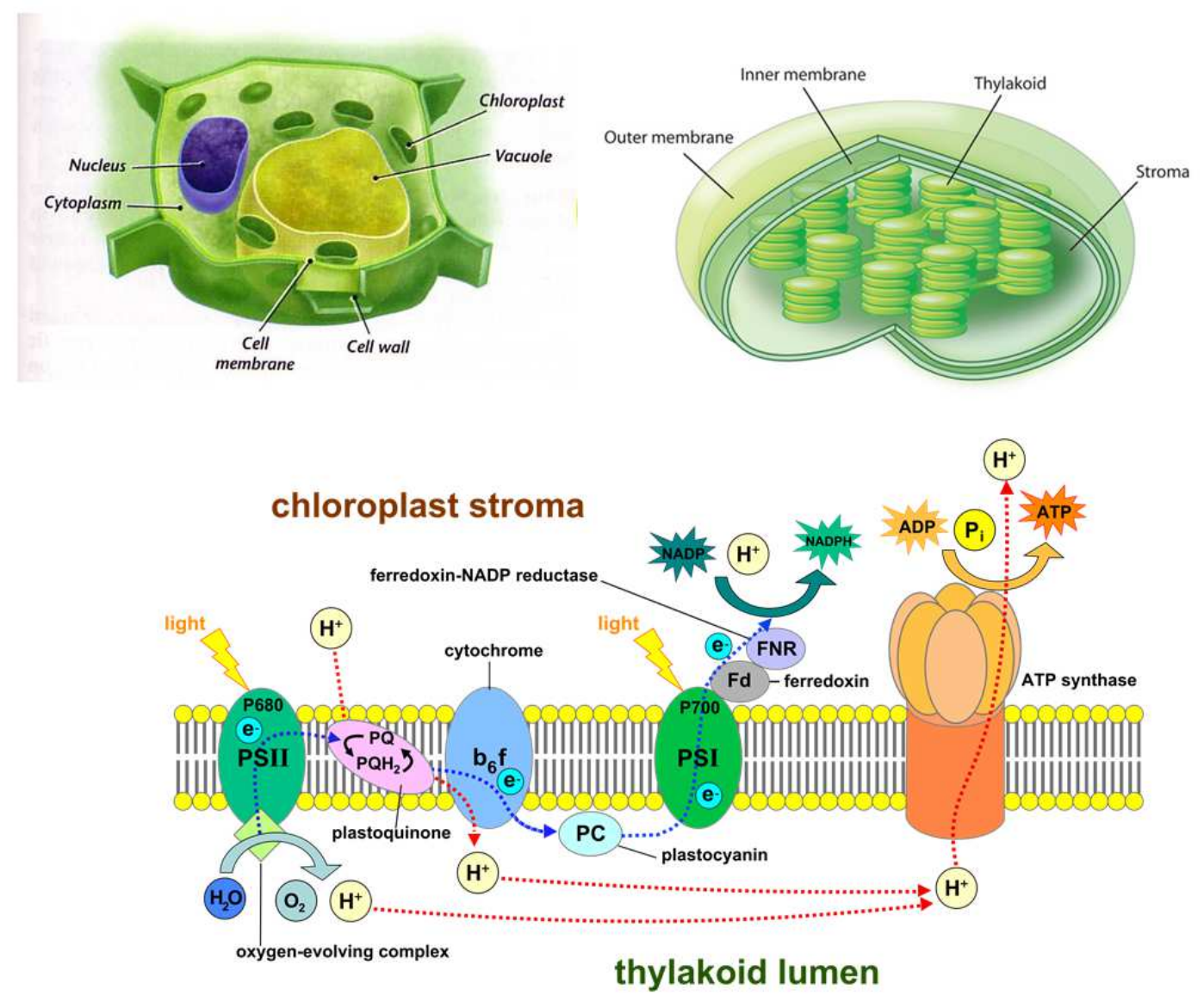

Figure 3: The location of the photosynthetic electron transport chain in plants. Top left shows a plant cell, top right shows a chloroplast. The bottom image shows the proteins responsible for light capture and photophosphorylation localized in the thylakoid membrane. [8]

Although there are many variations in such complex biochemical systems, the photosynthetic electron transport chain primarily uses the pathway illustrated in Figure 3. Starting at photosystem II, light excites electrons in the light harvest complexes adjacent to Photosystem II, causing water to be hydrolyzed in a process known as oxygen evolution. [9] The two electrons 
stripped from water are then received by plastoquinone forming plastoquinol, a lipid bound redox molecule that shuttles electrons from PSII to cytochrome $b_{6} f$. Cytochrome $b_{6} f$ then pushes four protons across the membrane and delivers the electrons to plastocyanin, a water soluble electron shuttle. Plastocyanin reduces Photosystem I which then after accepting energy from photons, excites the electrons once again and reduces an iron-sulfur complex bound to PSI. These electrons can then be used by water soluble proteins such as ferrodoxin to reduce NADP + to NADPH, another key metabolite in plant biochemistry.
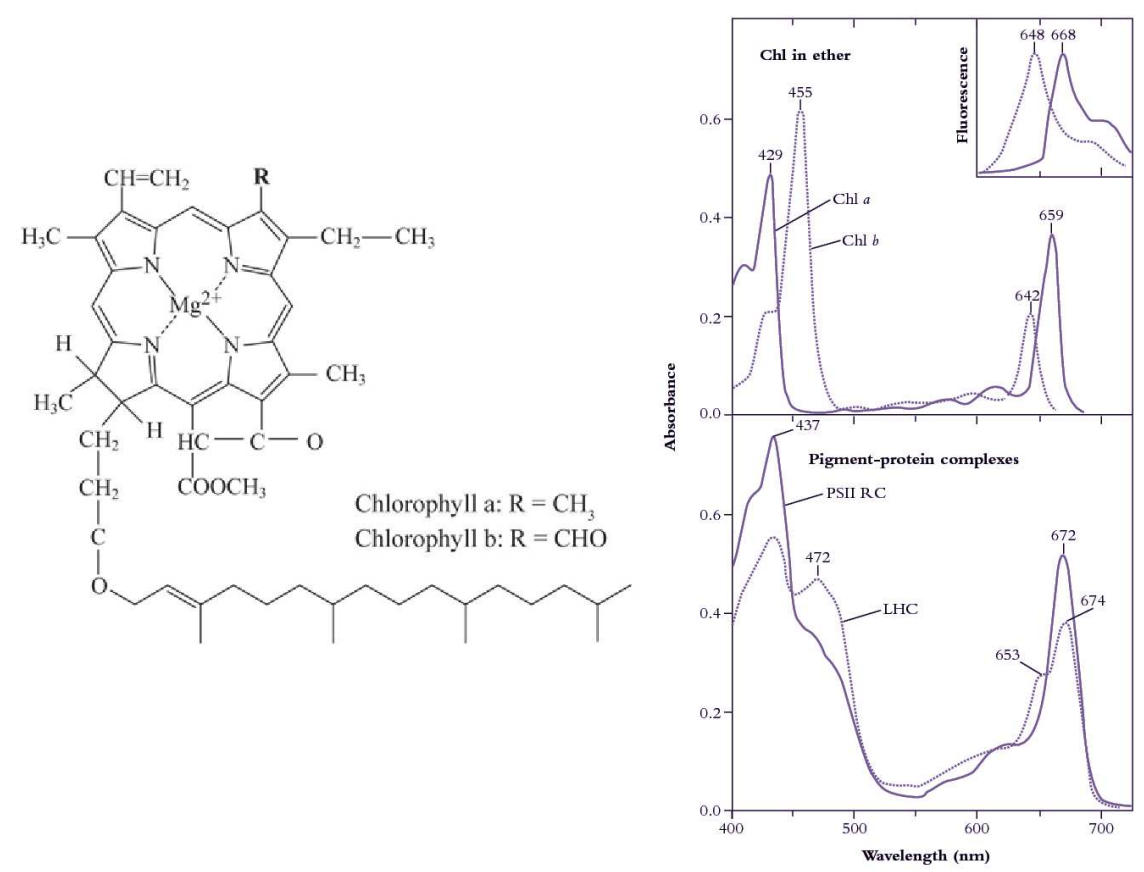

Figure 4: The molecular structure of chlorophyll a \& b as well as the action spectra for chlorophyll, photosystem II and light harvesting complexes.[10]

Chlorophyll is the primary pigment used in plants to capture photons for photosynthesis. Chlorophyll is characterized as having a porphyrin ring surrounding a magnesium ion and a phytol tail.[10] The two most common types of chlorophyll are chlorophyll a \& b, which due to small changes in their structure have differing wavelengths at which they will absorb light, as seen in Figure 4. Chlorophyll a is present in both Photosystem I and II as well as in the light harvesting complexes (LHC) that surround the photosystems. Chlorophyll b is present in PSI and LHCs but not in the PSII core complex, meaning it is possible to identify spectroscopically which proteins are present in a given solution by looking at the ratios of chlorophyll a/b. 


\subsubsection{Photosystem II}
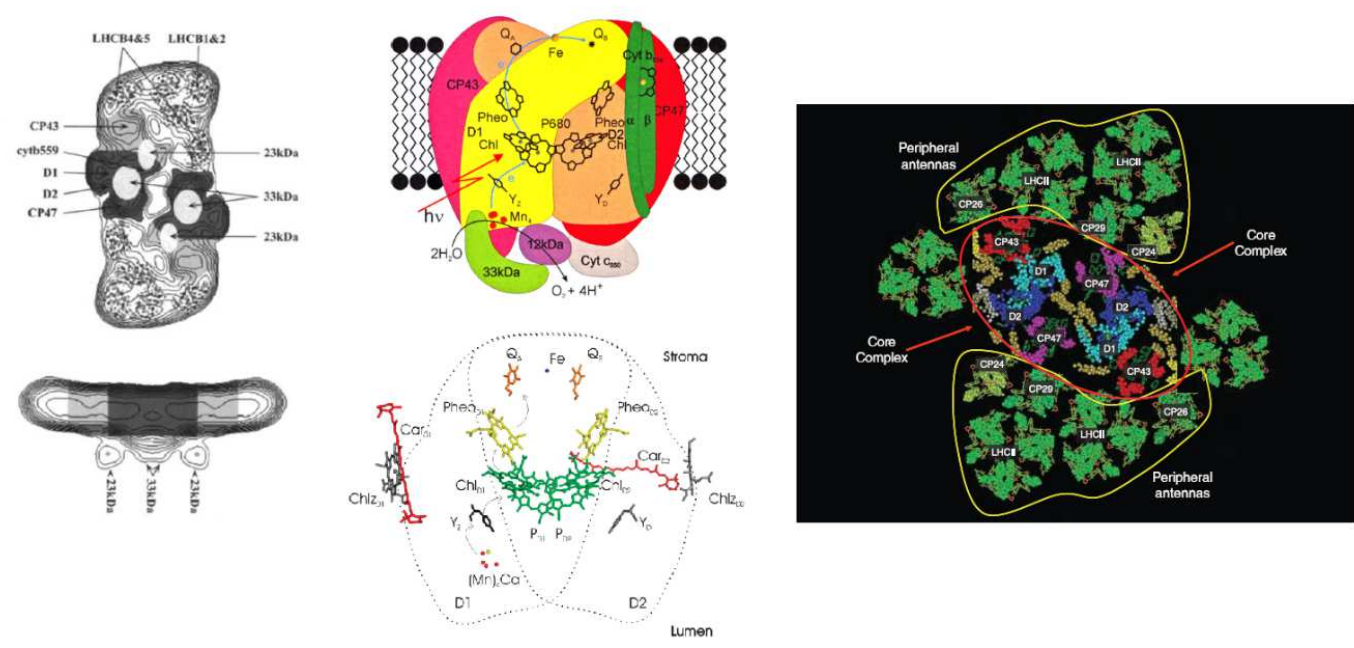

Figure 5: The structure of the Photosystem II protein complex. Left, electron microscopy images of PSII cores showing the organization of key proteins in the complex as well as how the water oxidizing subunits protrude out of the thyalkoid membrane.[11] Center, schematic of electron flow during oxygen evolution in the entire PSII core and the specific chromaphores and redox moelcules involved.[12] Right, computer generated image of a possible PSII and LHC complex arrangement in the thylakoid membrane.[13]

Photosystem II, pictured in Figure 5, is the first photosynthetic protein in the electron transport train of the thylakoid membrane and is responsible for hydrolysis of water. This splitting of water into protons and oxygen is referred to as oxygen evolution. Photosystem II is not a single protein but a collection of over 20 different transmembrane protein subunits that come together to form a single active photosynthetic unit. Both PSI and PSII do not normally absorb photons directly, but instead rely on adjacent LHCs in the thylakoid membrane to capture photons and deliver their energy into their respective photosystem by a process known as electronic energy transfer (also known as Förster Resonance Energy Transfer or FRET) whereby the electrons hop between chlorophyll molecules within the proteins.[14] At the center of photosystem II sits a collection of specialized pigments and cofactors that are responsible for oxygen evolution, as seen in figure 5. The process of oxygen evolution begins when Chlorophyll D1 is excited and transfers an electron to Pheophytin (Pheo), a chlorophyll molecule that is missing its magnesium ion. Pheo reduces Qa, a plastoquinone molecule inside of PSII, which in turn reduces Qb, which finally reduces plastoquinone molecules in the lipid membrane. The electron hole in Chlorophyll D1 is filled 
when P680 (a dimer of chlorophyll a molecules) is excited and donates an electron. This oxidized form of P680 (P680+) is the strongest oxidizing agent known in biology. The amino acid residue $\mathrm{Yz}$ (tyrosine) then fills the electron hole in $\mathrm{P} 680+$ forming $\mathrm{Yz}+\mathrm{Yz}+$ then regains its electron by oxidizing the oxygen evolving complex (OEC). The OEC is a cluster of magnesium, calcium and oxygen, which when oxidized will strip electrons from free water producing protons and oxygen. The mechanism by which the OEC produces diatomic oxygen is still not completely understood.

\subsubsection{Cytochrome $\mathrm{C}$}
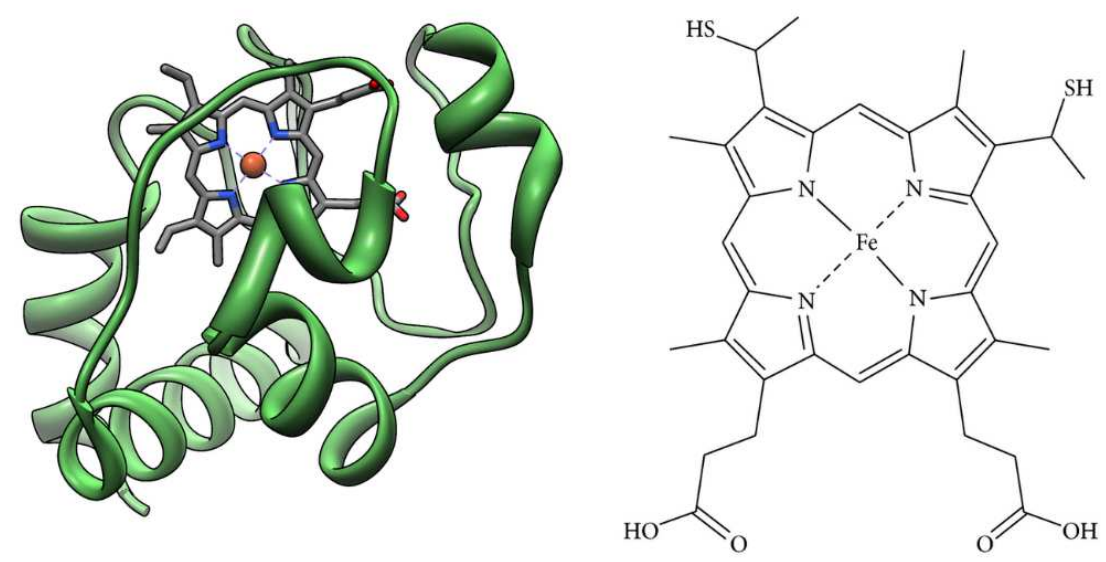

Figure 6: 3D structure of Cytochrome C (left) and the molecular structure of heme (right).[15]

Cytochrome C, pictured in Figure 6 is a small, water soluble hemeprotein with a molecular weight of about $12 \mathrm{kDa}$. [16] Cytochrome $\mathrm{C}$ is commonly found in the mitochondria where it participates in the electron transport chain shuttling electrons from Complex III to Complex IV. Interestingly, cytochrome $\mathrm{C}$ will not participate in any redox reactions with oxygen. The amino acid composition of cytochrome $\mathrm{C}$ is extremely conserved between different species, however it does have different isoelectric points depending on its sources. When extracted from plants and animals the protein has an isoelectric point of around $\mathrm{pH} 10$, however cytochrome $\mathrm{C}$ extracted from fungi has been found to have an isoelectric point of around $\mathrm{pH}$ 7. [17] Since cytochrome C frequently interacts with lipid membranes and does so by binding to the headgroups of lipids, this interaction is highly dependent on the $\mathrm{pH}$ of the solution. [18] This property of cytochrome $\mathrm{C}$ is thought to be due to the basic amino acids lysine on the proteins surface. 
A little known function of cytochrome $\mathrm{C}(\mathrm{Cc})$ in plants is to provide an alternate route for electron flow in the photosynthetic electron transport chain by oxidizing the membrane electron shuttle plastoquinol (PQ). [19] Researchers have shown definitively that PQ and Cc are redox active in intact thylakoid membranes and in liposomes of varying lipid composition. Interestingly, it has been demonstrated that the highest initial rate of electron transfer was in liposomes containing a ratio of 1 : 100, PQ : lipid by mass, and that higher PQ : lipid ratios actually had lower initial electrons transfer rates. This is thought to be due to the localization of $\mathrm{PQ}$ in the liposomes, at low PQ:lipid ratios PQ is found closer to the surface of lipid membrane.[20] The rate of PQ-Cc electron transfer in low PQ liposomes corresponds well with the electrons transfer rates observed in thylakoid membranes which have even lower PQ:lipid ratios of roughly 1 : 140. Also investigated was the effects of differing lipid composition on PQ-Cc electrons transfer. Namely the researchers made liposomes which had different proportions of lipids with negatively charged headgroups. It is known that $\mathrm{Cc}$, with its positively charged surface at near neutral conditions, will electrostatically bind to lipids with negative headgroups, forming inverted micelles allowing it to interact directly with membrane bound molecules.[21] As expected, it was found that in the absence of SQDG and PG, negative headgroup lipids, electron transfer between PQ-Cc was severely inhibited and completely stopped after 2-3 minutes. Interestingly the same effect was also observed when MGDG was removed from the liposomes, this follows as MGDG favors a HII phase (inverted micelle) when alone in solution. Clearly the proportion of PQ and the composition of lipids in the membrane Cc interacts with has a substantial effect on the electron transfer kinetics.

Cytochrome $\mathrm{C}$ has been extensive studied for its electron transfer (ET) kinetics in order to better understand electrochemical pathways in biological systems. [22] Redox properties of Cc or any such protein can be determined by using a standard three electrode cyclic voltammetry setup. However, problems resulting from adsorption of $\mathrm{Cc}$ onto bare electrodes such as $\mathrm{Au}$ or $\mathrm{Ag}$ can make ET kinetics difficult to observe. Cc will spontaneously bind to most standard electrodes in an irreversible fashion, impeding its normal mobility and leading to protein unfolding and denaturation, all of which has negative effect on its native ET properties. Through careful control of the ionic strength of the solution containing the $\mathrm{Cc}$, reversible ET can be observed on bare electrodes.[23] This of course limits the conditions under which we can study ET properties of 
Cc. This is especially troublesome if one was to attempt to use Cc as part of an electrochemical system where Cc was being used to rapidly transfer electrons from redox molecules in a solution to an electrode. One solution to this is to functionalize the surface of the working electrode in order to avoid or mitigate problems caused by adsorption. The standard approach is to use an Au electrode and functionalize the surface with a thiolated molecule. Thiols spontaneously bond to gold surfaces allowing us to create self-assembled monolayers (SAM) with whatever kind of surface properties we desire, as pictured in Figure 7.[24] The most promising of these SAMs are those of w-hydroxyalkanethiols, such as 2-mercaptoethanol. Electrode surfaces with hydroxyl terminated SAMs are found to completely eliminate any adsorption of Cc onto their surface. This lack of adsorption also leads to greater ET kinetics compared to other SAMs. Another common SAM used in Cc redox studies are carboxyl terminated SAMs. These surfaces strongly adsorb Cc as the overall charge of $\mathrm{Cc}$ is positive at near neutral $\mathrm{pH}$ conditions, allowing the carboxylic acid group to capture Cc with a powerful electrostatic bond. This binding does not allow Cc to orient its heme group into an optimal position for ET, leading to poor electron coupling.[25] The result is that hydroxyl terminated SAMs have electron transfer rates that are about two orders of magnitude higher than that of carboxyl terminated SAMs.

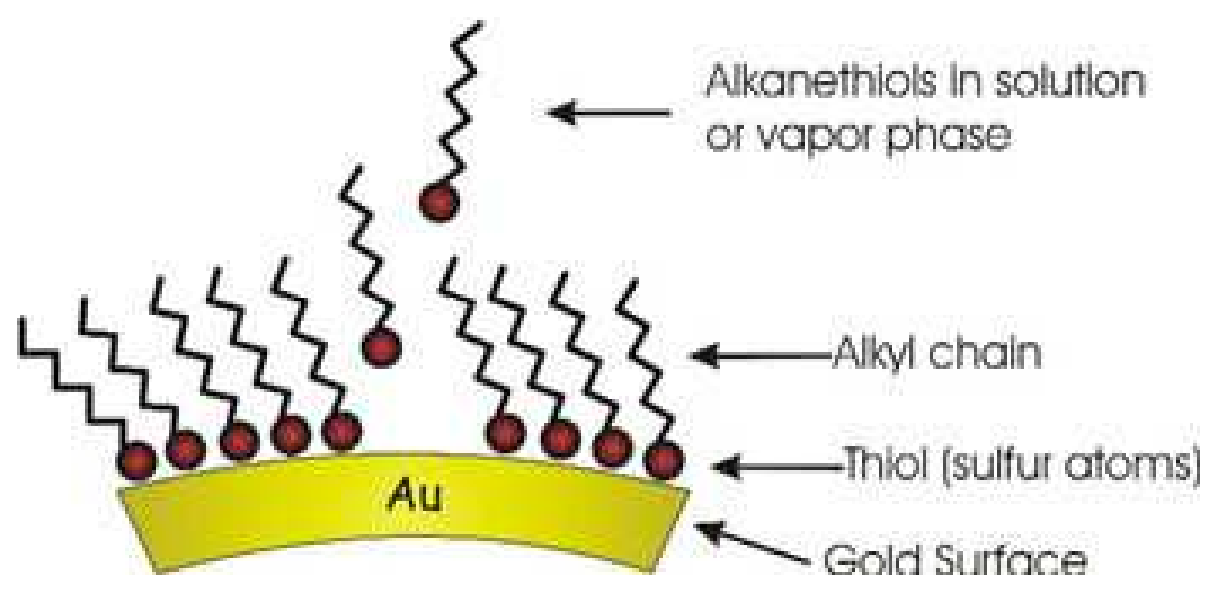

Figure 7: Diagram of an Au self-assembled monolayer showing the orientation of thiols and alkyl on the gold surface.[26] 


\subsubsection{Thylakoid Membrane and Lipid Dynamics}

The thylakoid is a self contained lipid membrane localized in the chloroplast of plants. It houses the photosynthetic electron transport chain of plants, and is responsible for converting solar energy into usable forms of chemical energy. By dry mass about half of the thylakoid membrane is proteins and the other half is lipids and lipid soluble biomolecules such as plastoquinol, $\alpha$-tocopherol or $\beta$-carotene. These lipids are essential for the proper functioning of photosynthetic proteins, as they participate in the assembly and regulation of these large multimeric protein complexes. [27]
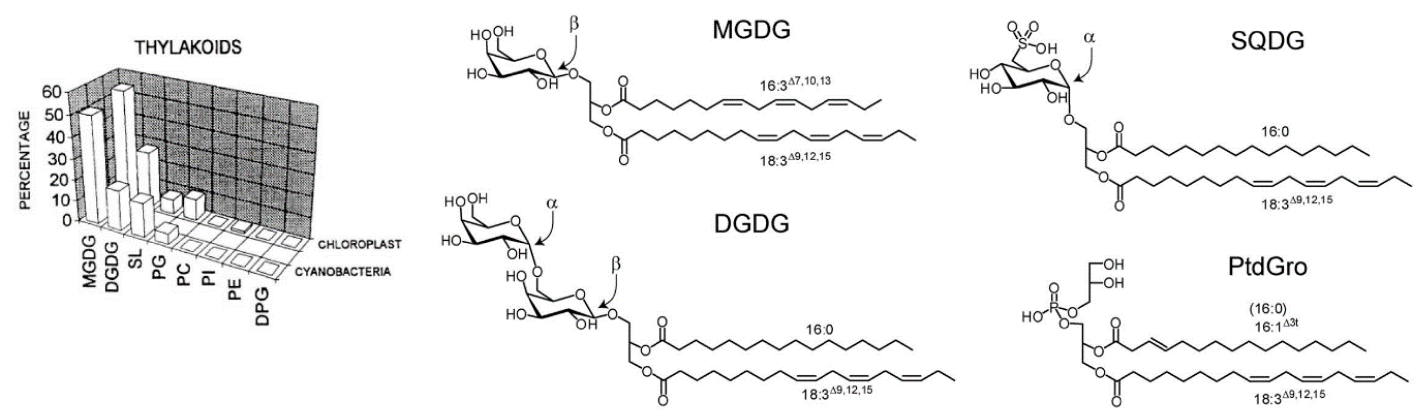

Figure 8: Breakdown of lipid proportions in chloroplast and cyanobacterial thylakoid membranes and the molecular structure of key thylakoid lipids, monogalactosyldiacylglycerol (MGDG), digalactosyldiacylglycerol (DGDG), Sulfoquinovosyldiacylglycerols (SQDG), Phosphatidylglycerol (PG), Phosphatidylcholine (PC), Phosphatidylinositol (PI), Phosphatidylethanolamine (PE), and Diphosphatidylglycerol (DPG). [27]

Lipids are amphiphilic, meaning they have a hydrophilic and hydrophobic region. If we take MGDG as an example, the hydrophilic section is the galactose sugar ring, this section is referred to as the lipid headgroup. The hydrophobic region constitutes the two acyl chains, which are connected by a glycerol molecule. When the lipids found in a thylakoid are mixed together they form a lipid bilayer. This membrane is the barrier that separate cells from their surroundings and is found in all living organisms. However, the formation of a lamellar phase or lipid bilayer is dependent on the type of lipids comprising the membrane and the proportions of these lipids. The main lipid constituents of thylakoid membranes are MGDG, DGDG, SQDG and PG. As seen in Figure 8, plant inthylakoid membranes they are found in mole \% of roughly $50 \%$ MGDG, $25 \%$ DGDG, $20 \%$ SQDG / PG, with the remaining $5 \%$ consisting of various other phospholipids. Taken together these lipids will form a bilayer, however isolated lipids can form different lipid phases.[28] 
Figure 9 shows how lipids can adopt different configurations depending on their molecular properties and proportions relative to other lipids. For example MGDG when isolated will spontaneously form an HII lipid phase seen in Figure 9, where the headgroups of the lipids group together and the acyl chains face outward into the solution. The HII phase is also called a reverse micelle, where a micelle is a HI phase where the lipid headgroups face outwards to the solution and the acyl chains group together but do not form a bilayer. The reason MGDG takes this HII phase is because of its shape within a lipid membrane, the desaturation of both acyl chains causes its hydrophobic region to flare out, while its headgroup is comparatively narrow. This causes MGDG to have a triangular shape, as seen in Figure 9. Another important aspect of lipids is the electrostatics of its headgroup. The headgroups of MDGD and DGDG are sugars or saccharides, and are therefore neutral. SQDG and PG on the other hand are negatively charged at neutral $\mathrm{pH}$, and are therefore able to bind positively charged ions and molecules while repelling negatively charged ones. This has important implications for how these membranes organize themselves and what types of molecules they will interact with.
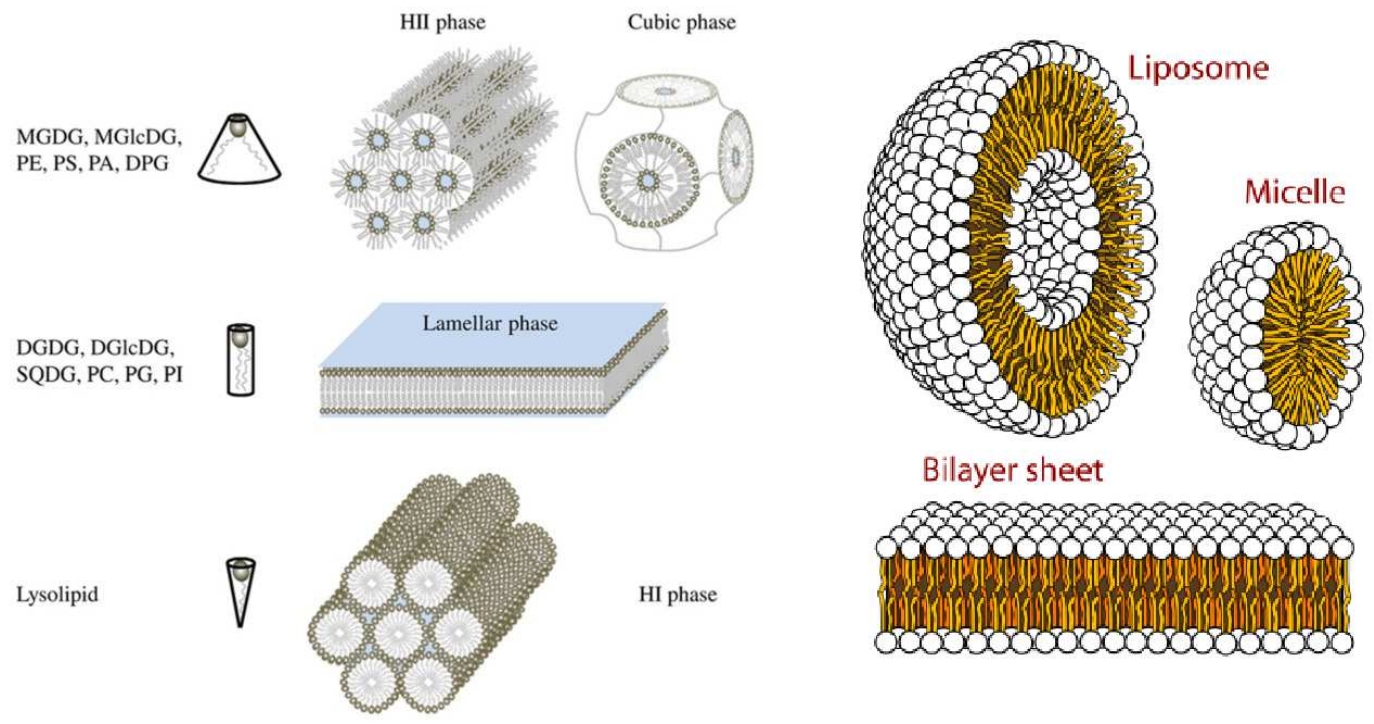

Figure 9: Common phase structures of thylakoid lipids (left). Possible bilayer structures for lamellar and HI phase lipids (right). [28] 


\subsection{Chromatography}

Chromatography refers to any technique that separates a mixture of compounds by exploiting the difference in mobility of a given molecule, based on its affinity to a mobile phase and stationary phase of a given material. [29] This is illustrated in Figure 10, where the stationary phase contains solute particles that are bound to the chromatographic material, while the mobile phase is a solvent that contains solute particles that are not bound and can more easily travel past or through the chromatographic material. Exploiting the differences of a given analytes affinity to the stationary or mobile phase means that different compounds will exit or elute out of the chromatographic material at different rates, allowing us to isolate them. In the case of this body of work, the stationary phase is a silica matrix, the most common adsorbent used in chromatography. Silica is used as it is relatively inert allowing for reusability and can be made into microporous beads allowing for an increased surface area for adsorption.

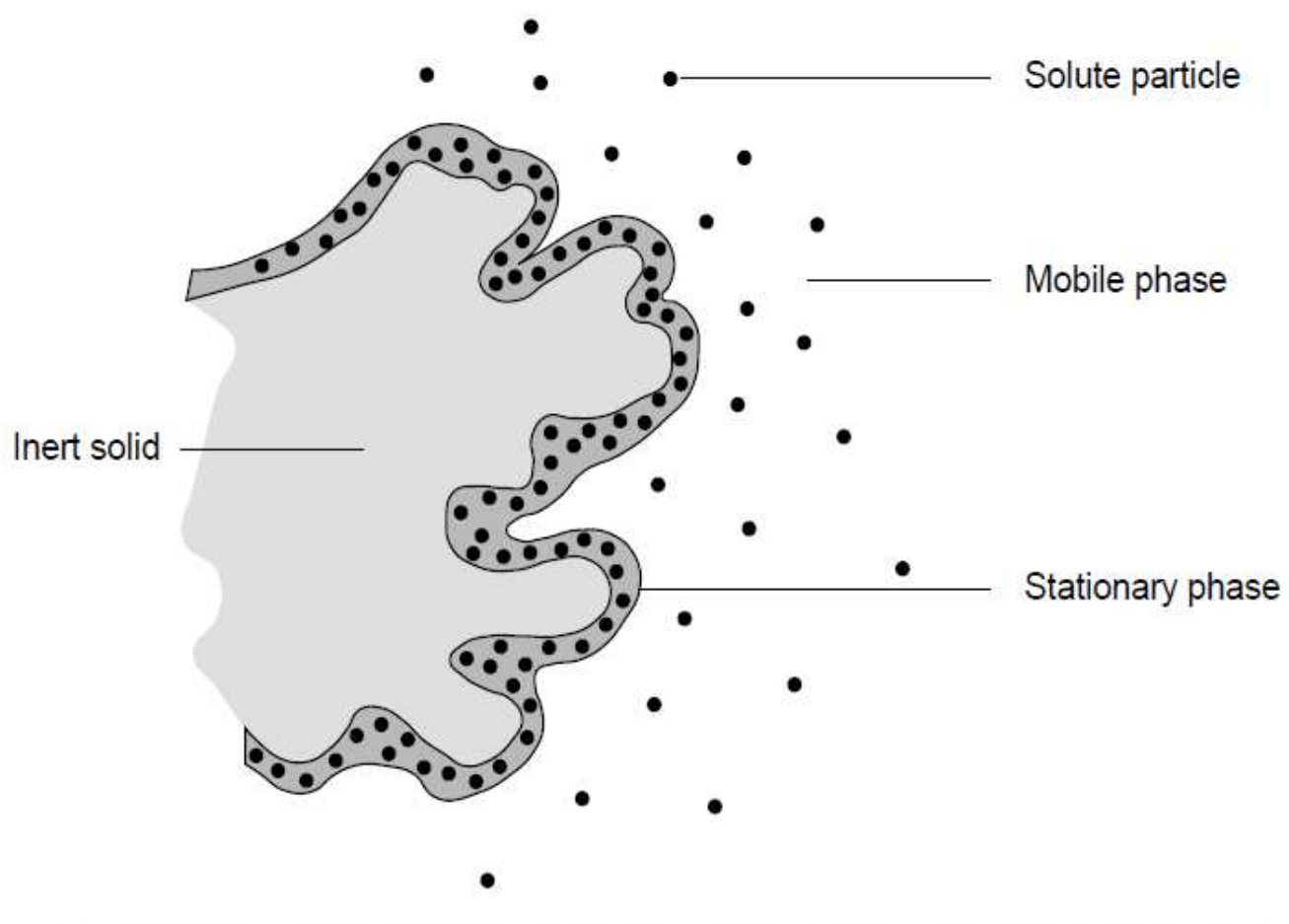

Each solute partitions itself between the stationary phase and the mobile phase

Figure 10: Diagram of solute particles separating between the mobile and stationary phases around a chromatographic material. [29] 
Column chromatography and thin layer chromatography are the most common methods by which silica matrices are used for chromatographic separation. Column chromatography uses a long column packed with silica beads, where the compounds of interest are 'loaded' at the top of the column. As the solvent (mobile phase) is run down the column, the potential analytes loaded at the top also travel down the column, at a rate determined by their affinity for the mobile or stationary phase. Molecules that are strongly adsorbed to the silica travel slowly or can even remain at the top of the column while molecules that adsorb weakly travel faster. Modern column chromatography relies on pressurized columns that quickly force a solvent through the silica matrix, this is known as a 'flash column'. [30] This technique is extremely powerful as it is many times faster than gravity column chromatography but still has very high resolution provided that the column is packed with higher grade silica with smaller mesh or particle size.

Thin layer chromatography (TLC) functions in a very similar fashion. A TLC plate is usually a glass substrate with a thin layer of silica gel adhered to its surface. The analyte mixture is loaded onto the plate by first dissolving it in an appropriate solution and 'spotting' it onto the plate using a capillary tube. The solvent is evaporated away and the analyte mixture is now bound to the silica. When the plate is placed in a solution of solvent, as in Figure 11, the solvent moves up the plate and will in turn carry the loaded analytes along with it. Just as in column chromatography, the affinity of an analyte to the mobile and stationary phase will determine the distance it travels up the plate.

Analytes on a TLC plate can be visualized or identified using a number of different techniques. If a compound is UV active, for example having multiple conjugated carbons adjacent to each other, an ultraviolet lamp can reveal its location on the plate. Some TLC plates even have a fluorescent indicator embedded in the silica, which when illuminated with an appropriate wavelength of light the entire plate will glow a color (usually green) and the area containing the compound of interest will appear as a dark spot or shadow against the background. Other visualization techniques involve applying a reagent to the TLC plate known to react with one or more of the functional groups on the compound of interest. These techniques can be very specific, such as Dragendorff's reagent which will only react with nitrogen containing compounds. Other 


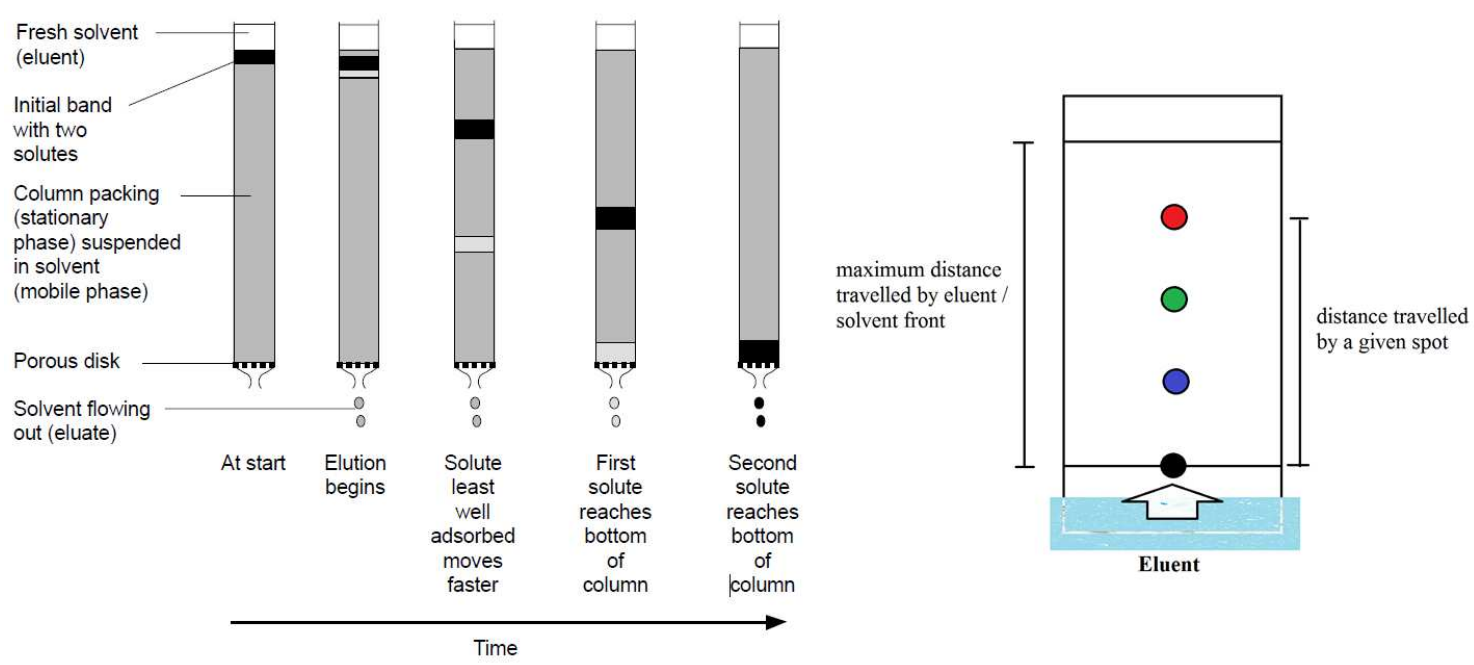

Figure 11: Diagram of column chromatography (left) and thin layer chromatography (right) in process.

$[29]$

techniques are non-specific, for example dilute sulfuric acid spray followed by heating will reveal any compounds on the plate that have hydrocarbons present. Some techniques are non-destructive and can be used to reveal a compound location without altering it significantly, allowing it to be removed from the TLC plate for further analysis. These include the use of UV lamps or exposing the TLC to iodine which reversibly binds many functional groups present in organic molecules.

\subsection{Electrochemistry}

\subsubsection{Oxygen Reduction Reaction}

The oxygen reduction reaction (ORR) is the reaction by which oxygen is reduced to water. [31] This reaction is the basis for hydrogen and methanol fuel cells, taking place at the cathode. The kinetics of this reaction are normally extremely slow, and therefore require a catalyst in order to perform on a practical level. The most common catalyst used is platinum and while it is very effective at catalyzing ORR, it is extremely expensive. Most of the research regarding ORR and fuel cells seeks to minimize the usage of platinum or to find cheaper alternatives. Pictured in Figure 12 is the standard 'dissociative' ORR mechanism in acidic conditions.

The reaction proceeds by first adsorbing oxygen onto its surface, then splitting or disso- 

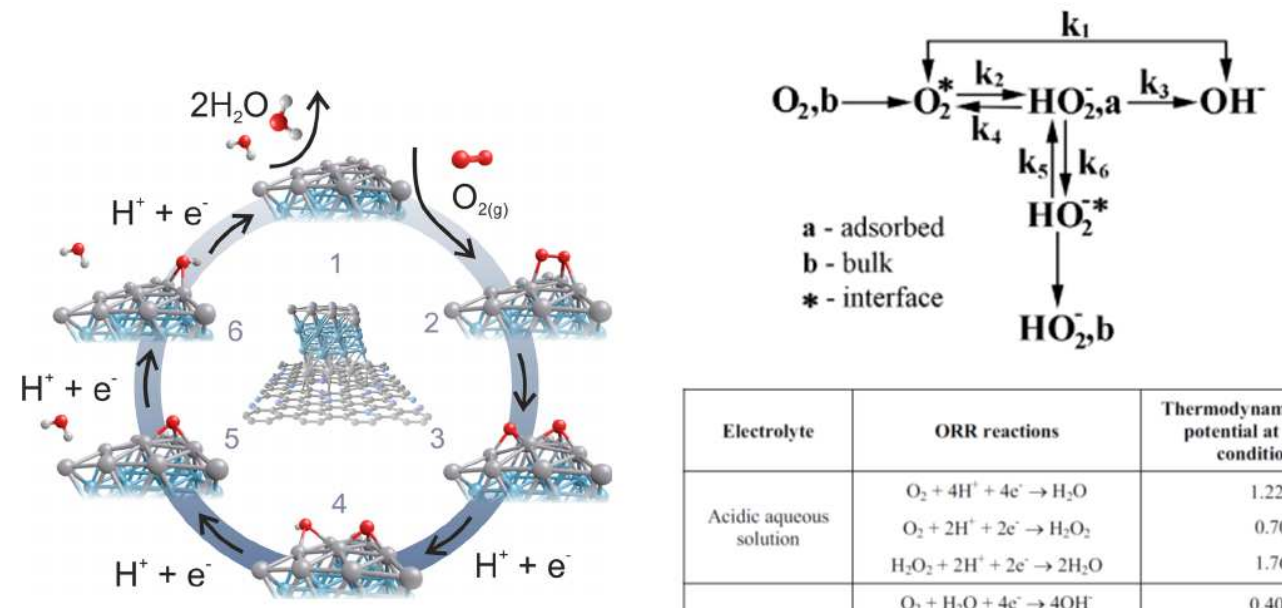

\begin{tabular}{|c|c|c|}
\hline Electrolyte & ORR reactions & $\begin{array}{c}\text { Thermodynamic electrode } \\
\text { potential at standard } \\
\text { conditions, } \mathbf{V}\end{array}$ \\
\hline \multirow{2}{*}{$\begin{array}{c}\text { Acidic aqueous } \\
\text { solution }\end{array}$} & $\mathrm{O}_{2}+4 \mathrm{H}^{+}+4 \mathrm{e}^{-} \rightarrow \mathrm{H}_{2} \mathrm{O}$ & 1.229 \\
& $\mathrm{O}_{2}+2 \mathrm{H}^{+}+2 \mathrm{e}^{-} \rightarrow \mathrm{H}_{2} \mathrm{O}_{2}$ & 0.70 \\
$\mathrm{H}_{2} \mathrm{O}_{2}+2 \mathrm{H}^{+}+2 \mathrm{e}^{-} \rightarrow 2 \mathrm{H}_{2} \mathrm{O}$ & 1.76 \\
\hline \multirow{2}{*}{$\begin{array}{c}\text { Alkaline aqueous } \\
\text { solution }\end{array}$} & $\mathrm{O}_{2}+\mathrm{H}_{2} \mathrm{O}+4 \mathrm{e}^{-} \rightarrow 4 \mathrm{OH}$ & 0.401 \\
& $\mathrm{O}_{2}+\mathrm{H}_{2} \mathrm{O}+2 \mathrm{e}^{-} \rightarrow \mathrm{HO}_{2}^{-}+\mathrm{OH}$ & -0.065 \\
& $\mathrm{HO}_{2}^{-}+\mathrm{H}_{2} \mathrm{O}+2 \mathrm{e}^{-} \rightarrow 3 \mathrm{OH}$ & 0.867 \\
\hline
\end{tabular}

Figure 12: Mechanism of oxygen reduction reaction in acidic environment (left).[32] Dissociative and associative reaction pathways for basic \& neutral oxygen reduction reaction (top-right).[31] Breakdown of standard electrode potentials for all acidic and basic oxygen reduction reactions (bottom-right).[31]

ciating diatomic oxygen into two separate oxygen atoms. If this dissociation does not occur the reaction is considered 'associative' and the end product will be hydrogen peroxide instead of water. This reaction has a lower thermodynamic electrode potential and can lead to electrode fouling. Avoiding this reaction is one of the principle reasons $\mathrm{Pt}$ is the catalyst of choice in ORR as it readily dissociates diatomic oxygen. After oxygen is dissociated, the electrons from the electrode and protons from the solution react with oxygen, first to form $\mathrm{OH}$ or hydroxyl groups. This reaction is then repeated, resulting in water as the final product. Also pictured in Figure 12 is the ORR taking place under basic or neutral $\mathrm{pH}$ conditions. The mechanism is the same as in acidic conditions, except that the source of protons is water and the final product is $\mathrm{OH}$ or hydroxyl groups. This high $\mathrm{pH}$ reaction also has an associative and dissociative pathway, and platinum again favors the dissociative pathway. This high $\mathrm{pH}$ reaction does however have a substantially lower thermodynamic electrode potential.

\subsubsection{Cyclic Voltammetry}

Cyclic voltammetry (CV) is a standard technique used to investigate electrochemical systems. [33] As seen in Figure 13, the principle is to apply a voltage to an electrode of interest and measure 
the current, that is consumed or produced as the voltage is cycled forwards or backwards. This is done using a three electrode system, where the working electrode is under investigation, a counter electrode is used to control and measure the current and a reference electrode is used to calibrate and control the cell voltage. In a typical CV experiment, as the voltage is increased from negative to positive, oxidation will occur and a negative current will be produced. The highest current generated on this forward sweep is the anodic peak. Then the voltage is swept back, going from positive to negative and a reduction reaction occurs. This generates a positive current as electrons are consumed, the highest current generated here is the cathodic peak. The position and strength of the anodic and cathodic peaks can be used to examine the electrochemical properties of an electrode and its related redox reactions.
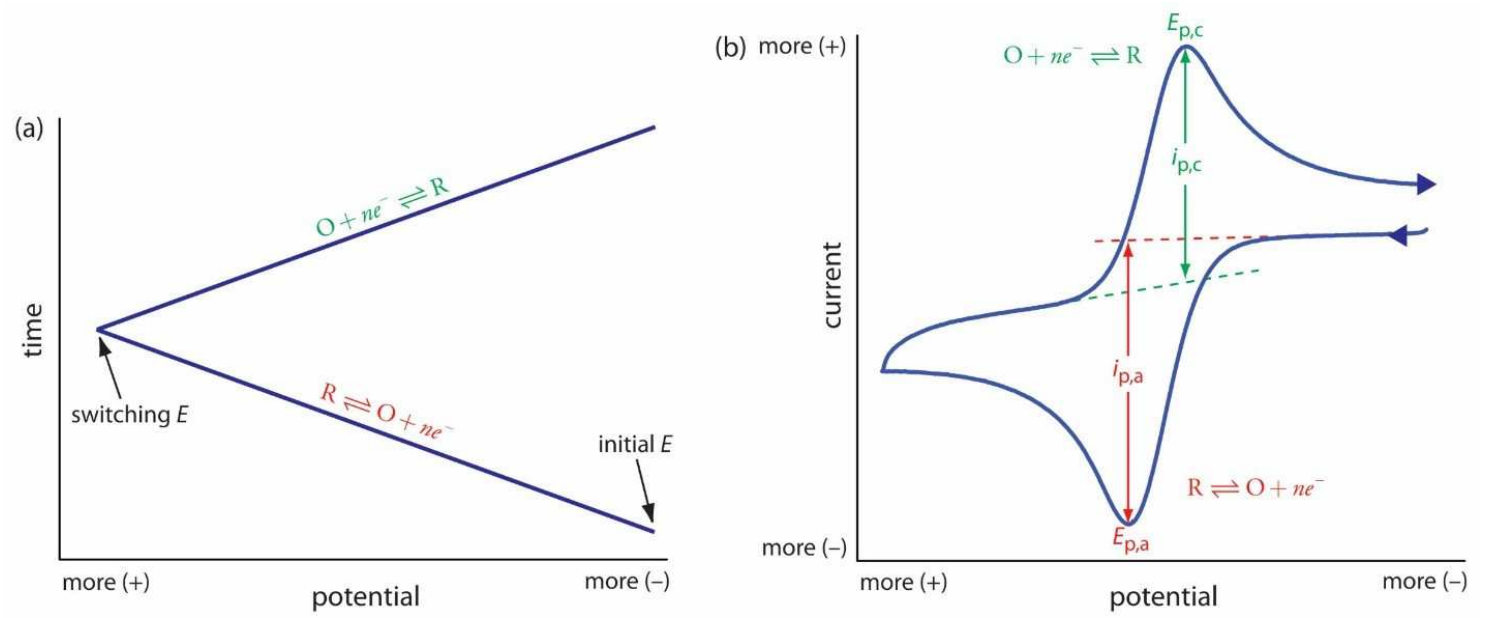

Figure 13: Basic methodology of cyclic voltammetry. On the left is the starting and ending potential over time. On the right is the change in current versus the potential as it is swept forwards and backwards.[34]

\subsection{Literature Review}

The last decade has seen a substantial rise in the amount of research that tries to integrate photosynthetic proteins into photovoltaic devices in an attempt to increase efficiency or lower the overall cost of such devices. One common idea is to embed photosystem I into a solid state device. Since photosystem I is at the end of the standard photosynthetic transport, its function is to accept low energy electrons from plastocyanin and expel excited electron after excitation via photons, making it is an obvious candidate for solid state devices. One such example published in ACS Sustainable 
Chem. Eng., involved sandwiching PSI particles between FTO/PEDOT:PSS and LiF/Al. [35] FTO/PEDOT:PSS are used as transparent conducting materials at the cathode, while LiF is used to oreint the PSI. This system is depicted in Figure 14. Using the permanent dipole of PSI at its $\mathrm{Fb}$ site (where normally electrons are expelled into iron-sulfur clusters for NADPH production), the photosystems have a semi-ordered orientation with the $\mathrm{Fb}$ side facing the $\mathrm{LiF} / \mathrm{Al}$ anode.

a)

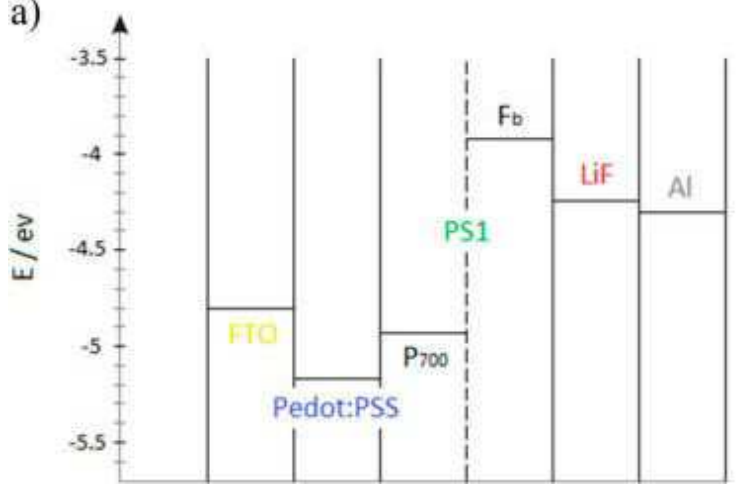

b)

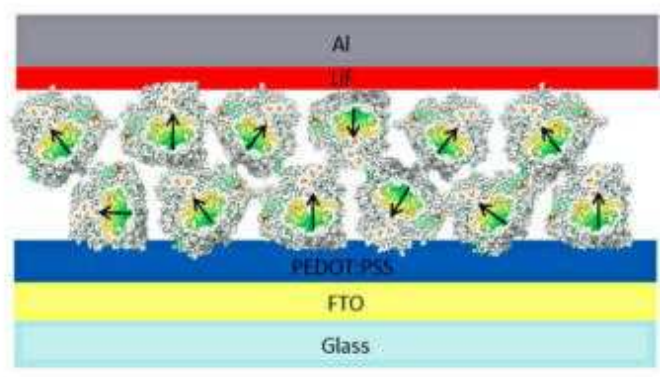

Figure 14: Energy diagram of the various components in the solid state PSI based solar cell (left). Diagram showing the arrangement of the solid state solar cell components (right). [35] Light passes through the glass, FTO and PEDOT:PSS layer and excites electrons in the PSI layer, which are then received by the $\mathrm{LiF} / \mathrm{Al}$ anode, leaving a hole in the FTO/PEDOT:PSS. This electron is run through an external circuit and recombine with the hole at the FTO/PEDOT:PSS cathode.

After illumination the excited electrons are collected by the $\mathrm{LiF} / \mathrm{Al}$ anode and flow towards the FTO cathode. P700 is the pigment complex that acts as the primary electron donor for PSI, after illumination it forms its P700+ state. P700 has a similar energy level to PEDOT:PSS, therefore electrons will be stripped from the PEDOT:PSS to reduce P700+ to its original state, while the hole left in the PEDOT:PSS migrates into the FTO to recombine with the electron. The solution of PSI used is extremely concentrated and after spin coating onto the surface the device is placed under vacuum to remove the solvent. The resulting device has a current density of 0.96 $\mathrm{mA} / \mathrm{cm}^{2}$ under AM $1.5 \mathrm{G}$ sunlight conditions. This device has the highest efficiency of any PSI based solid state device.

The following device uses PSI again, but in combination with a $\mathrm{TiO}_{2} / \mathrm{ITO}$ surface. $\mathrm{TiO}_{2}$ is capable of absorbing UV light and acting as a photocatalyst for hydrolysis, $\mathrm{TiO}_{2}$ can also be used as an anode for a dye sensitized solar cell (DSSC). [36] Since $\mathrm{TiO}_{2}$ only absorbs a small fraction of 


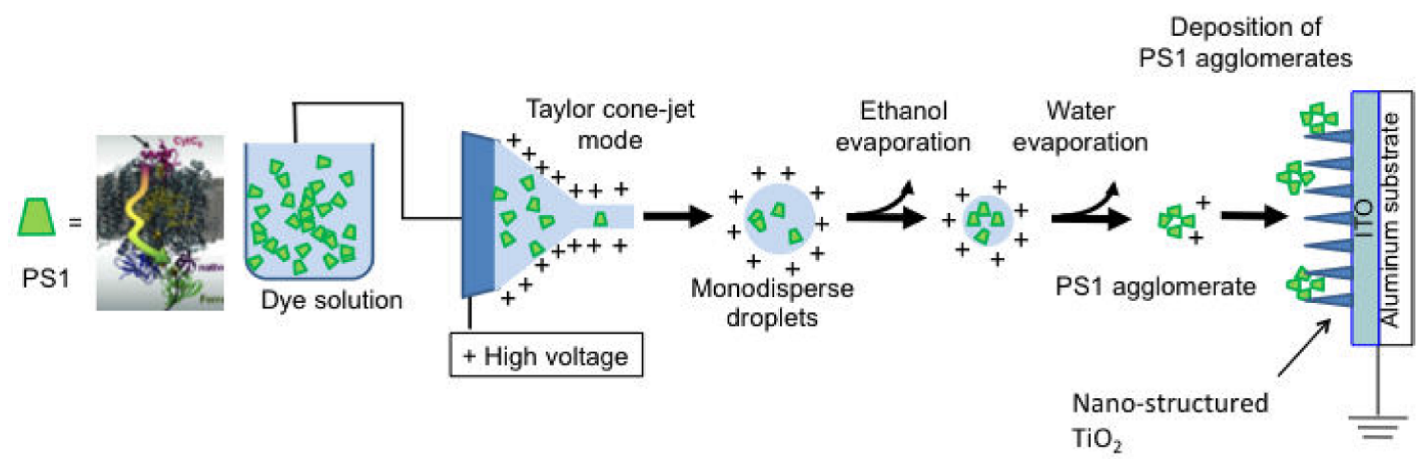

Figure 15: Diagram showing production of Photosystem I sensitized $\mathrm{TiO}_{2}$ solar cell. [37] A concentrated solution of PSI is electrosprayed onto nanostructured $\mathrm{TiO}_{2}$, evaporating its solvent in flight and aggregating on the $\mathrm{TiO}_{2}$.

the light available from the sun, a DSSC simply embeds a dye onto the $\mathrm{TiO}_{2}$ surface to increase the range of wavelengths of light that can be absorbed. Typically a DSSC is constructed using a molecular dye such as chlorophyll, however the researchers in this paper instead used the PSI protein complex as their sensitizer. Starting with a nano-column film of $\mathrm{TiO}_{2}$ on an aluminum, a solution of PSI is electrosprayed onto the $\mathrm{TiO}_{2}$. [37] During the flight of the PSI solution droplets, the solvent evaporates and only agglomerates of PSI are deposited onto the surface of the $\mathrm{TiO}_{2}$. This process is depicted in Figure 15. When combined with ITO as a substrate for the $\mathrm{TiO}_{2}$, the addition of PSI increases the current density of the device under illumination by almost four times. This however only brings the efficiency of the device up to $0.52 \%$, an extremely low efficiency compared with other DSSC devices with efficiencies of $10 \%$ or even $15 \%$ in some cases. [38]

The next device breaks from the pattern of using PSI as the primary method of photon capture and instead turns to Photosystem II. Since PSII harvests light in order to perform hydrolysis, it can both provide electrons for a devices anode while simultaneously providing the reactants for an oxygen reduction reaction at a cathode. The central notion of this device is to immobilize PSII onto an anode where upon illumination it provides electrons for the anode while the oxygen and protons produced are reduced at a cathode to reform water. [39] The photoanode is constructed using an Au electrode with a polymer of mercapto-p-benzoquinone attached to its surface. The thiol group is bonded to the Au surface while the quinone groups provide both a site of attachment for PSII proteins and an acceptor for electrons stripped from water during 


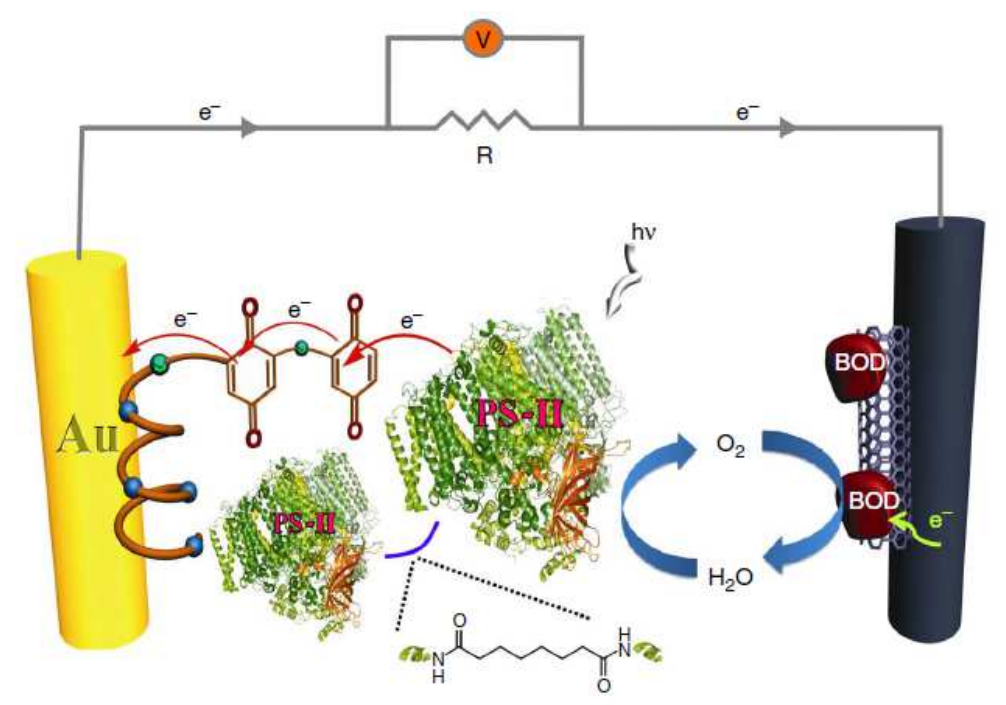

Figure 16: Diagram of a Photosystem II based photoelectrochemical cell showing anode and cathode surfaces as well as electron flow. [39] PSII is crosslinked using bis(sulfosiccinimidyl) suberate, and then bound to a $\mathrm{Au}$ anode using a polyer of mercapto-p-benzoquinone. This polymer also facilitates electron transfer from PSII to the anode upon illumination. The oxygen and protons produce by PSII oxygen evolution are reacted at a glassy carbon electrode containing single walled carbon nanotubes with bilirubin oxidase (BOD) embedded on the nanotubes. BOD uses the electrons suppilied by the gold anode to reduce oxygen and protons back to water.

hydrolysis. After addition of a PSII solution onto the anode surface, the proteins are crosslinked to each other using bis(sulfosiccinimidyl)suberate in order to form a integrated electrode. The cathode consists of a glassy carbon electrode with single walled carbon nanotubes (SWCNT) dispersed on its surface. After the addition of SWCNTs a solution of bilirubin oxidase (BOD) was deposited onto the electrode surface. This device is depicted in Figure 16. BOD is an enzyme that catalyzes the oxygen reduction reaction and will spontaneously bind to SWCNTs providing a non-platinum based cathode that will catalyze oxygen reduction. At maximum power this device has a maximum current density of $60 \mu \mathrm{A} / \mathrm{cm}^{2}$, which is extremely low.

The final device to be examined is one that uses both PSI and PSII to capture photons. However, instead of isolating and immobilizing the individual enzymes, the researchers used intact thylakoid membranes which house the photosynthetic electron transport chain. This device uses glassy carbon electrodes with a multi-walled carbon nanotube dispersion on their surface, for both the anode and the cathode. [40] 


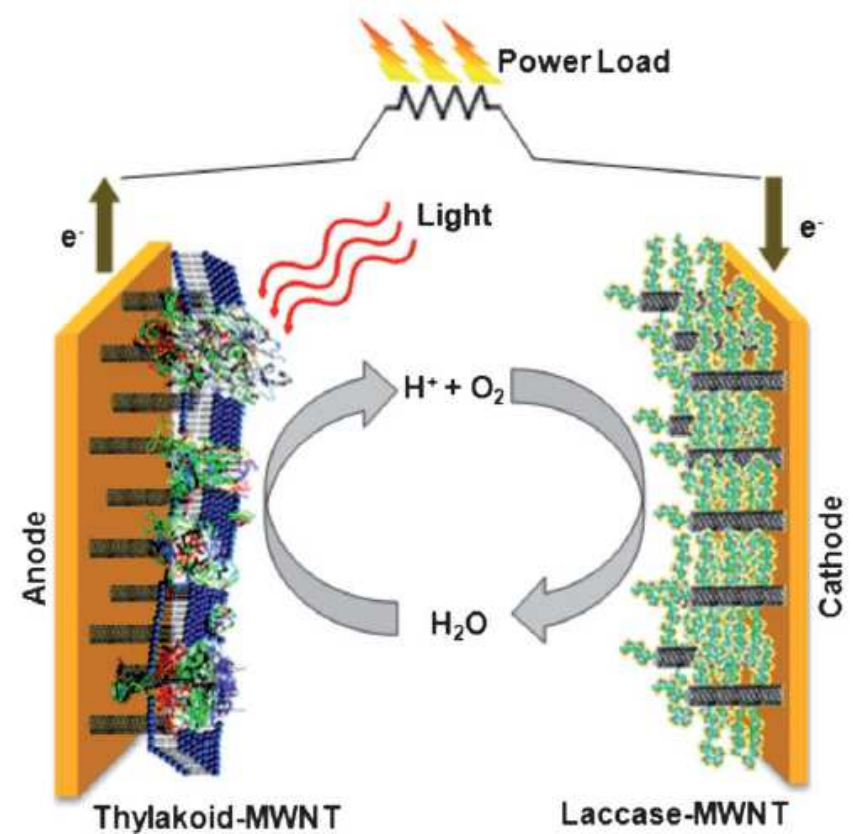

Figure 17: Diagram of a thylakoid based photoelectrochemical cell showing anode and cathode surfaces as well as oxygen evolution and reduction. [40] The anode consists of thyalkoid membranes bound to a multi walled carbon nanotube (MWNT) matrices using 1-Pyrenebutanoic acid succinimidyl ester (PBSE). The cathode consists of the same MWNT/PBSE substrate with the protein laccase bound to its surface. Laccase can catalyze oxygen reduction. When the system is illuminated with light the PSII produced oxygen and protons, the electrons stripped from water travel through an external circuit to reach the cathode where an oxygen reduction reaction reforms water. 
As with the previous device the photosynthetic proteins are embedded onto the anode, this is done using the molecule 1-Pyrenebutanoic acid succinimidyl ester (PBSE). The pyrene group binds via $\pi-\pi$ interactions to the MWNT and the succinimiydyl ester covalently binds to proteins in the thylakoid membrane. This device is pictured in Figure 17. Upon illumination, electrons have a few different paths they can take to reach the anode. Cytochrome $b_{6} f$ and plastocyanin can both directly transfer electron to the MWNT, both of which can also reduce the in solution mediators such as $\mathrm{Fe}(\mathrm{CN})_{6}$, which can subsequently deliver electrons to the MWNTs. The protons and oxygen produced by the PSII in the thylakoid membrane are then reduced at the cathode by laccase. This enzyme catalyzes the oxygen reduction reaction and is also bound to the cathode using the same PBSE-MWNT matrix. This device yields a maximum current density of $68 \mu \mathrm{A} / \mathrm{cm}^{2}$, which is high for such a device but is functionally extremely low in comparison to other solar energy conversion systems.

All of these devices share one key characteristic, they all rely on permanent immobilization of photosynthetic apparatus onto an electrode. While this does avoid problems relating to diffusion of proteins and redox molecules in a solution, putting proteins as large and complex as PSI and PSII into non-native environments means that they are prone to denaturing much quicker than they would naturally. Likewise, these proteins even in their native environments can only capture so many photons before some form of photodecomposition takes place and the proteins stop functioning as normal. In plant and cyanobacterial cells these proteins are capable of being repaired and reused however the mechanisms by which this takes place are complex and could not easy be adapted into devices such as these. [41] This presents a fundamental problem in any device attempting to utilize photosynthetic proteins for solar energy conversion.

The main goal of this body of work is to present and test an alternative to conventional methods of integrating photosynthetic apparatus into solar cell devices. Essentially the photosynthetic electron transport chain is kept at a distance from the electrodes by residing in a solution that closely resembles their native environment, while maintaining an indirect electrical connection to the electrodes using soluble redox proteins. 


\subsection{Photoelectrochemical Cell Design}

The notion of using PSII as method of catalyzing hydrolysis has already been attempted by many researchers, however the protein or photosynthetic apparatus, in the case of using a whole thylakoid membrane, is always immobilized onto anode in order to extract the electron stripped from water. Since it is now known that cytochrome $\mathrm{C}$ can readily accept electrons from plastoquinol, the primary electron acceptor for PSII, and the cytochrome $\mathrm{C}$ can also deliver electrons to an electrode, therefore it is conceivable to construct a photoelectrochemical cell (PEC) that exploits this activity to extract electrons from the photosystem to complete a circuit. The basic design of such a device is pictured in Figure 18; starting with PSII where water is split into protons and oxygen and the two electrons stripped from water are received by plastoquinone forming plastoquinol. Next, plastoquinol reduces cytochrome $\mathrm{C}$ which in turn delivers electrons to an anode. These electrons are used in an oxygen reduction reaction at a cathode where the products of photosynthetic oxygen evolution, protons and oxygen, are reduced to reform water. The cycle can then begin again.

Implementation of such a system presents a number of practical difficulties. If we want to house PSII in its native environment it will need to be surrounded by lipids. A simple solution is to embed PSII into liposomes. Pictured in Figure 9, liposomes are small often spherical lipid bilayers that emulate the properties of a cell membrane and are commonly used to study the properties of transmembrane proteins or lipid bound biomolecules. [42] A larger problem is the fact that biological system are highly sensitive to pH. PSII is most active in slightly acidic solution of $\mathrm{pH}$ 6.5, while cytochrome $\mathrm{C}$ is most active in a slightly basic environment of $\mathrm{pH} \quad 7.5$. However, both these proteins can function at a neutral pH of 7.0 with little loss in their respective activity. [17] [43] The main issue with $\mathrm{pH}$ is the fact that the ORR found in acidic environments will not proceed at neutral conditions, but instead ORR will use water as its source of hydrogen and produce hydroxyl groups as its final product. This reduces the overall cell potential, as pictured in figure 12, the half cell potential of ORR in acidic conditions is $1.229 \mathrm{~V}$ while under neutral or basic conditions it is 0.401 V. Fortunately, the hydroxyl ions produced at the cathode will readily neutralize the protons

/ hydronium ions produced by PSII and with the use of buffer system the pH of the system should remain neutral. The most pressing issue in the design of such a photoelectrochemical cell is the 


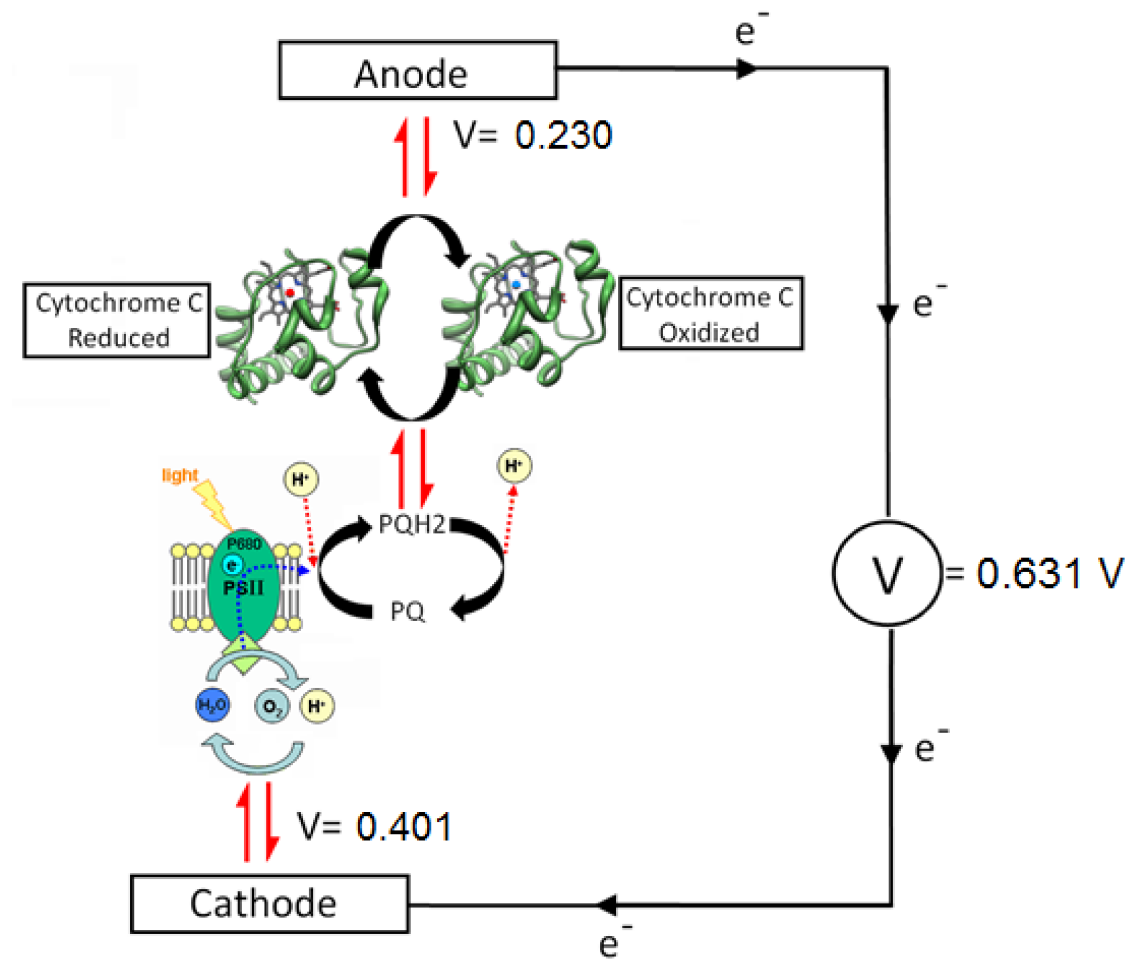

Figure 18: Diagram of a potential photoelectrochemical cell using the REDOX pathway of Photosystem II, plastoquinone and cytochrome C. PSII localized in liposomes along with plastoquinone are illuminated with light allowing oxygen evolution to take place, producing oxygen and protons. The electrons stripped from water are received by plastoquinone, which can then be accepted by cytochrome $\mathrm{C}$ in the solution. The cyotchrome $\mathrm{C}$ can than deliver the electrons to the anode, where they travel through an external circuit and recombine with oxygen and protons to reform water. The cell potential of $0.631 \mathrm{~V}$ is the sum of the anodic and cathodic half cell potentials.

potential for cytochrome $\mathrm{C}$ to accept electrons from the cathode instead of from the lipid bound electron shuttle plastoquinol, forming a short circuit by electrically connecting the cathode and anode through the solution. One way to overcome this is to cover the cathode in a membrane which would separate the cathode from the bulk solution while still allowing oxygen and water to reach the catalyst at the electrodes surface while simultaneously allowing hydroxyl ions to flow away from the cathode and into the bulk solution. Fortunately this problem has already been addressed for cathodes for alkaline and microbial fuel cells which also catalyze ORR at near neutral conditions using what is known as an anion-exchange membrane. [44] In the case of microbial fuel cells these membranes are also designed to reduce biofouling, a phenomena where bio-membranes from cells adhere to the anion exchange membrane and block it from exchanging ions and oxygen with the 
bulk solution. [45]

This type of device has two distinct advantages. First, PSII is housed in lipsomes that emulate the native conditions of a thyalkoid membrane. This should increase the stability and longevity of PSII relative to other photocells that embed their photosynthetic proteins directly to an electrode. Also since PSII is in a lipid bilayer, this can open up the possibility of repairing and replacing proteins that have been damaged by photodecomposition using the biological repair mechanisms found in plant chloroplast. The second advantage is that acceptance of photons is not limited to a two dimensional surface as photovoltiac devices are. The solution that contains the photoelectrochemical cell components can have whatever depth is desired, only being limited by increasingly large surface area requirements for the electrodes. This would potentially allow such a device to absorb virtually all of the photons its is bombarded with, given an approriate depth. 


\section{Extraction Production and Testing of PEC Components}

\subsection{Thylakoid Extraction}

With the exception of cytochrome C, all biologic compounds used in this body of work were extracted from Spinacia oleracea (spinach) purchased fresh from local markets. Spinach leaves are inexpensive, have a high density of chloroplasts in their leaves as noted by their dark green color and are also quite easy to process. Figure 19 shows a simplified workflow for the processing of spinach leaves. Since all of the biologic compounds to be extracted; PSII, plastoquinone and thylakoid lipids, are localized in the thylakoid, the first step is to isolate thylakoids from the leaves.

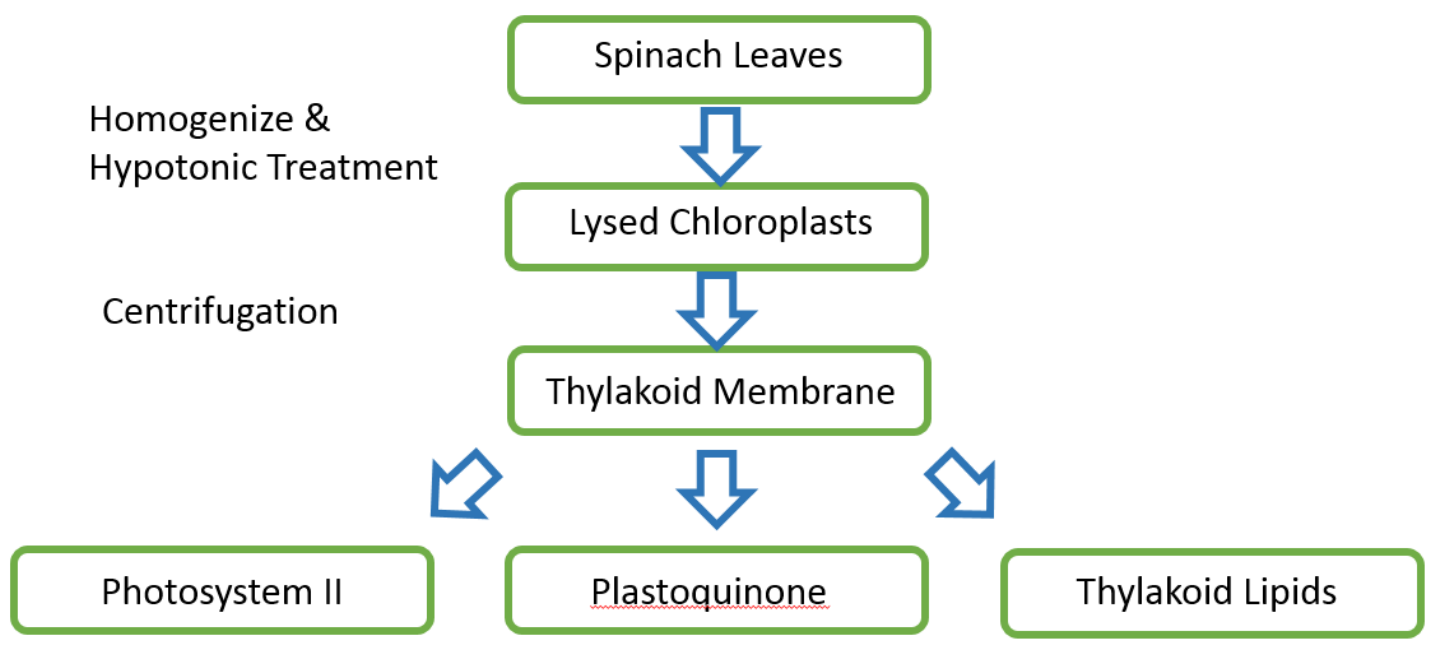

Figure 19: Workflow diagram for processing spinach. Spinach is first homogenized in a blender, filtered and centrifuged at $1000 \mathrm{x} g$ to isolate the chloroplasts. The cloroplasts are solubilized in a hypotonic buffer solution to burst the chloroplasts open. This allows thyalkoid membranes to be isolated using centrifugation at $5000 \mathrm{x}$. Photosystem II, plastoquinone and thyalkoid lipids can then be extracted directly from the spinach thyalkoids.

Based on yield and quantity of buffered solutions required, the most effective procedure for the extraction of thylakoids is as follows. [46] Spinach leaves were depetiolated (stripped of stems), washed in water and weighed. After cutting the leaves in roughly 1-2 $\mathrm{cm}^{2}$ pieces, they were added to a blender along with a buffered solution containing a standard mixture of 50 mM HEPES buffer , $330 \mathrm{mM}$ sucrose, $5 \mathrm{mM} \mathrm{NaCl}$ and $5 \mathrm{mM} \mathrm{MgCl}_{2}$ salts as well as $10 \mathrm{mM}$ ethylenediaminetetraacetic acid (EDTA). HEPES is used as a buffer as it can buffer $\mathrm{pH} 7$ solution while not interfering 
in biochemical reactions. The sucrose is present to maintain an ionic solution strength simular is physiological conditions. $\mathrm{MgCl}_{2}$ is used to maintain protein stability. The $\mathrm{NaCl}$ is present maintain protein solubility. EDTA is present as a chelating agent in order to suppress the activity of metal dependent enzymes, calcium in particular, that can damage proteins. This mixture was then macerated on a high setting using five 1-2 second pulses. The procedure calls for $10 \mathrm{~mL}$ of buffer per one gram of spinach, however using $5 \mathrm{~mL}$ of buffer per gram of spinach yields identical results. The blending breaks the cell walls of the plant cells and releases chloroplasts into the solution. The homogenized spinach leave mixture is then filtered through either miracloth or cheese cloth, using at least two layers of either material, in order to capture any large plant matter that was not fully homogenized during blending. The filtered solution was then centrifuged for five minutes at $1,000 \mathrm{x} g$ in order to separate the chloroplasts from the rest of the solution. Since chloroplasts are relatively large particles on a cellular scale, having a diameter of roughly $5 \mu \mathrm{m}$, when a solution is subject to a low $\mathrm{g}$ spin such as $1,000 \mathrm{x} g$, the chloroplasts will 'pellet out' of the solution and collect as a solid mass at the bottom of a centrifugation tube. The solution in these centrifuge tubes (supernatant) was discarded and the pellet was solubilized by mechanical agitation into the same buffer. Solubilization was done using a glass rod and a complementary glass tube and repeatedly crushing the pellet with the rod in a small amount of buffer solution. The solubilized pellet was than diluted to the same buffer (volume) to spinach (mass) ratio and the centrifugation was then repeated to remove as much cell wall debris and other unwanted cellular artifacts as not to contaminate the pellet. The pellet was then solubilized in a hypotonic buffer solution using the same mechanical agitation, at a volume to spinach mass ratio of $1 \mathrm{~mL}$ buffer per 1 gram of spinach. This hypotonic solution contained only a buffer of $50 \mathrm{mM}$ HEPES, and no other osmolyte such as sucrose, this causes water to flood into the chloroplasts by way of osmosis causing them to rupture and burst. This releases the thylakoids into the solution, thylakoids unlike chloroplasts are not susceptible to hypotonic lysis due to their size and membrane properties. After vortexing the hypotonic solution to ensure even mixing and allowing it to rest for approximately 10 minutes the solution was centrifuged again. However this centrifugation was done at 5,000 $\mathrm{x} g$, in order to pellet out the comparatively smaller thylakoid membranes. As before, the supernatant was discarded and the pellet was solubilized but into a storage buffer. This buffer contains the 20 
mM HEPES, $400 \mathrm{mM}$ sucrose, $15 \mathrm{mM} \mathrm{NaCl}$ and $5 \mathrm{mM} M g C l_{2}$. This solution can be stored for weeks at $-20^{\circ} \mathrm{C}$ or for up to 6 months in a $-80^{\circ} \mathrm{C}$ freezer.

The yield of extracting thylakoid membranes from plant matter is measured in the concentration of chlorophyll. Since chlorophyll is a chromophore and its absorbance spectrum is well known, we can easily assess its concentration spectroscopically. Following the standard procedure from Arnon 1949 [47], a solution containing chlorophyll is diluted in an $80 \%$ solution of acetone and absorbance at 645 and $663 \mathrm{~nm}$ is determined. These wavelengths correspond to chlorophyll b and a respectively, these two analogues these make up the bulk of chlorophyll in plant matter. On top of finding the total amount of chlorophyll in a particular sample, looking at the ratios of chlorophyll a and b can tell us what may be contained in a sample. By using the chlorophyll ratio found in thylakoid membrane as a base line, we can qualitatively assess the extraction of any particular photosystem by looking at how the ratios of chlorophyll a / b change. For example, if one was to try and extract PSII from thylakoid membranes, we would except the final extract to have a higher ratio of chlorophyll a : b than we observed in thylakoid membranes, while a pure extract of PSII containing no PSI or light harvesting complexes would contain almost no chlorophyll b at all.

\subsection{Photosystem II Extraction}

Photosystem II (PSII) can be extracted in a variety of ways, including column chromatography and sucrose density ultra centrifugation. [48] [49] A very simple alternative to these methods to exploit partial solubilization of thylakoid membranes to selectively remove PSI and cytochrome $b_{6} f$ from the thylakoid membrane while leaving PSII. As detailed in a publication by Morrissey et al. 1986 [50], thylakoid membranes are easily solubilized using a non-ionic detergent such as Triton $\mathrm{X}-100$. If a detergent to chlorophyll ratio (mass to mass) is $1: 1$, the thylakoid membrane network will be disrupted, meaning the stacking of thylakoid discs in the form of grana will be destroyed. However this ratio will not solubilize any of the key proteins in the photosynthetic transport chain. As you increase the Triton : chlorophyll ratio however these proteins do begin to be solubilized but not all at once, cytochrome $b_{6} f$ is mostly solubilized $(>90 \%)$ at a ratio of $15: 1$ while PSI 
is mostly solubilized at $20: 1$, however at a ratio of $25: 1$ the majority of PSII ( $>90 \%)$ is still bound to the thylakoid membrane. Therefore if we partially solubilize a thylakoid membrane at a detergent to chlorophyll ratio of $25: 1$, a high g centrifugation will yield a pellet of mostly PSII, while the PSI and cytochrome $b_{6} f$ will be left in the supernatant.

The procedure used here is a modified version of the protocol found in Bricker et. al. 1983. [51] The extraction of thylakoid membranes is the same as done previously, however when extracting PSII it is important to perform all procedures in a dark room at temperature less than $4^{\circ} \mathrm{C}$ and use a green light as illumination. This is to avoid photodecomposition of PSII when majority of its electron acceptor (plastoquinone) is not present and excited electrons produced during hydrolysis have nowhere to flow. After extracting thylakoid membranes and assessing the concentration of chlorophyll, a solution of phenylmethylsulfonyl fluoride (PMSF) was added to the thylakoid solution followed by Triton X-100 at a ratio of $25: 1$ relative to chlorophyll. The PMSF is a protease inhibitor added to avoid damaging the photosynthetic proteins as the thylakoid membranes are broken up. It is important that the concentration of chlorophyll is kept between 1-2 $\mathrm{mg} / \mathrm{mL}$, otherwise the sheer concentration of Triton X-100 required to selectively solubilize the thylakoid membranes will be too great and unexpected results can occur. After mixing in the detergent, the thylakoid solution was lightly agitated using a glass rod for about 30 seconds and then centrifuged at 2,000 $\mathrm{x} g$ for two minutes. This step is to remove any unbroken granum stacks that still persist in the solution as they will pellet out. The supernatant of this initial centrifugation was centrifuged again at $34,000 \times \mathrm{g}$ for ten minutes. The supernatant of this solution, containing the solubilized PSI and cytochrome $b_{6} f$ was discarded and the pellet was broken up in the buffered solution containing no Triton X-100. This step is repeated two more times to remove all the Triton X-100 from the partially solubilized membranes. It is critical that after the addition of Triton X-100 the centrifugation steps are carried out as quickly as safely possible, as prolonged exposure to Triton X-100 will disrupt PSII interactions with its neighboring light harvesting complexes, leading to lower activity. This solution can be stored for short term, about 1 week, in a $-80^{\circ} \mathrm{C}$ freezer or for up to six months if also combined with $30 \%$ glycerol. This PSII containing solution was then analyzed for chlorophyll concentration. Most extractions of thylakoid contained a ratio of chlorophyll a : b of roughly 1.5 , while extractions of PSII contain a ratio of 2.0 or higher. This 
clearly indicates a decrease in the amount of PSI in the extract, while still maintaining a significant amount of the light harvesting complexes that surround PSII. However more detailed analysis is required to confirm this assessment.

\subsubsection{SDS-PAGE Analysis}

Sodium dodecyl sulfate polyacrylamide gel electrophoresis (SDS-PAGE) is a technique commonly used to determine the molecular weight of proteins. Using a cross linked gel of polyacrylamide, a small solution containing a protein(s) of interest is added to the top of a gel. This protein solution is pretreated with sodium dodecyl sulfate (SDS) which both denatures the protein into a single amino acid chain and gives it a uniform charge along the polymer. The charge inferred on the protein by SDS is proportional to the length of the protein and when a mild electrical field is applied across the gel the protein will be forced through the gel matrix. The distance the protein will travel through the gel is directly related to its length, therefore we can separate a mixture of many proteins based on their size and get a picture of what proteins are present. The distance a protein travels in polyacrylamide gel electrophoresis (PAGE) is referred to as its electrophoretic mobility. The gel can be made with varying concentrations of polyacrylamide to cater to what size of protein is analyzed. For example using a high concentration of polyacrylamide such as $20 \%$ we could analyze a mixture of proteins in the 5-30 kDa molecular range, while a concentration of $5 \%$ would be more appropriate for a mixture containing proteins of 50-200 kDa.

The protocol followed in this work is an adaptation of the founding work on SDS-PAGE analysis by Laemmli [52] with modification from Chua et. al. 1980 [53]. In the case of photosynthetic proteins found in thylakoid membranes, the range of molecular weight is roughly $27 \mathrm{kDa}$. In this range it was found that a concentration of $13 \%$ polyacrylamide worked best to resolve the relevant proteins. On top of the usual pretreatment of protein mixtures with SDS, and addition of 6 molar urea was also used as a denaturing agent. The exact mechanism by which urea and SDS combine to denature is not well understood, however it is known to enhance the resolution of low molecular weight proteins at the expense of larger ones (>100 kDa). [54] Instead of the standard coomassie blue stain, a silver stain was used to enhance the visualization of proteins in 


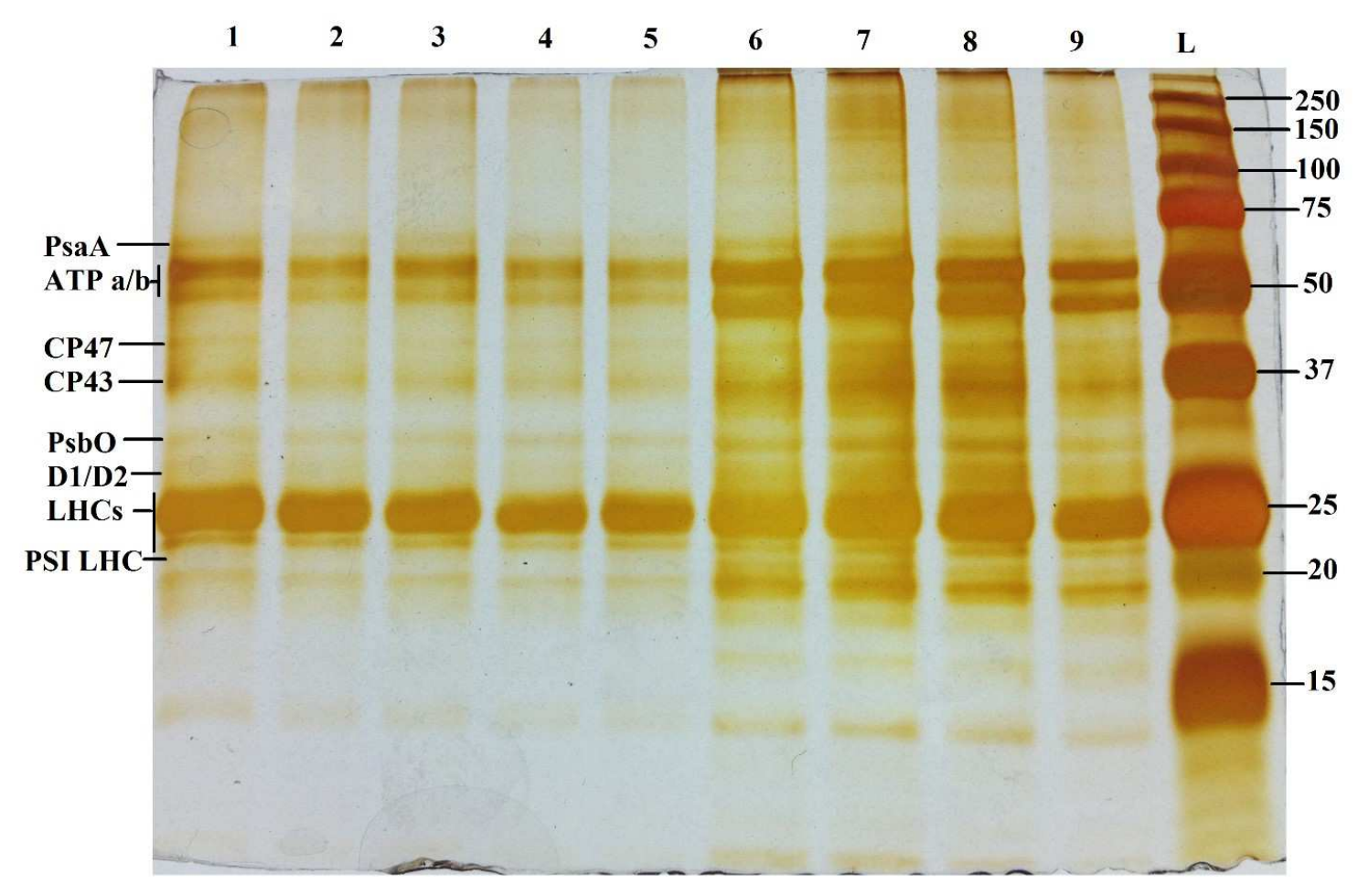

Figure 20: Image of a 13\% SDS-PAGE gel stained with silver. Lanes 1-5 are loaded with Photosystem II extract containing $3.5,2.6,2.6,1.75$, and $1.75 \mu \mathrm{g}$ chlorophyll respectively. Lanes $6-9$ are loaded with thylakoid extract containing 5.3, 3.5, $3.51 .75 \mu \mathrm{g}$ chlorophyll. Lane 'L' contains a standard of ladder proteins. PsaA is part of the Photosystem I protein complex. The proteins CP43/47, PsbO, D1/D2 and LHCs are all part of the PSII protein complex.

the gel. While much more time consuming, a silver ion stain can used to visualize proteins much more precisely as the detection limit of silver is between 0.5-5 ng of protein while coomassie blue requires $>3 \mathrm{ng}$ in order to be effective. [55]

Based on Figure 20, we can clearly see the presence of all the proteins we would expect to find in a PSII extraction. The key indicators of PSII are the CP47, CP43, PsbO, D1/D2 and the LHC proteins. CP47, CP43, D1/D2 are all key proteins making up the core of the PSII complex, PsbO is part of the water oxidizing complex, and the LHCs are the light harvesting complexes surrounding PSII in the thylakoid. However we can also see clear contamination of PsaA in the PSII lanes, meaning some PSI is still present in our extraction. Based on relative intensity of the PsaA bands in the thylakoid lanes and PSII lanes this contamination is not significant. Also supporting this is the lack of PSI light harvesting complexes at $20 \mathrm{kDa}$ in the PSII lanes while the thylakoid membranes show clear banding at $20 \mathrm{kDa}$. Also present are the ATP synthase 
/ subunits in both thylakoid and PSII lanes, which is to be expected in partial solubilization extractions such as this. The ATP synthase subunits pose no complications in terms of activity for either photosystem.

\subsubsection{Photosystem II Activity and Oxygen Evolution}

Since the main function of PSII is to perform hydrolysis, the standard method for finding the activity of PSII is to measure the rate of $O_{2}$ production when exposed to light. This is done using a specialized oxygen electrode known as a Clark type oxygen electrode, as seen in Figure 21. The basic principle is an electrode is placed into a solution with the working electrode separated from the solution by a thin layer of Teflon, permeable only to oxygen. An oxygen reduction reaction occurs at the working electrode and the amount of current consumed by the electrode is used to determine the concentration of oxygen in the solution.

The following is a standard protocol used for investigating oxygen evolution in solutions containing active PSII. [48] A YSI 530 Biological Oxygen Monitor was used to determine oxygen concentration, along with a $60 \mathrm{~W}$ halogen light bulb placed no more than $15 \mathrm{~cm}$ away from the reaction vessel to provide illumination. The reaction solution used two electron acceptors for PSII, $50 \mu \mathrm{M}$ 2,6-Dichloro-1,4-benzoquinone (DCBQ) and $1 \mathrm{mM} K F e(C N)_{6}$. The DCBQ acts as a plastoquinone analogue while the $K F e(C N)_{6}$ keeps the DCBQ oxidized. For comparison both thylakoid membrane and PSII extracts were tested. Photosynthetic oxygen evolution is measured in terms of moles of oxygen produced per unit time and mass of chlorophyll, the standard unit is $\mu$ Mole $\mathrm{O}_{2} / \mathrm{mg}$ Chlorophyll $\cdot$ hour. The thylakoid membranes showed an oxygen activity of $70 \mu$ Mole $O_{2} / \mathrm{mg}$ Chl.hr while the PSII extraction showed an activity of $175 \mu$ Mole $O_{2} / \mathrm{mg}$ Chl.hr. While this activity was modest compared to PSII extracted using sucrose density ultra centrifugation, which can reach over $1,000 \mu$ Mole $O_{2} / \mathrm{mg}$ Chl-hr. However the results were on par with the activity found in publications using the partial thylakoid solubilization in order to isolate PSII. [48] [51] 


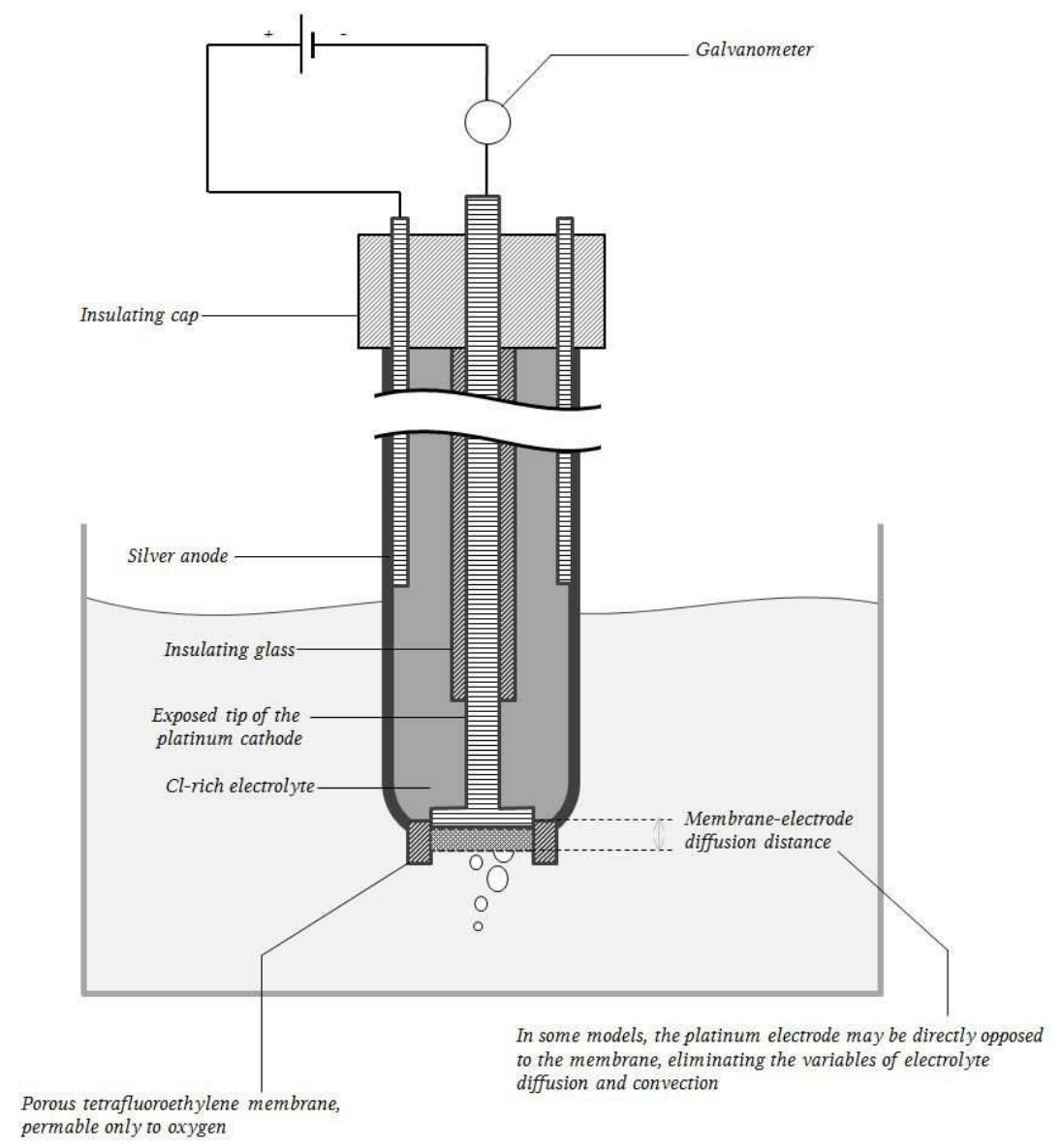

Figure 21: Simplfied diagram of a Clark type $O_{2}$ electrode.[56] The membrane at the tip of the probe selectively allows oxygen through. A platinum cathode reduces the oxygen and based on the current consumed by this reaction, the concentration of oxygen in the solution can be determined.

\subsubsection{Conclusion}

The oxygen evolution of the PSII solution was well over double that of the thylakoid solution. Since the activity was measured in terms of the amount of chlorophyll present and that PSI will not contribute at all to $\mathrm{O}_{2}$ evolution, the PSII extracts increased oxygen evolution clearly indicated a higher proportion of PSII per unit chlorophyll than the thylakoid extract. Combined with the change chlorophyll a / b ratios and the SDS-PAGE analysis, it is evident that this solution did in fact contain an enriched PSII mixture. 


\subsection{Plastoquinone Extraction}

Plastoquinone pictured in Figure 22, was extracted using a modified protocol from Barr et. al. 1967. [57] Starting with freshly extract thylakoid membranes from spinach leaves, the first step in plastoquinone extraction was to separate the organic or non-polar molecules from the proteins and other water soluble compounds using liquid-liquid extraction. A thylakoid membrane solution, diluted to $1 \mathrm{mg}$ chlorophyll / $\mathrm{mL}$ was added to a solution of hexane and iso-propanol at a volume ratio of $4: 5: 5$, thylakoid : hexane : iso-propanol. This solution was shaken for about an hour in order to fully solubilize all of the thylakoid membranes. Using a separatory funnel, the dark green epiphase (low density fraction) containing the majority of the organic soluble compounds was separated from the hypophase (high density fraction) which contained the water soluble compounds. The remaining organic soluble compounds in the aqueous hypophase were removed by repeatedly washing the aqueous phase against a solution of $1: 2: 1$, hexane : acetone : toluene, until all of the green color of the hypophase was gone. All of the organic epiphase fractions were combined together and filtered through a packed column of celite in order to remove any particulates, such as aggregated proteins, left over from the liquid-liquid extraction. After filtration the organic epiphases were extracted against an equal volume $50 \%$ ethanol in water and shaken for at least ten minutes in order to separate the glycolipids from the chlorophyll and plastoquinone. Without this step plastoquinone would exhibit irregular and unpredictable Rfs on silica matrices, making separation by column chromatography extremely difficult. After separating the organic epiphase from the ethanol solution, the epiphase was treated with sodium sulfate in order to remove any residual water. Finally solvent of this solution was removed using a rotary evaporator, and its dry mass was determined.

Column chromatography was used to isolated plastoquinone from the myriad of other organic soluble molecules present in the liquid-liquid extract. Based on the guidelines set out by Still et. al. 1978, [30] an appropriately sized flash column was packed with 40 - 60 uM SiliaFlash silica. Based on the procedure for extracting synthetized plastoquinone from Boers et. al. [58], a solvent system of diethylether in hexane was chosen, and the column was preconditioned using a $1 \%$ solution of ether in hexane. Using a minimal amount of $1 \%$ ether in hexane, the organic 
<smiles>CCCCC1=CC(=O)C(C)=C(C)C1=O</smiles><smiles>[R]C1=CC(=O)C(C)=C(C)C1=O</smiles><smiles>[R]c1cc(O)c(C)c(C)c1O</smiles>

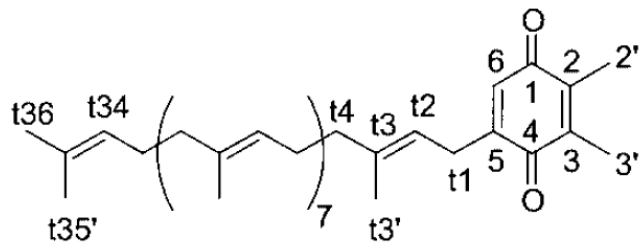

$\mathrm{t} 35^{\prime}$

Figure 22: Diagram showing the molecular structure of plastoquinone (left), REDOX activity of PQ showing plastoquinol in equilibrium with plastoquinone (center), and an annotated version of plastoquinones structure (right).[58]

extract from the liquid-liquid extraction was loaded onto the flash column. One full column volume of $1 \%$ ether in hexane was run through the column, removing the low polarity compounds such as $\beta$-carotene and vitamin $K_{1}$. The solvent was then switched to $2 \%$ ether in hexane, which quickly eluted out plastoquinone. The eluate composition was tracked using small $5 \mathrm{~cm}$ TLC plates running $2 \%$ ether in hexane. Plastoquinone was found to have an Rf of 0.3 in such a solvent system and could be easily visualized using a UV lamp due to its conjugated ring structure. Fractions that contained plastoquinone were pooled together and concentrated using rotary evaporation.

\subsubsection{Plastoquinone NMR Analysis}

Nuclear magnetic resonance (NMR) was used to confirm the structure of our perspective plastoquinone extractions. Plastoquinone was dissolved in a solution deuterated chloroform containing a trace amount of tetramethylsilane (TMS) as an internal standard with which to calibrate the chemical shifts of the analyte. The scans were performed on a 'Bruker' $300 \mathrm{MHz} \mathrm{NMR}$ set for 1D proton analysis. Figures 23 and 24 show the NMR spectrum of a sample of PQ as well as the relevant peaks and integration.

The NMR spectrum obtained was evaluated relative to results from synthesized plastoquinone in Boers et. al. [58] The signature peak of plastoquinone is found at $6.465 \mathrm{ppm}$, a triplet 


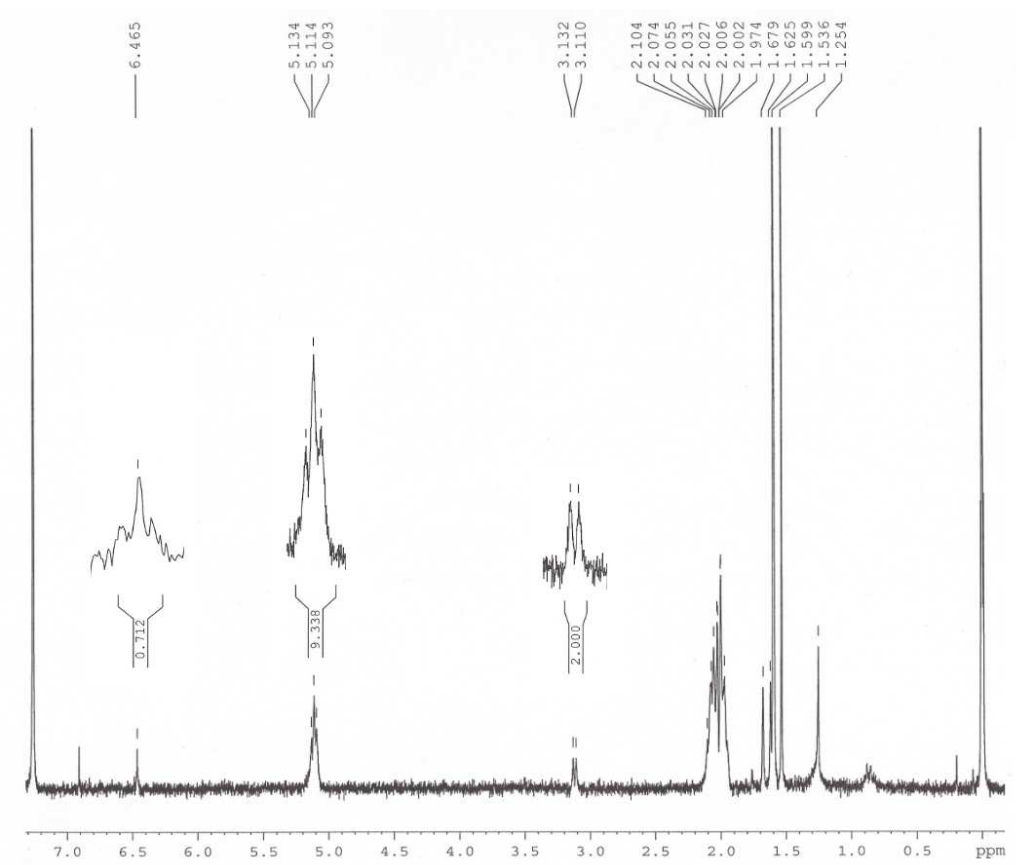

Figure 23: Total NMR Spectrum of Plastoquinone column extract. Peaks at 6.47, 5.10, and 3.12 ppm are expanded on the spectra to highlight their spin states.

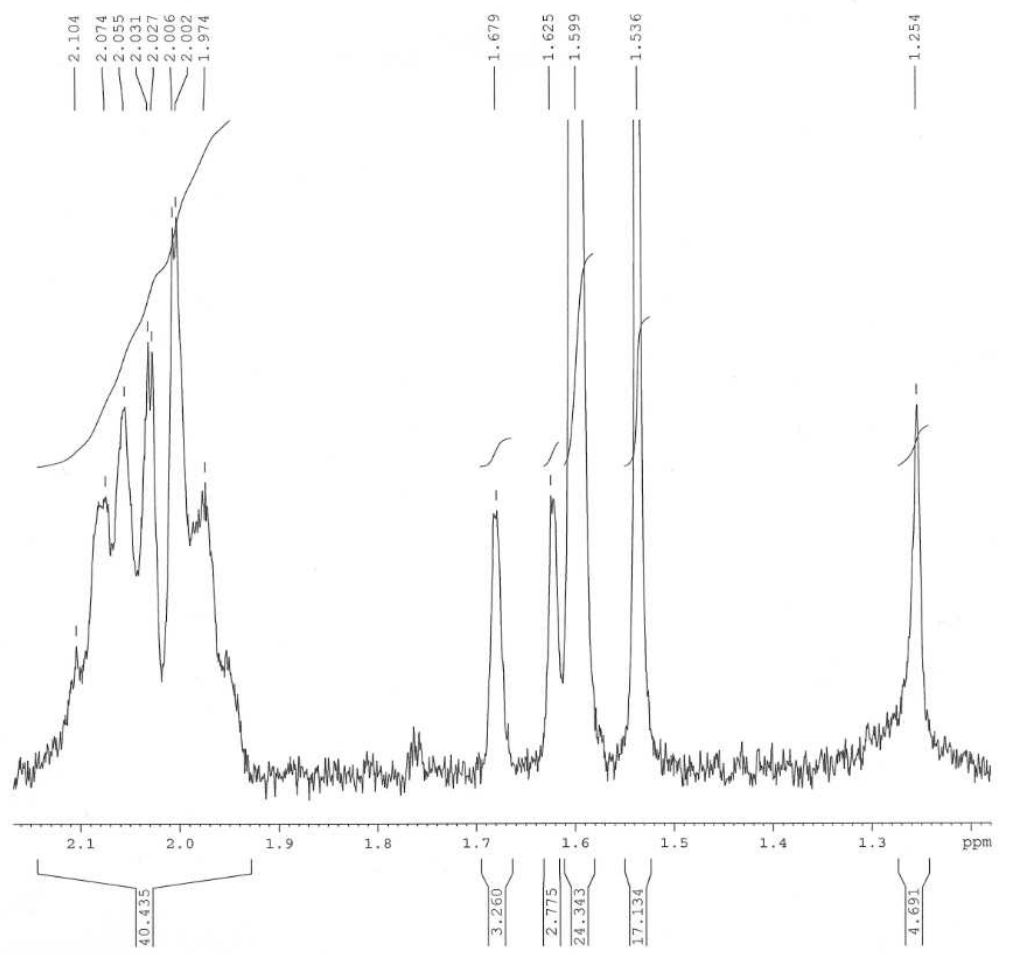

Figure 24: NMR spectrum of Plastoquinone showing 1-2 ppm. This region makes up the alkane region for NMR analysis. 
formed by the 6th carbon of the quinone ring. It is considered a signature peak as its location is in an area where chemical shifts are relatively rare, being in the upper range of where vinyl protons appear. Its integration was under 1.0, meaning the signal was weak, possibly due to the fact that its coupling is long range, with the two protons on the t1 carbon. The peaks at $5.13-5.09$ are actually three separate triplets of the vinyl protons along the isoprene tail of PQ. The vinyl protons of the first and last isoprene units was overwhelmed by the signal of the 7 vinyl protons along the center of the isoprene chain, meaning that distinguishing between protons on $\mathrm{t} 2$, $\mathrm{t} 34$ and central protons was very difficult. However the integration read as 9.3 protons, which is quite close to the excepted value of 9 . The next peak of interest was the doublet found at 3.12, existing at the edge of the range where alkane protons appear. The 3.12 peak is produced by the two protons on the t1 carbon interacting with the single vinyl proton on t2. The reason its ppm is so high is due to upfield shifting caused by its proximity to the quinone ring. This peak was used as the standard for integration, hence its value is 2.000 . The multiplet of peaks at $1.98-2.06$ comprise the slightly upshifted alkane protons, this region is dominate by the $\mathrm{C}-\mathrm{H} 2$ alkane protons of the isoprene chain, comprising 32 protons starting from carbon $t 4$ to t33. Also present are the protons of the methyl groups of the quinone ring, denoted as carbon 2' and 3'. All together this would make 38 protons, the integration reads as 40.3 which is not far off considering the scale of this signal. Finally we have the peaks at $1.5-1.7$, sitting in the center of the alkane proton region. The peak at 1.68 with an integration of 3.26 denotes the protons on the trans t36 carbon, this integration is quite close to the excepted value of 3 . We would expect to see a signal at 1.58 for the cis t35' carbon however this peak seems to have been overwhelmed by the 1.60 peak from the seven methyl group protons along the isoprene chain. This massive peak at 1.60 has an integration of 24.3 , counting the 21 protons from the seven central isoprene units along the chain, and adding the 3 more for the protons on the cis t35' carbon, this value is in line with expected results. The results of NMR analysis on the PQ extracts shows high fidelity with synthetically produced $\mathrm{PQ}$, showing the $\mathrm{PQ}$ extract to be high in purity and without any significant difference in molecular structure. 


\subsubsection{Plastoquinone Quantitative Analysis}

Precise concentration of plastoquinone can be easily determined using spectroscopic analysis. When dissolved in anhydrous ethanol plastoquinone and its reduced analogue plastoquinol, exhibit a marked and predictable difference in their absorbance spectra. [57] Plastoquinone is found to have a local peak of absorbance in ethanol at $255 \mathrm{~nm}$, however when such a solution is reduced to form plastoquinol, this absorbance drops. The difference in plastoquinone and plastoquinol absorbance at $255 \mathrm{~nm}$ correlates to the concentration of total PQ by a molar extinction coefficient of $15 \mu \mathrm{M} / \mathrm{cm}$.

Starting with solid sample of plastoquinone, a known mass of PQ was dissolved in ethanol and analyzed using a Varian Cary 3 UV-Vis Spectrophotometer. The solution was diluted until the absorbance at $255 \mathrm{~nm}$ was between 0.5 - 1. Next a small amount of sodium borohydride was added to the PQ-ethanol solution, mixed for roughly ten seconds and then filtered through a pipette stuffed with cotton to remove any residual $\mathrm{NaBH}_{4}$. This step needs to be carried out quickly in order to avoid oxidation from exposure to oxygen in the air. The absorbance at $255 \mathrm{~nm}$ was then determined. Using the difference in absorbance at $255 \mathrm{~nm}$ between plastoquinone and plastoquinol and applying the known molar extinction coefficient for this process, the purity of PQ samples was determined to $60-70 \%$ in most samples. However after extended $(>1 \mathrm{hr})$ exposure to high vacuums this purity was increased to $90 \%$ or greater.

\subsection{Thylakoid Lipid Extraction}

Thylakoid lipids were isolated using preparatory thin layer chromatography pTLC, using modified silica plates.[59] Preparative TLC plates with $1000 \mu \mathrm{M}$ thickness with dimensions of $20 \mathrm{x} 20 \mathrm{~cm}$ (Silicycle, SiliaPlate) were impregnated with ammonium sulfate by carefully submerging the entire plate into $20 \%$ solution of ammonium sulfate in water for approximately 30 seconds. The TLC plate was then allowed to rest in an enclosed container for at least 48 hours, after which the plates were heated at $120^{\circ} \mathrm{C}$ for two hours. By applying heat the ammonium sublimates off of the plate leaving behind bisulfate which is now embedded into the silica matrix. The conjugate base of sulfuric acid modifies the $\mathrm{Rf}$ of the lipids by interacting with the lipid headgroups, allowing for 
total separation of lipids comprising a thylakoid membrane.

Thylakoid extract was prepared for loading onto pTLC plates

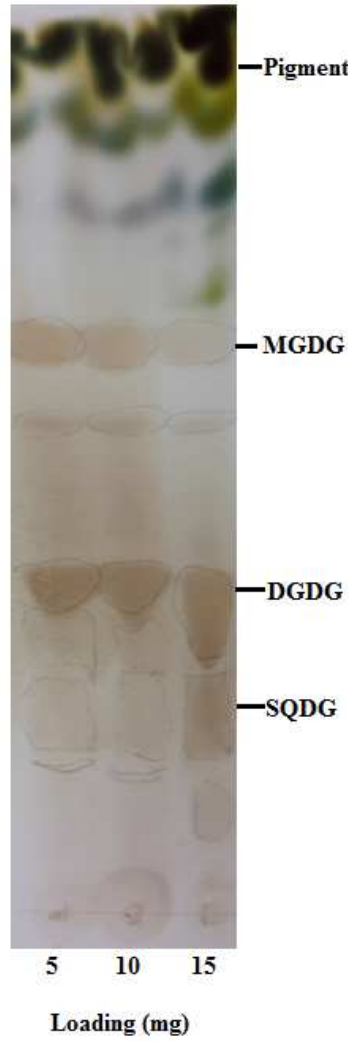

Figure 25: Image showing a TLC of thylakoid lipids on a $250 \mu \mathrm{m}$ plate embedded with bisulfate. MGDG, DGDG, and SQDG are the key lipids contained in thyalkoid membranes of spinach chloroplasts. using the following method. [59] For every milligram of chlorophyll in a thylakoid extract solution, $20 \mathrm{~mL}$ of $20: 10: 1$ methanol, chloroform and formic acid was added and the solution was shaken vigorously. This simultaneously breaks up the thylakoid membranes and denatures any enzymes capable of acting upon the lipids. Next a half a volume of $0.2 \mathrm{M}$ $\mathrm{H}_{3} \mathrm{PO}_{4}$ and $1 \mathrm{M} \mathrm{NaCl}$ was added to the solution and the chloroform hypophase was removed using a separatory funnel. The chloroform phase was evaporated to dryness using a rotary evaporator and the mass of organic extract was weighed. A small volume of chloroform was added to solubilize the extract for storage or pTLC loading.

The maximum loading for a silica TLC with a thickness of 250 $\mu \mathrm{M}$ was found to be approximately $10 \mathrm{mg}$ of thylakoid organic extract per $1 \mathrm{~cm}^{2}$. At loadings of greater than $10 \mathrm{mg}$ thylakoid extract per 1 $\mathrm{cm}^{2}$ lipid bands begin to streak and overlap with one another, as seen in Figure 25 where the DGDG band is starting to overlap with the SQDG. Based on the general rule for pTLC stating that the loading capacity of TLC increases proportionally to square root of the increase in thickness, a $1000 \mu \mathrm{M}$ thickness pTLC plate would have a maximum loading of roughly $20 \mathrm{mg}$ of thylakoid organic extract per $1 \mathrm{~cm}^{2}$. The solvent system used to run the pTLC was a solution of $91 \mathrm{~mL}$ acetone, $30 \mathrm{~mL}$ toluene and $7.0 \mathrm{~mL}$ of $d \mathrm{H}_{2} \mathrm{O}$. The spots were visualized using iodine crystals, Rf values for MGDG, DGDG and SQDG matched expected results well. Perspective lipids were scrapped off of the pTLC plate and a solution of methanol was used to remove the lipids off of the silica gel via filtration. The samples were then evaporated to dryness using a rotary evaporator, followed by at least one hour on in a high vacuum system to remove any residual solvents left over. 


\subsubsection{Thylakoid Lipid NMR Analysis}

NMR analysis was performed on the thylakoid lipid extracts in order to confirm the molecular makeup of each perspective lipid. However unlike with plastoquinone, each extracted lipids contain a mixture of different fatty acid chains, meaning assignment of peaks and integration is not practical or useful. NMR in this case was used simply to confirm the identity of each lipid based on its headgroup and signature NMR peak. For comparison, NMR analysis of thylakoid lipids extracted from microalgae [60] were used to confirm the identity of the extract lipids. Lipid samples used for NMR analysis were dissolved in a $1: 1$ solution of deuterated chloroform and methanol with TMS as an internal calibration standard.

Table 1 shows the excepted values for signature peaks in each lipid, as well as the values found for our extracted lipids. The data on signature peaks clearly showed that the pTLC extracted lipids were in fact present. Also the fidelity between the excepted and obtained NMR spectra, as in Figures 26, 27, and 28 was very high especially in the alkane and alkene chemical shift regions. This indicates that the fatty acid composition of these lipids is very similar. Based on the pTLC banding patterns and the NMR data presented here, it can be stated with some confidence that the thylakoid lipid extraction did in fact produce MGDG, DGDG and SQDG.

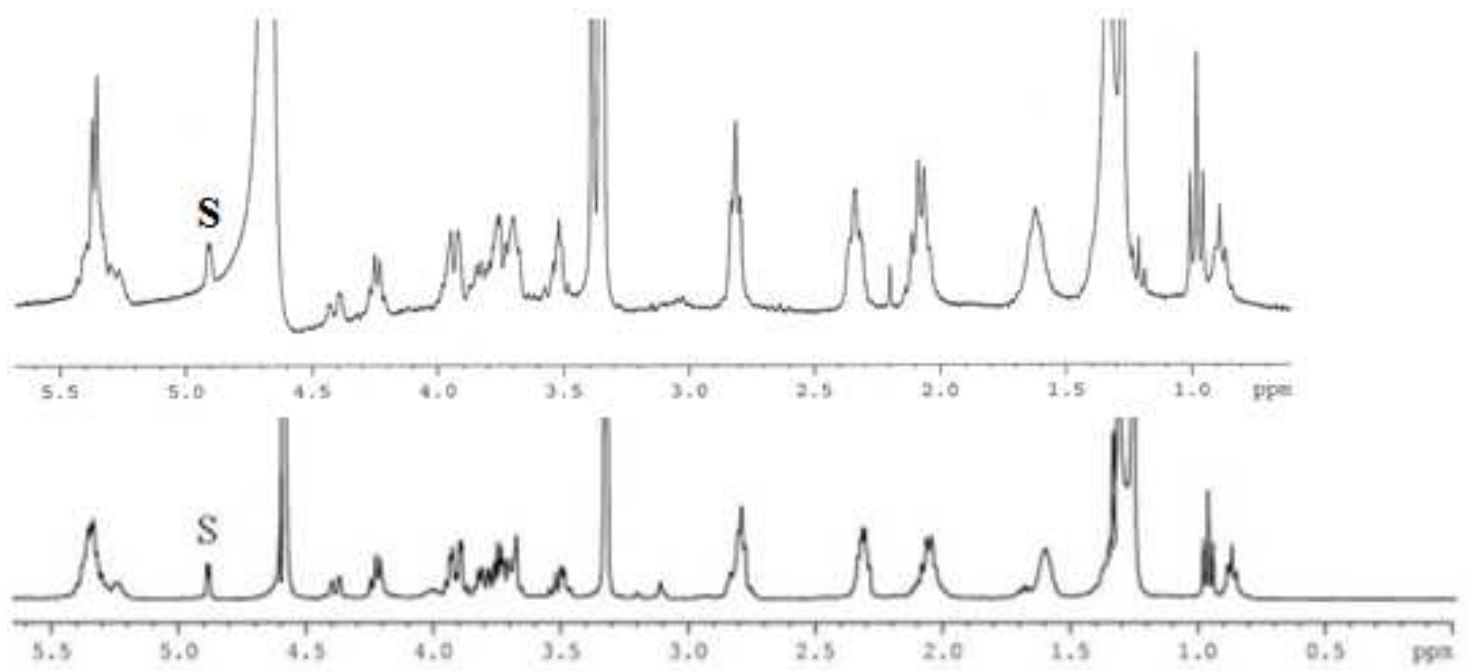

Figure 26: Images of extracted DGDG lipids NMR spectrum (top) and comparison spectrum of the same lipid from microalgae (bottom) [60]. 


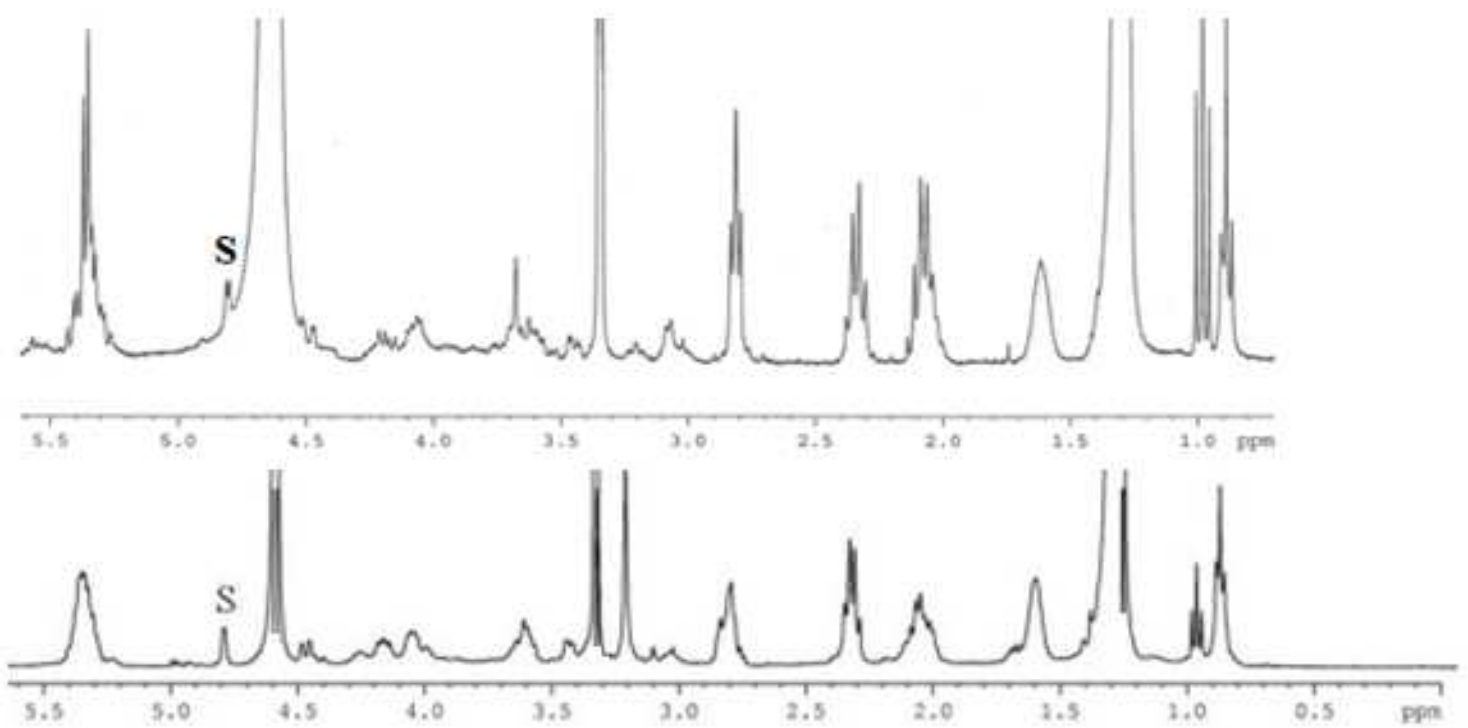

Figure 27: Images of extracted SQDG lipids NMR spectrum (top) and comparison spectrum of the same lipid from microalgae (bottom) [60].
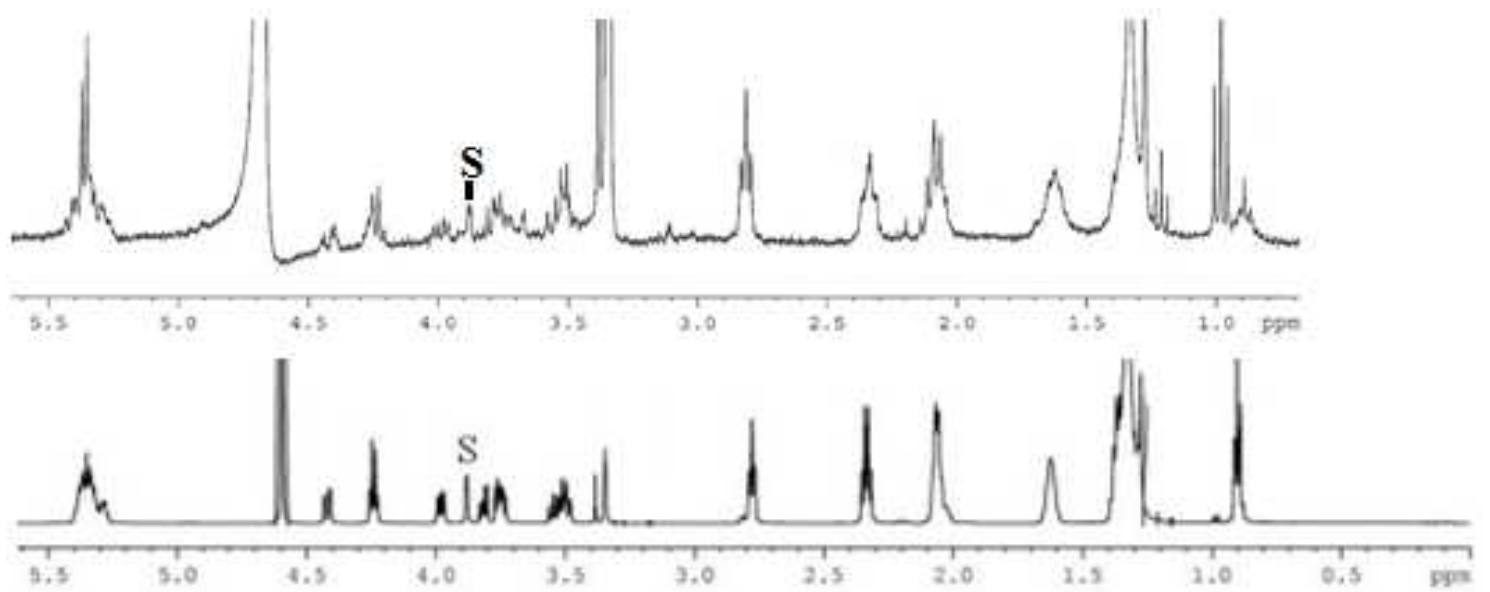

Figure 28: Images of extracted MGDG lipids NMR spectrum (top) and comparison spectrum of the same lipid from microalgae (bottom) [60]. 


\begin{tabular}{|c|c|c|c|c|c|}
\hline Lipid Class & $\begin{array}{c}\text { Signature Peak } \\
\text { Chemical Assignment }\end{array}$ & $\begin{array}{c}\text { Excepted } \\
\text { Chemical Shift } \\
\text { (ppm) }\end{array}$ & $\begin{array}{l}\text { Extracted Lipid } \\
\text { Chemical Shift } \\
\text { (ppm) }\end{array}$ & $\begin{array}{c}\text { Excepted } \\
\text { Coupling } \\
\text { Constant } \\
(\mathrm{Hz})\end{array}$ & $\begin{array}{c}\text { Extracted } \\
\text { Lipid } \\
\text { Coupling } \\
\text { Constant } \\
(\mathrm{Hz})\end{array}$ \\
\hline SQDG & $\begin{array}{c}\text { Anomeric proton of } \\
\text { sulfoquinovoside headgroup }\end{array}$ & $4.80(\mathrm{~d})$ & $4.81(\mathrm{~d})$ & 3.7 & 3.3 \\
\hline DGDG & $\begin{array}{l}\text { Anomeric proton of } \\
\text { galactose headgroup }\end{array}$ & $4.90(\mathrm{~d})$ & $4.91(\mathrm{~d})$ & 3.5 & 3.0 \\
\hline MGDG & $\begin{array}{c}\text { Carbon } \# 4 \text { of galactose } \\
\text { headgroup }\end{array}$ & 3.88 (d) & 3.88 (d) & 3.0 & 2.8 \\
\hline
\end{tabular}

Table 1: Comparing excepted chemical shifts and coupling constants for 'signature' peaks on thylakoid lipids from microalgae against thyalkoid lipids extracted from spinach.[60]

\section{$2.5 \omega$-Hydroxythiol SAM Anode Production}

Gold electrodes consisting of a glass backing with $10 \mu \mathrm{m}$ of chromium and $100 \mu \mathrm{m}$ of gold were used as a substrate. The gold surface was functionalized with a $\omega$-Hydroxythiol self-assembled monolayer (SAM) using the procedure found in Becka, Miller 1992. [61] The first step was to clean the Au surface of the electrode, using an RCA cleaning protocol to remove any organic residue. A solution of 5 parts DI water, 1 part ammonium hydroxide ( $30 \%$ ) and 1 part hydrogen peroxide $(35 \%)$ was prepared. Using a hotplate this solution was heated to $70-80{ }^{\circ} \mathrm{C}$, where it began to bubble vigorously. The Au electrode was placed in the solution for 10 minutes followed by repeated washing using DI water. Next the electrode was placed into a solution of $10 \mathrm{mM}$ 2-Mercaptoethanol ( $\beta$-Met) for at least 24 hours. The $\beta$-Met solution was deaerated using either $N_{2}$ or $A r$ gas prior to submerging the cleaned $\mathrm{Au}$ electrode. The solution was kept out of direct light during the 24 hour incubation with the electrode and light magnetic stirring was applied to keep the solution homogenous. The thiol groups of the $\beta$-Met will spontaneously bond to the cleaned Au surface, forming the desired SAM with a hydroxyl surface. [22] [24] 


\subsection{Anion Exchange Membrane Production}

An anion exchange membrane for separation of the cathode surface from the bulk solution was made using crosslinked polyvinyl alcohol (PVA) and polydiallyldimethyl ammonium chloride (PDDA). PVA crosslinked with glutaraldehyde (GA) using catalytic amounts of acid acts as a mesh which holds PDDA in place, as seen in Figure 29. [45] The quaternary ammonium cation of PDDA allows hydroxyl anions to flow in and out of the membrane. This system provides a very simple and cost effective method of producing anion exchange membranes for alkaline and microbial fuel cells.<smiles>CC(C)(C)CC(O)C(C)(C)C</smiles>

(PVA)

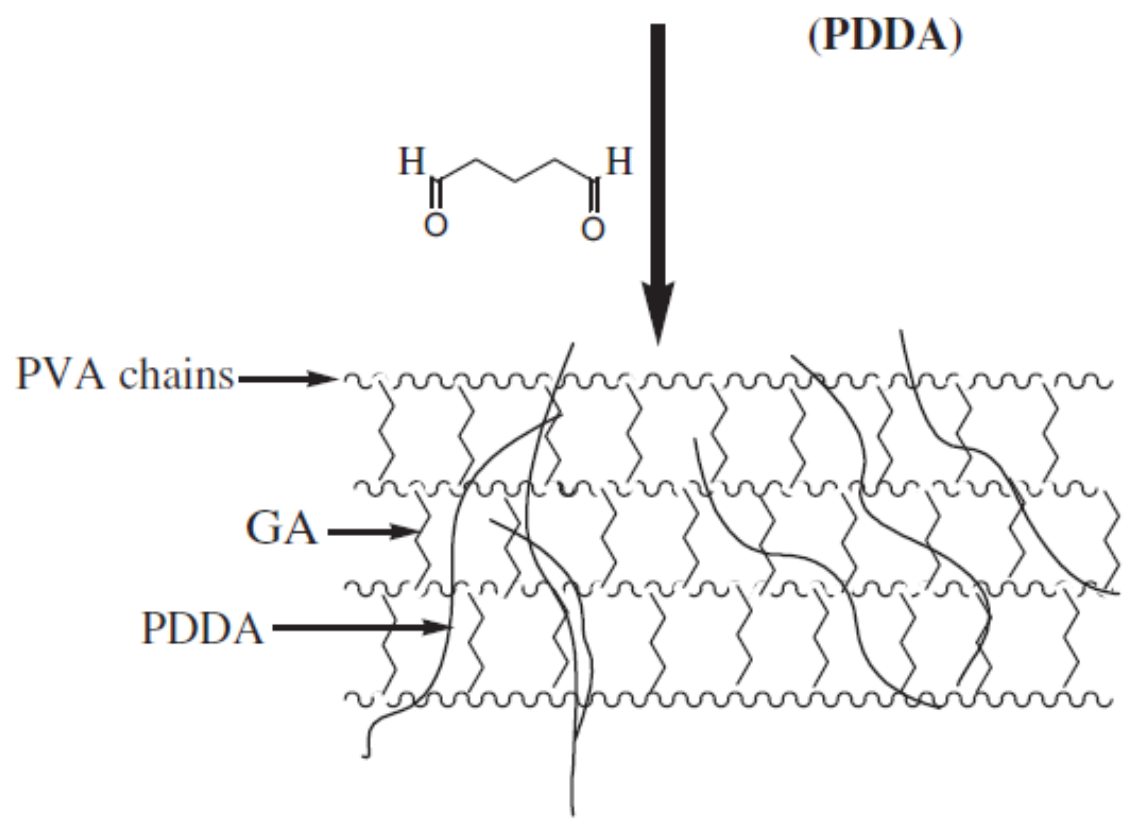

Figure 29: Diagram of PVA-PDDA membrane production, showing PVA (polyvinyl alcohol), PDDA (polydiallyldimethytl ammonium chloride) and GA (glutaraldehyde) before and after crosslinking treatment.[45]

The first step in the protocol was to produce an aqueous solution of PVA. 78,000 MW 98\% hydrolyzed PVA was dissolved in water first mixing $125 \mathrm{mg}$ in $5 \mathrm{~mL}$ of ice bath cooled distilled water and mixed for 10 mins using light magnetic stirring. The solution was then heated to $90^{\circ} \mathrm{C}$ using an oil bath with continuous mixing until the solution was completely transparent. 
This solution was allowed to cool to room temperature before $1.25 \mathrm{~mL} 20 \%$ solution of medium molecular weight ( $200-300 \mathrm{kDa}$ ) PDDA was added, making a 1: 1 ratio of PVA : PDDA by mass. This solution was mixed for at least 3 hours to ensure a homogenous mixture of PVA-PDDA prior to crosslinking. An aqueous solution of $50 \%$ GA was added to produce a final concentration of $10 \%$ mass / volume, this solution was mixed for 30 minutes. The crosslinking was catalyzed using a small addition of diluted $\mathrm{HCl}$. Using $2 \mathrm{~mL}$ fractions of the PVA, PDDA, GA solution, $27 \mu \mathrm{L}$ of $3.7 \% \mathrm{HCl}$ was added and the solution was mixed for 2 minutes prior to casting into a petri dishes. The membranes quickly hardened on the petri dishes and within 10 minutes became extremely viscous. The cast membrane solutions were left for at least 12 hours at room temperature to dry. The hardened membranes were removed from the petri dishes by adding $d \mathrm{H}_{2} \mathrm{O}$ which hydrated the membranes and allowed them to be removed from the pyrex surface of the dish. Using electronic calipers the dry thickness of the films was found to be between 50-100 $\mu \mathrm{m}$, depending on how they

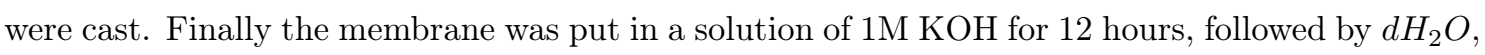
to exchange the chloride ions on the quaternary amine with hydroxyl groups, to prime it for use as an anion exchange membrane. The final results is show in Figure 30.

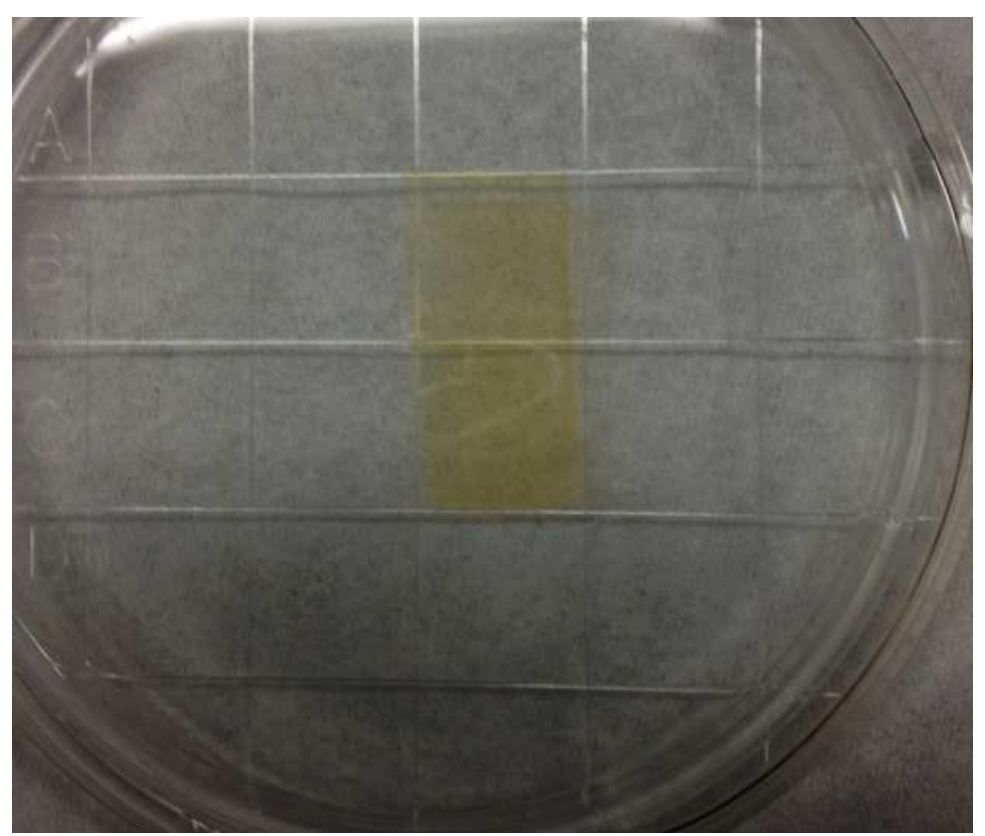

Figure 30: An image of PVA-PDDA anion exchange membrane after crosslinking and KOH treatment, resting in $\mathrm{dH}_{2} \mathrm{O}$. 


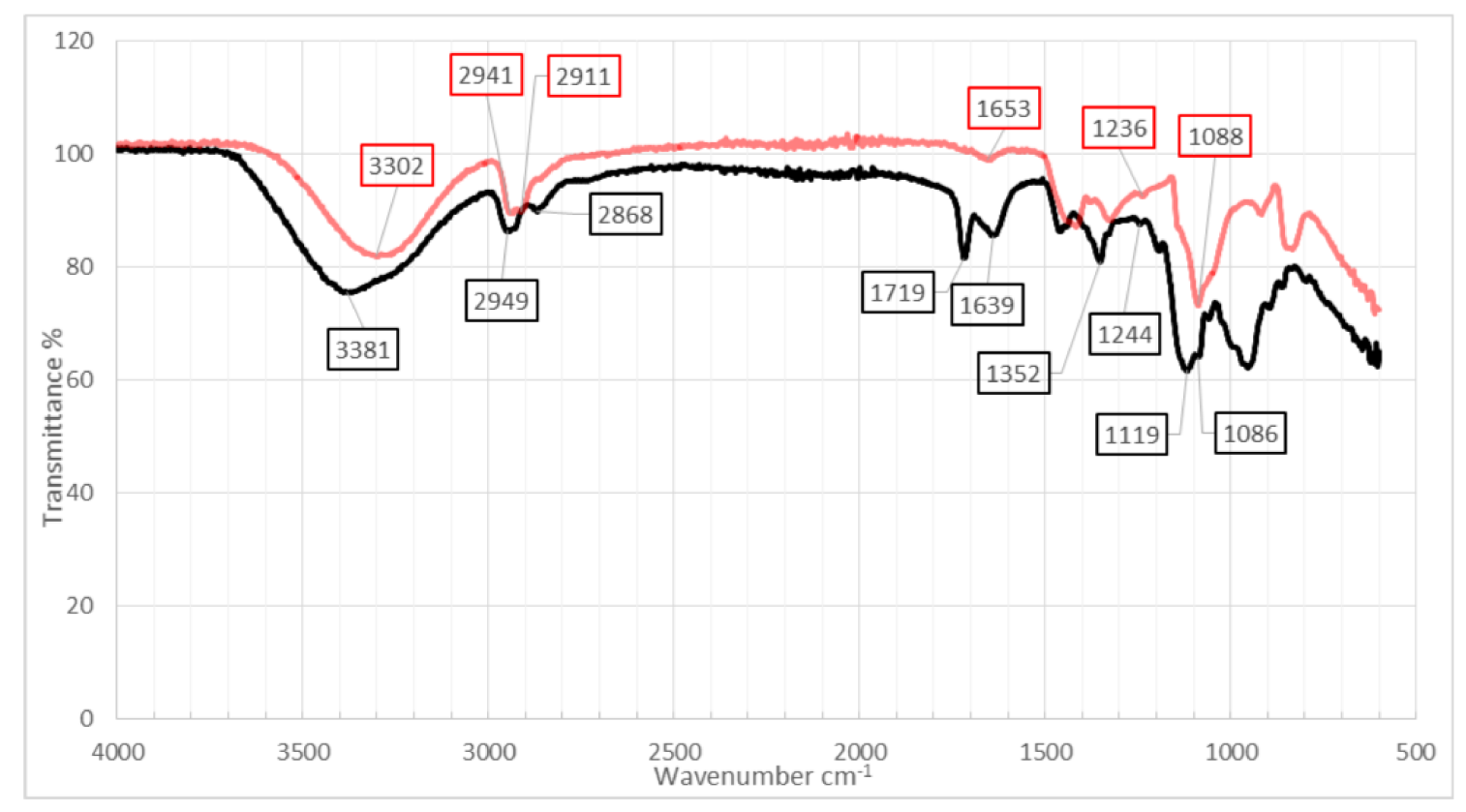

Figure 31: The red (upper) line shows ATR-IR Spectrum of pretreatment PVA-PDDA without GA and the black (lower) line shows ATR-IR Spectrum post crosslinking treatment PVA-PDDA (black line). The key peaks on the post treatment spectrum are 2868 and 1119 , showing $C-H_{2}$ and $C-O$ stretching respectively. These peaks are specific to GA crosslinked to PVA.

\subsubsection{Anion Exchange Membrane ATR-IR Analysis}

Attenuated total reflection infrared spectroscopy was performed on the anion exchange membrane in order to confirm PVA crosslinking and the inclusion of PDDA in the membrane. IR spectroscopy was performed using a PIKE Technologies MIRacle Single Reflection ATR setup on a Varian Scimitar 1000 FT-IR spectrometer. Analysis was done on GA treated and crosslinked membranes, as well as PVA-PDDA only membranes to see the effects of crosslinking treatment. Figure 31 shows the transmittance of both membranes. The peaks at 3381 and 3302 on treated and non-treated membranes are from hydroxyl group stretches on the PVA, and potentially from any water trapped in the crosslinked membrane in the case of treated membranes. The peaks at 2800-2900 are C$\mathrm{H}$ bond stretching, with the peak at 2868 on the treated membrane being a product of $\mathrm{C}-\mathrm{H}_{2}$ groups on crosslinked GA. The 1719 peak of the treated membrane is from $C=O$ stretching on unreacted aldehydes of GA. There is some debate in the literature about the peaks at 1653 and 1639 for untreated and treated PVA-PDDA membranes. They may be unreacted carbonyl groups left over from PVA production or possibly $C-O$ stretches in crosslinked GA in the case of treated 
membranes. The peaks at 1300-1400 are from $C-H$ and $O-H$ bending in both PVA and PDDA. The peaks at 1236 and 1244 for untreated and treated membranes are likely $C-N$ stretches of the quaternary amine in PDDA, this peak is generally weak in tertiary and quaternary amines. The peaks at 1100 are from $C-O$ stretches, with the peak at 1119 , only appearing in treated membranes, being characteristic of PVA crosslinked with GA.

\subsection{Platinum on Carbon Cathode Production}

The cathodes used in this body of work use an Au electrode with platinum on graphitized carbon $(\mathrm{Pt} / \mathrm{C})$ as a catalyst, with polyvinyl alcohol (PVA) as a binder, combined with a PVA / PDDA anion exchange membrane hot pressed to the electrode using a graphite epoxy. [45] [62] The first step in cathode production was to produce a $\mathrm{Pt} / \mathrm{C}$ ink that could be sprayed onto an electrode or anion exchange membrane. $800 \mu \mathrm{L}$ of a 1\% PVA solution was diluted with $10 \mathrm{~mL}$ of $1: 1$ acetone isopropanol. Then $8 \mathrm{mg}$ of $20 \% \mathrm{Pt}$ on Vulcan XC72 graphite was added to the solution followed by sonication for at least one hour. This ink solution had a PVA : Pt/C ratio of $1: 1$ by mass. Using a gravity fed spray gun, operating at roughly $20 \mathrm{kPa}$ of $N_{2}$ or argon gas, the ink solution was sprayed either directly onto Au electrodes or onto PVA-PDDA membranes. The aerosolized ink spray rapidly loses its solvent via evaporation upon reaching a surface and the dried PVA acts as a binder for the $\mathrm{Pt} / \mathrm{C}$ particles. In the case of spraying the ink onto PVA-PDDA membranes, the membrane needed to be repeatedly hydrated with $d \mathrm{H}_{2} \mathrm{O}$ in order to avoid cracking as the isopropanol and acetone in the ink mixture rapidly dried out the membranes while spraying. This PVA-PDDA membrane with $\mathrm{Pt} / \mathrm{C}$ catalyst bound to its surface was then hot pressed onto an $\mathrm{Au}$ electrode using a graphite epoxy. Optimal adhesion of the membrane to the electrode was achieved by using relatively high temperature (about $90^{\circ} \mathrm{C}$ ) and hotpressing the membrane 1 minute at a time. This was repeated three times, with light hydration of the membrane between presses. The results of this process are shown in Figure 32. 

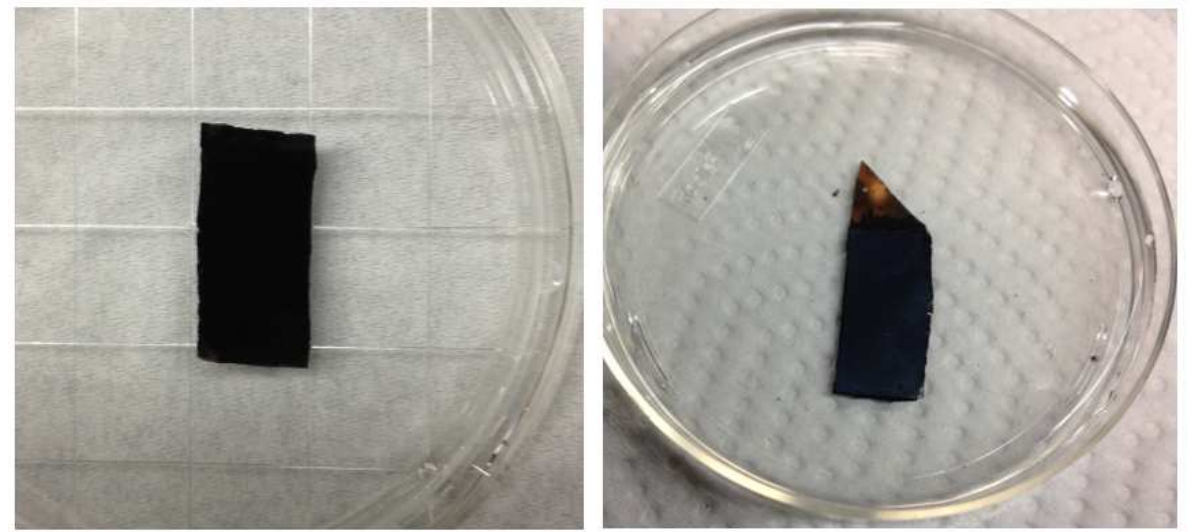

Figure 32: Image showing a PVA-PDDA membrane with Pt/C ink (left) and an Au electrode with PVAPDDA-Pt/C membrane epoxied onto its surface (right). Both images show the membrane or electrode submerged in $d \mathrm{H}_{2} \mathrm{O}$.

\subsection{Cyclic Voltammetry Analysis}

\subsubsection{Anode Cyclic Voltammetry Analysis}

CV analysis was performed using a BAS 100B Electrochemical Analyzer. A bare platinum wire was used as a counter electrode and an $\mathrm{Ag} / \mathrm{AgCl}$ electrode as the reference. Bovine heart cytochrome C (>95\% purity) was purchased from Sigma-Aldrich. The electrolyte solution used consisted of 10 mM Potassium Phosphate buffer (KPB), $500 \mathrm{mM} \mathrm{KCl}$ and $100 \mu \mathrm{M}$ of cytochrome C, with a $\mathrm{pH}$ of 7. The $\mathrm{KCl}$ is present to act as a background electrolyte, increasing the solution conductivity and thereby lowering the electrochemical systems internal resistance. Figures 33 and 34 show CV cycles of a $3 \mathrm{~cm}^{2} \mathrm{Au}-\mathrm{SAM}$ electrode in both the absence and presence of cytochrome $\mathrm{C}$. The cytochrome $\mathrm{C}$ free solution showed no anodic of cathodic peaks in the $0-100 \mathrm{mV}$ region where redox potential of the protein is expected. [25] The solution with cytochrome C present did however show a clear anodic peak at $86 \mathrm{mV}$ and a cathodic peak at $13 \mathrm{mV}$. Terrettaz et al. [25] showed anodic and cathodic peaks to be at $\sim 90$ and $\sim 10 \mathrm{mV}$ respectively, which is in line the results shown here. The CV scans were conducted multiple times, however without constant mixing the peak current would diminish and the voltage they appeared at would shift slowly towards $0 \mathrm{mV}$. 


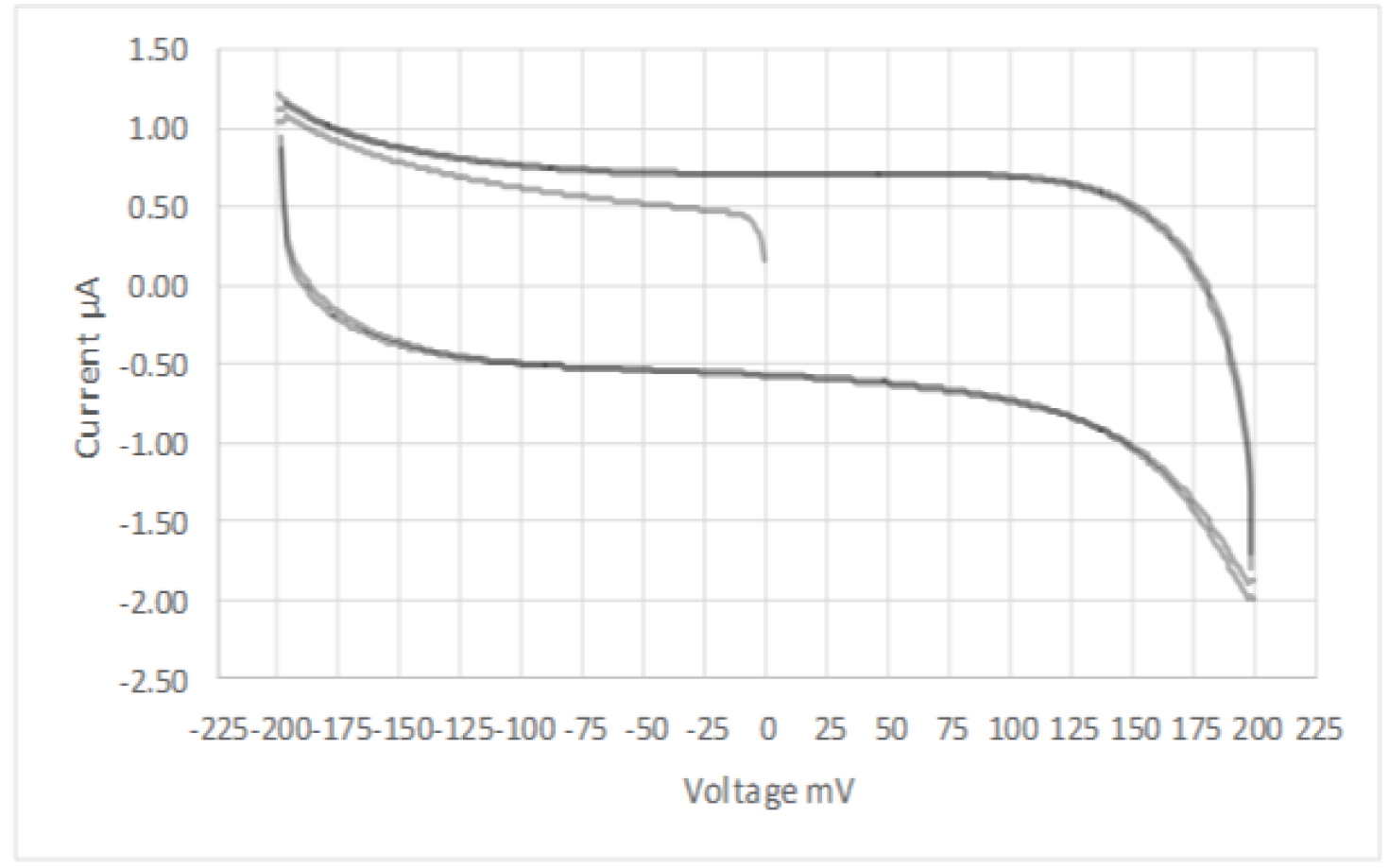

Figure 33: Image of CV scans of a $\omega$-Hydroxythiol Au-SAM electrode in a cytochrome C free solution. This scan was performed using a $25 \mathrm{mV} / \mathrm{s}$ scan rate and $1 \mu \mathrm{A} / \mathrm{V}$ sensitivity. No significant anodic or cathodic peaks were observed in the $0-100 \mathrm{mV}$ region.

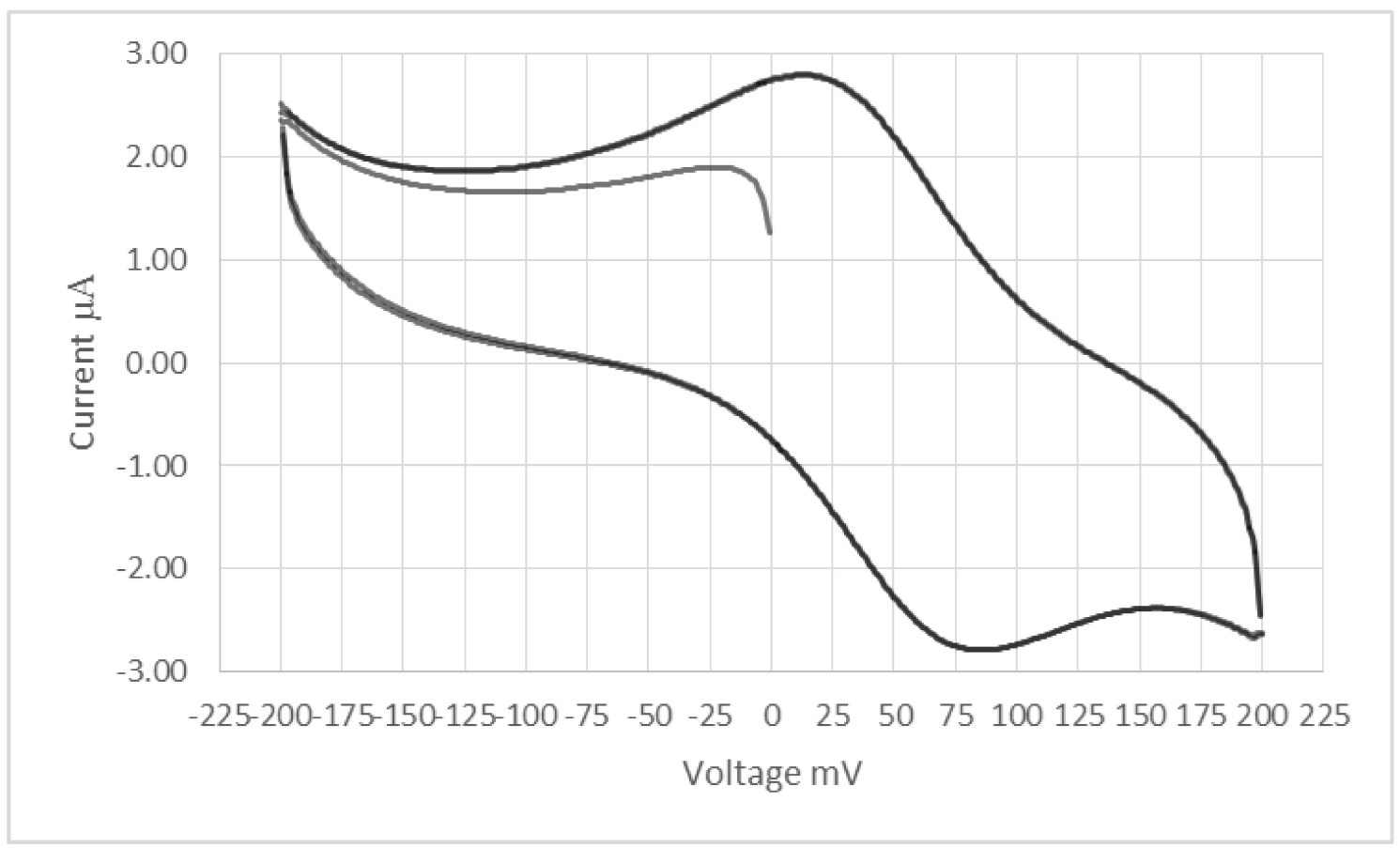

Figure 34: Image of $\mathrm{CV}$ scans of a $\omega$-Hydroxythiol Au-SAM electrode in a $5 \mu \mathrm{M}$ solution of cytochrome C. This scan was performed using a $25 \mathrm{mV} / \mathrm{s}$ scan rate and $1 \mu \mathrm{A} / \mathrm{V}$ sensitivity. The scan clearly showed strong cathodic and anodic peaks at $13 \mathrm{mV}$ and $86 \mathrm{mV}$, respectively. 


\subsubsection{Cathode Cyclic Voltammetry Analysis}

$\mathrm{CV}$ analysis was performed on $\mathrm{Au}-\mathrm{Pt} / \mathrm{C}$ cathodes and on cathodes coated with the PVA-PDDA anion exchange membrane. The same three electrode setup was used as for analyzing the anodes. The solution used contained $10 \mathrm{mM} \mathrm{KPB}, 100 \mathrm{mM} \mathrm{KCl}$ and was balanced to a $\mathrm{pH}$ of 7 . Figures 35 and 36 show $\mathrm{CV}$ scans for a $2 \mathrm{~cm}^{2}$ Au electrode with Pt/C catalyst using PVA as a binder. Figure 35 shows CV scans after deaerating the solution with vigorous purging using $N_{2}$ gas in order to remove $\mathrm{O}_{2}$ from the solution. The resulting scans showed that the current consumed by the oxygen reduction reaction at the cathode flattens out after $+300 \mathrm{mV}$ and does not show a significant cathodic reaction peak in the excepted range of $600-800 \mathrm{mV}$ with a current which stays under $1 \mathrm{~mA}$. Figure 36 shows CV scans of the same electrode with continuous bubbling of $\mathrm{O}_{2}$ gas. The scans clearly show an increase in current consumption as the voltage rises after $+300 \mathrm{mV}$, and a strong cathodic peak at $+700 \mathrm{mV}$ that exceeds $1.5 \mathrm{~mA}$. With the continuous addition of oxygen, the electrode had ample reactants for its oxygen reduction reaction, resulting in a high current consumption relative to the deaerated solution.

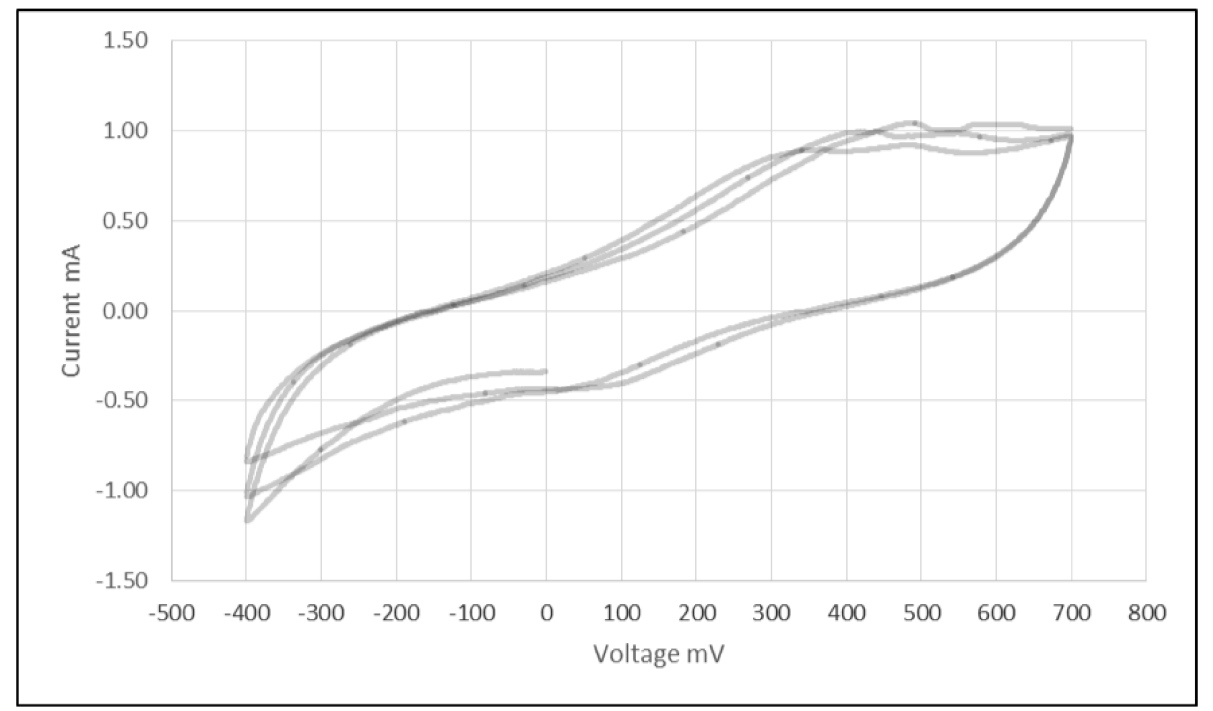

Figure 35: Image of CV scans of a $\mathrm{Pt} / \mathrm{C}$ on $\mathrm{Au}$ electrode in a deaerated buffer solution. This scan was performed at $10 \mathrm{mV} / \mathrm{s}$ scan rate and $1 \mathrm{~mA} / \mathrm{V}$ sensitivity. The current in this scan showed a clear flattening in the $+300 \mathrm{mV}$ region, where an rise in current would be expected if oxygen reduction was taking place. 


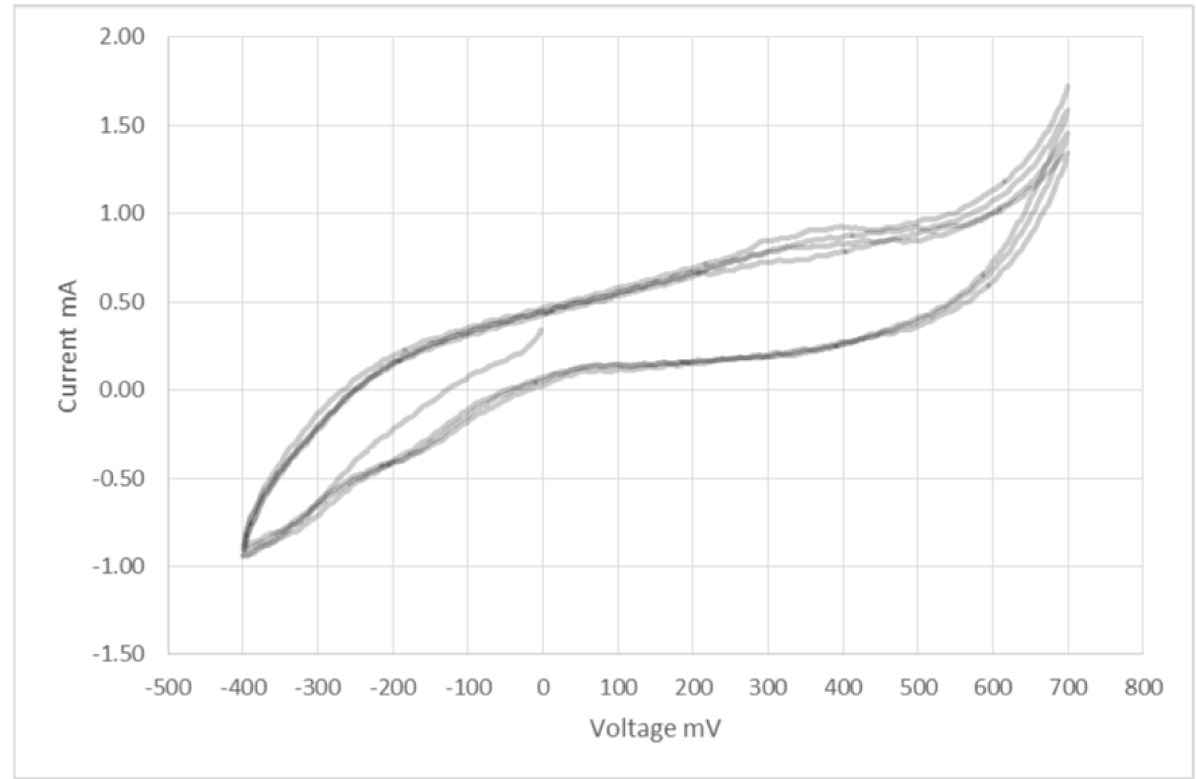

Figure 36: Image of $\mathrm{CV}$ scans of a $\mathrm{Pt} / \mathrm{C}$ on $\mathrm{Au}$ electrode in a oxygen rich buffer solution. This scan was performed at a $10 \mathrm{mV} / \mathrm{s}$ scan rate and $1 \mathrm{~mA} / \mathrm{V}$ sensitivity. The current in this scan clearly showed a signicant increase in current in the $+300 \mathrm{mV}$ region, indicating oxygen reduction was taking place.

Figures 37 and 38 show $\mathrm{CV}$ scans for a $1.5 \mathrm{~cm}^{2}$ Au electrode with a Pt/C catalyst bound to a PVA-PDDA anion exchange membrane. The same buffer solution and three electrode protocol was used as before. As with the membrane free electrodes, scans were performed in deaerated and oxygen rich solutions. Figure 37 shows the CV scans of the membrane bound electrode in an oxygen depleted solution. For this particular CV scan, the scan rate was lowered and the sensitivity increased in order to try to display any significant cathodic or anodic peaks that may be lost using standard settings. The result of this scan showed diminished and erratic positive current in the + 300-700 $\mathrm{mV}$ region, with the maximum current never reaching more than $0.4 \mathrm{~mA}$. This result was very low compared to the membrane free cathode scans. The most likely explanation is that the cathode is scavenging the oxygen left inside the anion exchange membrane, leading to decreased and irregular current.

Figure 38 shows the CV scans of the membrane bound cathode in an oxygen rich solution. Unlike the deaerated solution scans, these results were much more in line with scans of membrane free cathodes, showing a clear increase in current consumption after $+300 \mathrm{mV}$ with a strong cathodic peak in the range of $+700-800 \mathrm{mV}$. The current density of the anion exchange membrane bound cathode was on par with that of the membrane free cathode, indicating that oxygen 


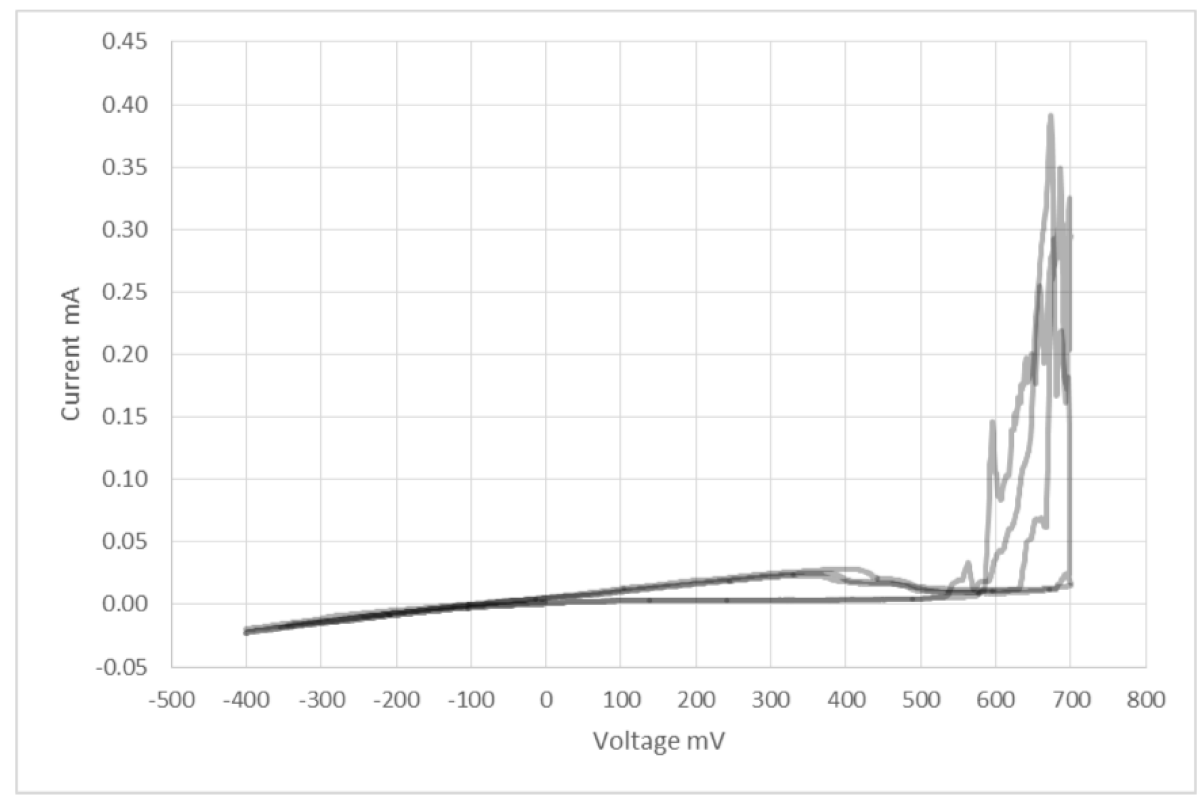

Figure 37: Image of $\mathrm{CV}$ scans of a $\mathrm{Pt} / \mathrm{C}$ anion exchange membrane $\mathrm{Au}$ electrode in a deaerated buffer solution. This scan was performed at a $25 \mathrm{mV} / \mathrm{s}$ scan rate and $100 \mu \mathrm{A} / \mathrm{V}$ sensitivity. Relative to AEM free cathodes, this scan showed an extremely low current density throughout the entire voltage range. The $+300 \mathrm{mV}$ region showed an increase in current, however the current is irregular and even at its highest values was still less than $50 \%$ of the current observed in $\mathrm{AEM}$ free $\mathrm{Pt} / \mathrm{C}$ cathodes.

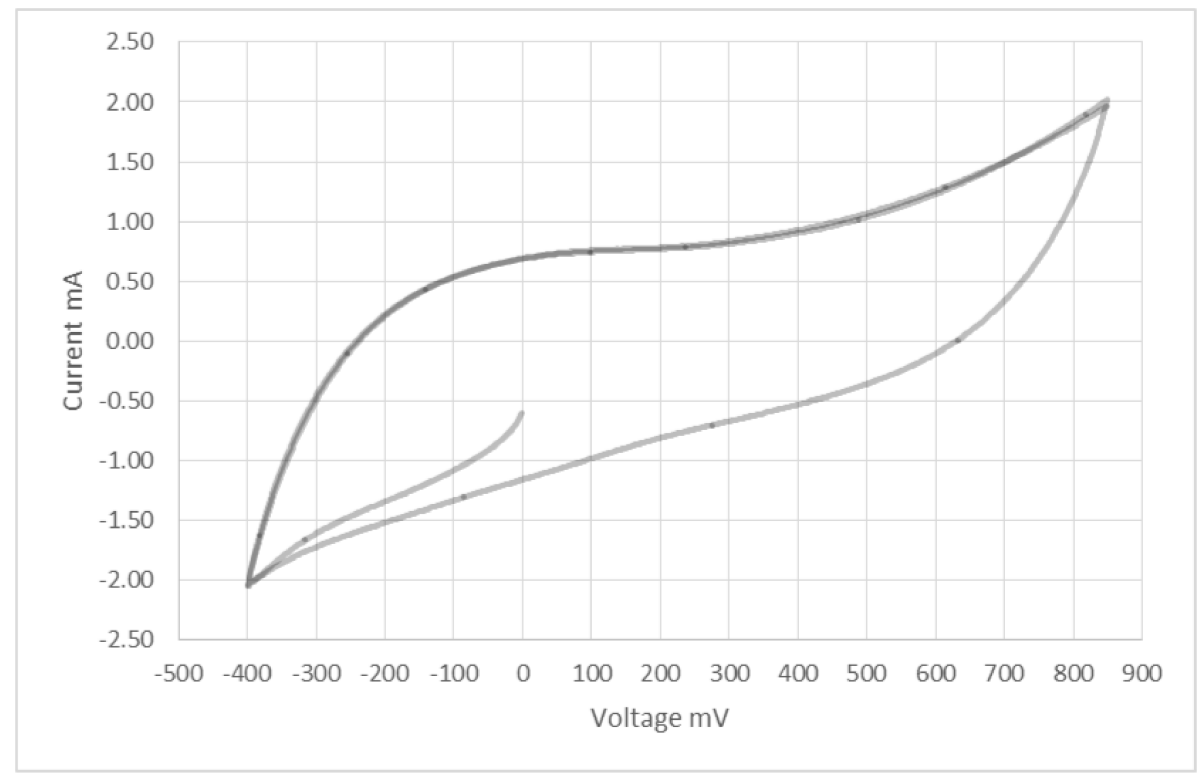

Figure 38: Image of $\mathrm{CV}$ scans of a $\mathrm{Pt} / \mathrm{C}$ anion exchange membrane $\mathrm{Au}$ electrode in a oxygen rich buffer solution. The scan was performed at a $50 \mathrm{mV} / \mathrm{s}$ scan rate and $1 \mathrm{~mA} / \mathrm{V}$ sensitivity. The current in this scan clearly showed a signicant increase in current in the $+300 \mathrm{mV}$ region, indicating oxygen reduction was taking place. 
permeability was not a limiting factor with this type of membrane. These results clearly show that the $\mathrm{Pt} / \mathrm{C}$ cathode constructed here was capable of catalyzing an oxygen reduction reaction at a current density exceeding $750 \mu \mathrm{A} / \mathrm{cm}^{2}$, which is more than sufficient for a photoelectrochemical cell of this design.

\subsubsection{Conclusion}

The cyclic voltammetry results showed that both the anode and cathode were functioning as expected. The Au-SAM anode, when cytochrome $\mathrm{C}$ was present, showed clear anodic and cathodic peaks in line with expected results. This showed that the anode was capable of accepting electrons from reduced cytochrome $\mathrm{C}$ in solution. The $\mathrm{CV}$ results from the cathode, both with and without the anion exchange membrane showed very simular results in the presence of oxygen. Both cathodes showed high fidelity in the shape of their respective curves and in the current densities observed in the $+300 \mathrm{mV}$ region. The high current density of the $\mathrm{Pt} / \mathrm{C}$ cathodes with anion exchange membranes in the presence of oxygen show that oxygen permeability is not an issue in such a system. Finally, high current density of these $\mathrm{Pt} / \mathrm{C}$ cathodes $\left(>750 \mu \mathrm{A} / \mathrm{cm}^{2}\right.$ in the ORR region) relative to the anode (roughly $1 \mu \mathrm{A} / \mathrm{cm}^{2}$ at the anodic peak) indicated that the oxidation of cytochrome $\mathrm{C}$ and not the ORR would be the limiting reaction step in in the photocell.

\subsection{Plastoquinone and Cytochrome $\mathrm{C}$ redox Activity}

Before a working photoelectrochemical cell could be produced, it was first necessary to confirm that plastoquinone and cytochrome $\mathrm{C}$ are in fact capable of redox reactions with one another. This was tested by producing a solution of reduced PQ (plastoquinol) dissolved in a lipids as outlined in Kruk et. al. [20] Special care had to be taken as once plastoquinol is produced, it will quickly be oxidized back to plastoquinone with any exposure to oxygen in the air. First a deaerated solution of ethanol was used to produce a solution of PQ at a concentration of $5 \mathrm{mg} / \mathrm{mL}$. Sodium borohydride was then added to the solution along with a small amount of dilute $\mathrm{HCl}$ to maintain an acidic pH. After filtering out any excess $\mathrm{NaBH}_{4}$ by filtering the solution through cotton, three volumes of a $1: 1$ mixture of hexane and $d \mathrm{H}_{2} \mathrm{O}$ were added to the solution to pull the PQ out 
of the ethanol and extract PQ into an organic phase. The hexane had been heavy deaerated, and the water had been treated with sodium dithionite to remove any oxygen that might have been present. To this hexane-PQ mixture, extracted lipids dissolved in hexane were added to produce a PQ to lipid ratio of $1: 25$ by mass. The hexane from this lipid-PQ mixture was then removed via rotary evaporation. The lipid-PQ mixture was than dissolved in a minimal amount of ethanol and injected into a deaerated buffer solution containing $50 \mathrm{mM}$ HEPES, $20 \mathrm{mM} \mathrm{NaCl}, 5 \mathrm{mM} \mathrm{MgCl} 2$ and $5 \mu \mathrm{M}$ cytochrome C. The final concentration of the lipids in this solution was $433 \mu \mathrm{g} / \mathrm{mL}$. This solution was then immediately analyzed using spectroscopy to observe cytochrome $\mathrm{C}$ reduction.

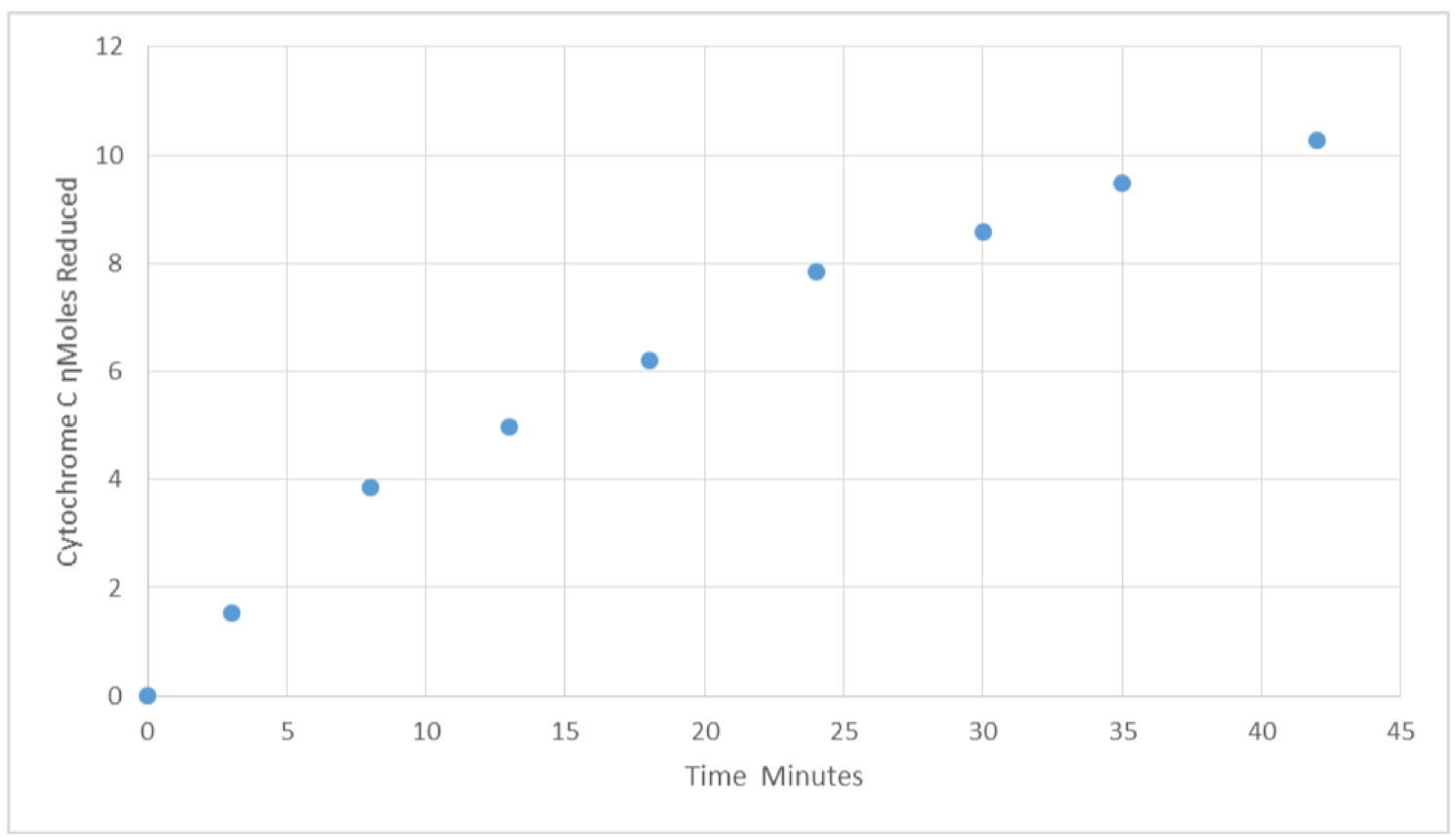

Figure 39: Graph of cytochrome $\mathrm{C}$ reduction by plastoquinol versus time. Reduction of cytochrome $\mathrm{C}$ was observed spectroscopically, in a solution of $5 \mu \mathrm{M}$ cytochrome $\mathrm{C}$ and $17 \mu \mathrm{g} / \mathrm{mL}$ plastoquinol.

Reduction of cytochrome C can be observed spectroscopically, the absorbance of oxidized Cc at $550 \mathrm{~nm}$ falls as it is reduced. [63] Provided that plastoquinol is handled carefully in its reduced form and that it is added immediately to the solution, we can easily observe the reduction of cytochrome C by plastoquinol. The results of this test are pictured in Figure 39. As expected, the rate of reduction is very high in the first few minutes, over 0.5 nMoles per minute, however by 30 minutes this reduction rate has dropped by almost $50 \%$ as the reduced $\mathrm{Cc}$ is saturated and the concentration plastoquinol drops. 


\section{Photoelectrochemical Cell Assembly and Testing}

The photoelectrochemical cell was made in a 1 x $3 \mathrm{~cm}$ plastic cuvette typically used for UV-Vis spectroscopy. The anode and cathode were placed on opposite edges of the cuvette along its opaque sides, to allow light to shine through the cell. The electrodes were held in place using crocodile clips, which connected the system to HP 4145B semiconductor parameter analyzer (SPA) to test the amount of photocurrent that could be generated. A $50 \mathrm{~W}$ halogen lamp was used as a light source, the distance to the photocell was calibrated to illuminate the device with one sun's worth of light energy using a IXOLAR SolarBit photovoltaic cell. This setup allows for solutions of varying concentration of running solutions to be added and taken out without disassembling the entire device. The entire apparatus was placed on top of a magnetic stir plate and a small stirring bar was present at the bottom of the cuvette to provide constant but light stirring. Figure 40 illustrates the design of this photoelectrochemical cell.

To house PSII and plastoquinone, liposomes of the extract lipids were produced using following simple method laid out in Akbarzadeh et. al. 2013. [42] The desired mass of lipids and plastoquinone were dissolved into a $1: 1$ solution of chloroform : methanol and then dried using rotary evaporation in small glass vials. Lipids form a film along the sides of the glass vial during the evaporation process, after which a buffered solution was added, and the glass vial was sonicated using a water bath for 20 minutes to produce a uniform mixture of liposomes. PSII was incorporated into the liposomes simply by injected a concentrated solution of PSII into a liposome solution and incubating the mixture for 20 minutes. It has been shown that PSII proteins will spontaneously integrate themselves into liposomes with little to no free protein in solution, provided the ratio of chlorophyll to lipids is favorable. [43] The 'standard' running solution used was as follows; 20 mM HEPES buffer, $5 \mathrm{mM} \mathrm{MgCl}_{2}, 100 \mathrm{mM} \mathrm{KCl}$ and $400 \mathrm{mM}$ sucrose balanced to $\mathrm{pH} 7$ served as the buffer solution. Lipids were used at a final concentration of $443 \mu \mathrm{g} / \mathrm{mL}$ with a ratio of 2 : $1: 1$ for MGDG, DGDG and SQDG respectively. This ratio of negatively charged and HII phase lipids emulates that found in the thylakoid membrane. Plastoquinone was added to the liposome mixture at a mass ratio of $15: 1$, lipid to plastoquinone. PSII was used at a final concentration of $40 \mu \mathrm{g} / \mathrm{mL}$ chlorophyll, this produces the desired lipid to chlorophyll ratio of 


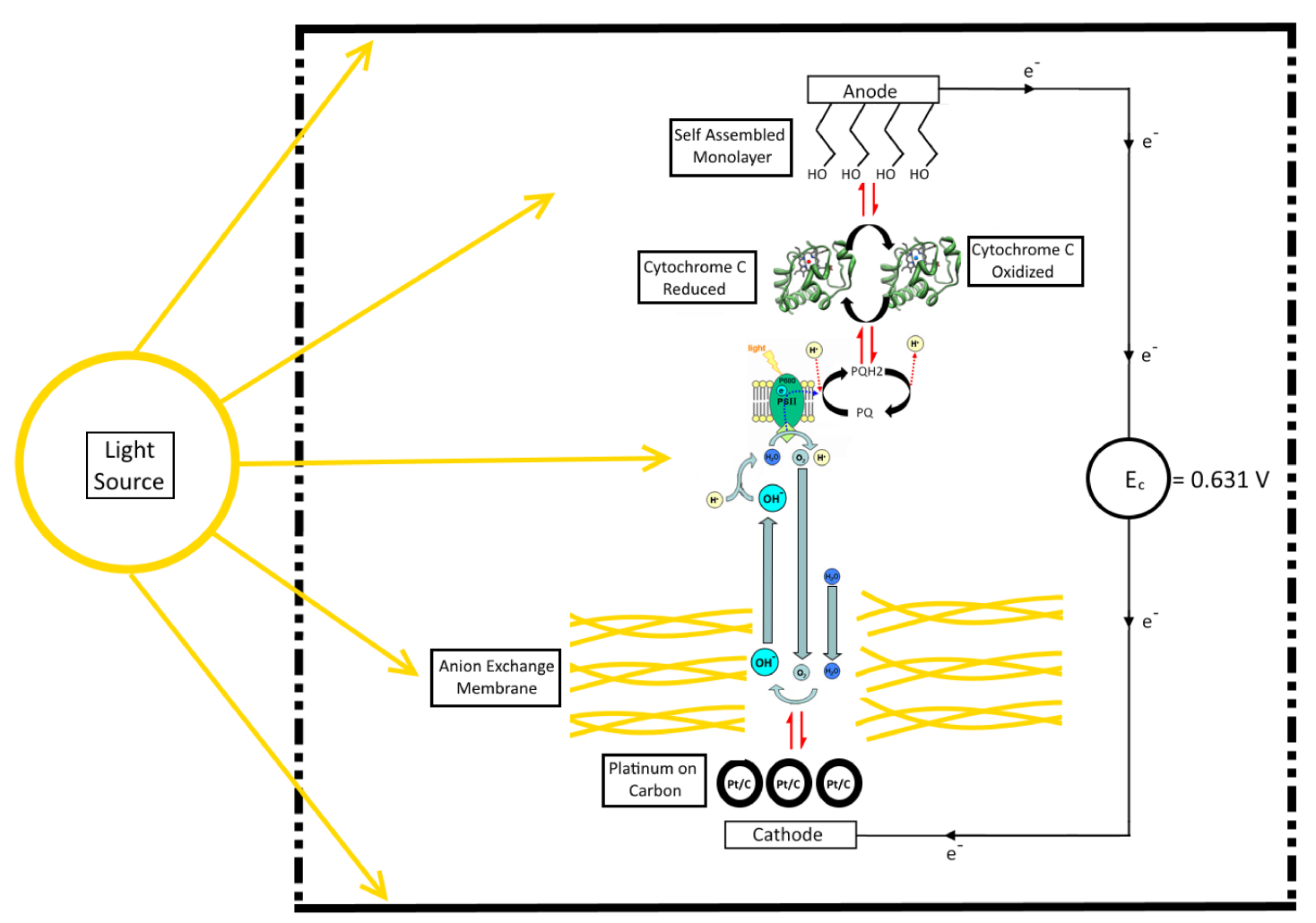

Figure 40: Diagram of the Photosystem II photoelectrochemical cell with all components present. The entire system is inside a cuvette, shown as the square surrounding the PEC components. The dashed lines represent the transparent sides of the cuvette while the solid lines represent the opaque sides. The electrodes are placed along the opaque sides of the cuvette, allowing light to travel through the entire cell in order to start the reaction.

$10: 1$. When thylakoid membrane extracts were used in the system, the same final concentration of chlorophyll, $40 \mu \mathrm{g} / \mathrm{mL}$, was used as well. Cytochrome $\mathrm{C}$ was used at a final concentration of 5 $\mu \mathrm{M}$. The electrodes present in this system both had an active surface of $1 \mathrm{~cm}^{2}$. Prior to scanning the photocell with the SPA, the system was sealed using parafilm and no $\mathrm{O}_{2}$ was bubbled into the system. The idea being that PSII oxygen evolution should produce $\mathrm{O}_{2}$ for the device as it is illuminated with light.

The SPA was used to determine the amount of current generated over time by the system when a potential was placed across the two electrodes. Since the photosynthetic proteins were not directly electrically connect to the anode, it was necessary to test the photocurrent generated over 
time in order to see where the current would remain constant. Generally the current would be quite high at first and then begin to drop, and after about 1-2 minutes the signal from the current would stabilize. The first step was to find an appropriate voltage with which to run the system at. This was done using a solution of thylakoid membranes instead of PSII-liposomes. Based on the results from Kruk et. al. 2003 [19] it is known that cytochrome C can be reduced directly by thylakoid membranes, it was also found that thylakoid membranes when prepared freshly do not adhere to the anion exchange membrane surface as much as the PSII-liposomes and therefore produce more consistent photocurrents. The effect of PSII-liposome adhesion to the anion exchange membrane can be mitigated by sonication of PSII solution and $\mathrm{KOH}$ treatment of the cathode. However, for determining the running cell voltage thylakoid solutions proved more consistent results when photocurrent tests were performed repeatedly at different cell voltages. In order to eliminate interference from background current from species in the solution, the current was found during dark and light conditions, with the values listed in tables 2 and 3 being the difference between dark and light current.

\subsection{Photoelectrochemical Cell Results}

Figure 41 shows I/V sweeps in buffer only and a standard PEC mixture in light and dark conditions. The current shown in these figures is positive current flow from the anode into the cathode, and at about $750 \mathrm{mV}$ there was a large difference in the generated current between the light and dark exposed PEC mixtures, about $300 \mu \mathrm{A}$. Figure 42 shows current generated by a 'standard' PEC mixture under light and dark conditions when the cell potential was held at $750 \mathrm{mV}$. Clearly the actual photocurrent that can be produced over an extended period of time was much smaller (about $9 \mu \mathrm{A})$ than the I/V sweeps would indicate.

As seen in Table 2, it was found that $750 \mathrm{mV}$ was a suitable operating cell voltage as lower voltages produced a much lower photocurrent and voltages above $750 \mathrm{mV}$ produced diminishing returns on the generated photocurrent. Table 3 shows the photocurrent generated under differing conditions, testing different concentration of PSII, cytochrome $\mathrm{C}$ and plastoquinone, as well as different proportions of lipids to see how the photocurrent is affected. 


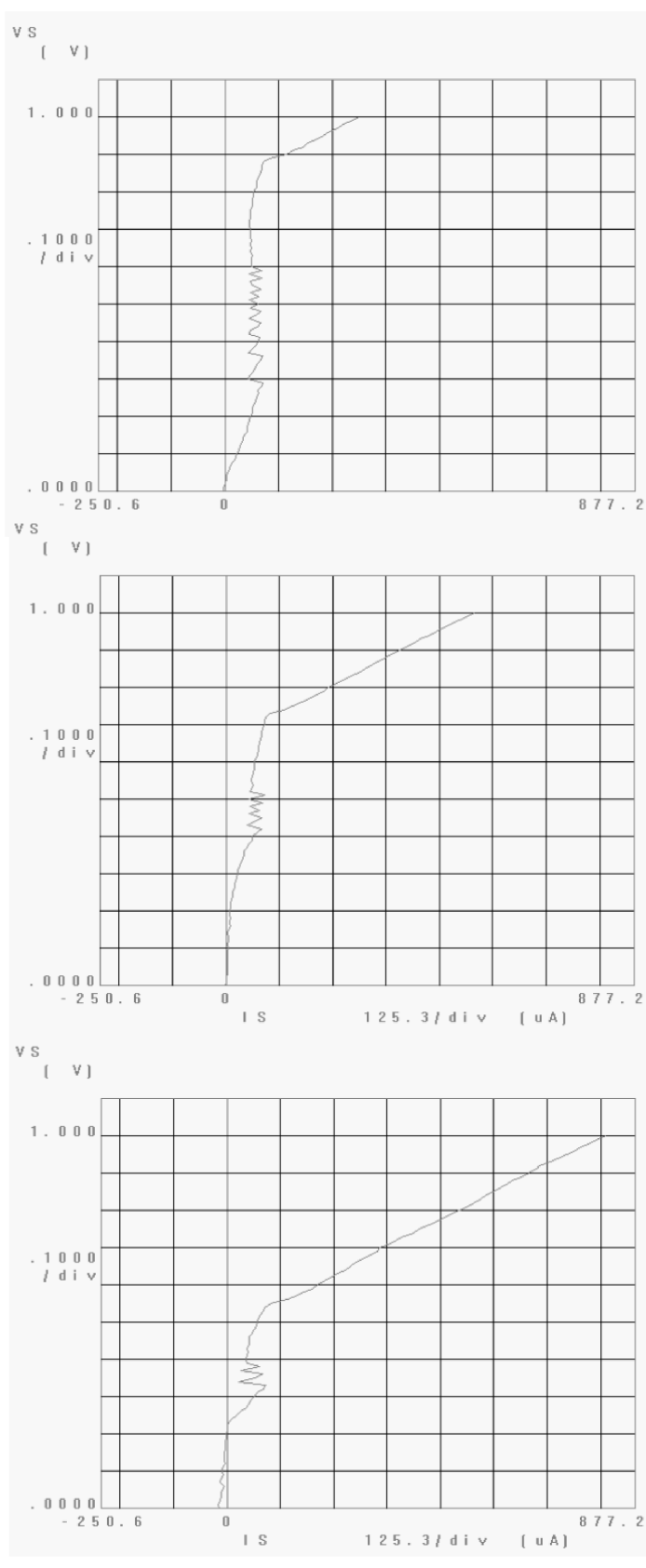

Figure 41: Shows I/V curves of photoelectrochemical cells running 'standard' conditions. Includes a buffer only solution (top), 'standard' PEC mixture under dark (center) and 'standard' PEC mixture under light (bottom) conditions.
Increasing the concentration of PSII had no effect on the photocurrent generated, however decreasing the concentration by half resulted in a greater than $50 \%$ drop in photocurrent. The 'standard' concentration of $40 \mu \mathrm{g} / \mathrm{mL}$ was chosen as the absorbance at $680 \mathrm{~nm}$ in a solution of PSII at $40 \mu \mathrm{g} / \mathrm{mL}$ is $99 \%$. $680 \mathrm{~nm}$ is the absorbance of the primary electron donor in PSII, and was therefore a suitable wavelength to base the concentration of PSII on. Since raising PSII concentration had no effect but lowering it drastically lowers photocurrent, clearly PSII concentration is not the limiting step in the photocells electrochemical cycle when a concentration of 40 $\mu \mathrm{g} / \mathrm{mL}$ is used.

Altering the concentration of cytochrome C concentration had more predictable effects, when increased the photocurrent saw a small but noticeable increase, and when lowered to one fifth its original concentration the photocurrent dropped by over $50 \%$. Given the low current density of the anode in $\mathrm{CV}$ analysis relative to the cathode, it was thought that the system would be highly dependent on cytochrome $\mathrm{C}$ concentration. When both cytochrome C and PSII / thylakoid concentrations were increased, photocurrent did increase as well, but only marginally in the case of PSII. Cytochrome C is known to cause aggregation in liposome solutions at high concentrations, and it is possible that with a lower chlorophyll to lipid ratio that aggregation could be more pronounced leading to sluggish diffusion of 
cytochrome $\mathrm{C}$, lowering the electron transfer rate.

Changes in the proportion of plastoquinone to lipids had very interesting effects on the photocurrent, increasing the PQ : lipid ratio lowered the photocurrent while decreasing the ratio increased the photocurrent. While unintuitive, these results are not without merit. As found in Kruk et. al. 1992 [20] the highest activity of PQ has been found in membrane systems where the PQ : lipid ratio was near the $1: 140$ ratio found in thylakoid membranes as the proportion of PQ in the membrane can affect the membrane properties. In particular, PQ is known to be localized near the surface of lipid membranes when in low PQ : lipid ratios, with higher ratios burying the PQ deeper in the membrane. This explains the drop in photocurrent, as PQ-Cc redox activity is diminished in high $\mathrm{PQ}$ : lipid ratios. The increase in photocurrent with lower PQ : lipid ratios is also in line the findings in Kruk et. al. 2003 [19], where they found the highest rates of cytochrome $\mathrm{C}$ reduction were found in liposomes containing the lowest proportions of $\mathrm{PQ}$.
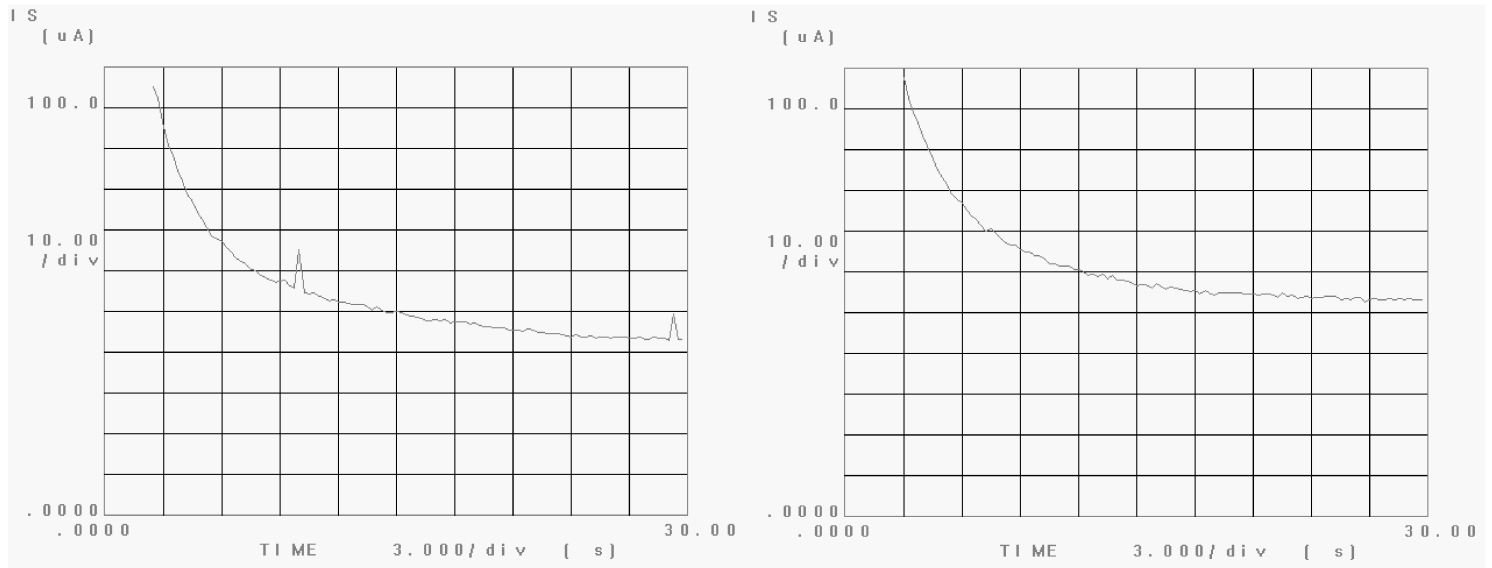

Figure 42: Current produced by a 'standard' PEC mixture under dark (left) and light (right) conditions at a constant cell potential of $750 \mathrm{mV}$.

Altering the ratios of lipids had drastic negative effects on the photocurrent of the system. When SQDG was removed from the liposome composition, as predicted, the photocurrent of the system dropped dramatically. This is in line with work by Kruk and associates, showing that lipids with negative head groups are key for PQ - cytochrome $\mathrm{C}$ redox activity. It might follow that by increasing the proportion of SQDG, the redox activity between PQ and cytochrome C could be enhanced. However as evidenced in our results, increasing the proportion of SQDG and making it $50 \%$ of the lipid composition by mass, produced the lowest photocurrent out of all our 


\begin{tabular}{|c|c|}
\hline Voltage $\mathrm{mV}$ & Current $\mu \mathrm{A}$ \\
\hline 500 & 4.5 \\
\hline 650 & 5.0 \\
\hline 750 & 9.0 \\
\hline 850 & 10.5 \\
\hline
\end{tabular}

Table 2: Photocurrent generated by thylakoid PEC under 'standard' conditions at varying cell potentials.

\begin{tabular}{|c|c|}
\hline Conditions & Current $\mu \mathrm{A}$ \\
\hline 'Standard' & 9.0 \\
\hline High PSII $(60 \mu \mathrm{g} / \mathrm{mL})$ & 9.0 \\
\hline Low PSII $(20 \mu \mathrm{g} / \mathrm{mL})$ & 4.0 \\
\hline High Cc $(10 \mu \mathrm{M})$ & 10.5 \\
\hline Low Cc $(1 \mu \mathrm{M})$ & 3.0 \\
\hline High PQ (1:5) & 2.5 \\
\hline Low PQ $(1: 50)$ & 11.5 \\
\hline $\begin{array}{c}\text { High Cc }(10 \mu \mathrm{M}) \& \\
\text { High PSII }(60 \mu \mathrm{g} / \mathrm{mL})\end{array}$ & 9.5 \\
\hline $\begin{array}{l}\text { High Cc }(10 \mu \mathrm{M}) \& \\
\text { High Thy. }(60 \mu \mathrm{g} / \mathrm{mL})\end{array}$ & 10.0 \\
\hline $\begin{array}{l}\text { MG : DG : SQ } \\
(1: 1: 2)\end{array}$ & 2.0 \\
\hline $\begin{array}{c}\mathrm{MG}: \mathrm{DG}: \mathrm{SQ} \\
(1: 1: 0)\end{array}$ & 2.5 \\
\hline
\end{tabular}

Table 3: Photocurrent generated under 'standard' and other varying conditions at cell potential of $750 \mathrm{mV}$. 
results. The drop in photocurrent is likely due to the lower amount of MGDG, which can form the reverse micelles needed for cytochrome $\mathrm{C}$ and $\mathrm{PQ}$ to interact. A more innate reason for this drop in activity could be that by altering the proportions of HII and lamellar phase lipids, the morphology of the liposome surface was changed in such a way that potential PQ - cytochrome C interactions were inhibited.

In regards to the systems longevity, it was found that the PSII-liposomes were much less reliable than pure thyalkoid membranes. Even with pretreatment of the AEM with $\mathrm{KOH}$ and heavy sonication of PSII solutions, it was still found that after approximately 30 minuntes most of the liposomes would aggregate onto the AEM surface. This resulted in little to no photocurrent being generated. However when using thyalkoid membranes, provided they were used soon after extraction without a freeze-thaw cycle, would not aggergate onto the AEM. Even after 1 hour of testing the photocurrent of a thyaloid-PEC did not diminish significantly.

\subsection{Conclusion}

This body of work would appear to show with some certainty that a photoelectrochemical device using a PSII-PQ-Cc electron transport chain to capture solar energy can function. However it is also shown that the current generated, while on the same order of magnitude as other such devices as seen in Table 4, was extremely small. Combined with the lack of any significant difference in open circuit voltage and short circuit current under light and dark conditions, the feasibility of such a device in its current iteration is suspect at best.

The most likely reason for the lack of significant Voc and Isc under light and dark conditions is due to the decoupled nature of the photocell, in particular at the anode. In most bio-photocells the photosynthetic proteins that acts as the source of electrons for the circuit are immobilized directly onto the surface of the anode [35] [37] [39] [40], this is not the case in this body of work. The liposomes that contains PSII and cytochrome $\mathrm{C}$ are freely floating in the solution, meaning that the amount of current that can be generated will be limited by not only the electron transfer kinetics at the anode but also by the diffusion of cytochrome $\mathrm{C}$ throughout the cell. As seen in Table 3 changing the concentration of cytochrome $\mathrm{C}$ had a significant effect on the 
photocurrent generated, clearly showing that the photocurrent was highly limited by the amount of reduced cytochrome $\mathrm{C}$ that can reach the anode.

It would appear that by trying to provide a more physiologically compatible environment for PSII resulted in a lack of Voc and Isc as well as lower photocurrent generation. Moving forward with such research it will be necessary to see how the current density of this reaction can be enhanced, possibly by integrating an enzyme such as cytochrome $\mathrm{C}$ oxidase into the anodes surface. Another area of interest would be to investigate the use of thylakoid membranes instead of PSII-liposomes as the primary method for housing photosynthetic proteins in such a device.

\begin{tabular}{|c|c|c|}
\hline Photocell Description & Maximum & Reference \\
\hline Current Density & \\
\hline TiOn dye sensitized solar & $1,000 \mu \mathrm{A} / \mathrm{cm}^{2}$ & {$[37]$} \\
cell using PSI & $600 \mu \mathrm{A} / \mathrm{cm}^{2}$ & {$[35]$} \\
\hline Immobilized PSII based & & {$[39]$} \\
\hline photoelectrochemical cell & & {$[40]$} \\
\hline Immobilized thyalkoid based & $68 \mu \mathrm{A} / \mathrm{cm}^{2}$ & This body \\
photoelectrochemical cell & & of work \\
\hline PSII liposome based & $11.5 \mu \mathrm{A} / \mathrm{cm}^{2}$ & \\
\hline photoelectrochemical cell & & \\
\hline
\end{tabular}

Table 4: Shows the maximum current density of various bio-photocell devices. Note that current reported for the PSII liposome based photoelectrochemical cell is using photocurrent generated under the applied potential of $+750 \mathrm{mV}$. 


\section{References}

[1] IEA, Electricity Information 201\%. Internation Energy Agency, 2017.

[2] D. R. Dunn, Monthly Energy Review November 2017, pp. 1-28. U.S. Energy Information Administration, 2017.

[3] V. Smil, Transforming the Twentieth Century: Technical Innovations and Their Consequences. Oxford University Press, 2nd ed., 2006.

[4] S. Sharma, K. Jain, and A. Sharma, "Solar cells: In research and applications - a review," Materials Sciences and Applications, vol. 8, pp. 1145-1155.

[5] C. Honsberg and S. Bowden, "Solar cell operation - solar cell structure," 2017.

[6] E. W. McFarland, "Solar energy: setting the economic bar from the top-down," Energy Environ. Sci., vol. 7, pp. 846-854, 2014.

[7] F. R. F. D. M. R. W. M. and A. K., U.S. Solar Photovoltaic System Cost Benchmark: Q1 2017. National Renewable Energy Laboratory, 2017.

[8] N. A. Campbell and J. B. Reece, Biology. Benjamin Cummings, 8th ed., 2008.

[9] G. Renger, "Chapter 1 overview of primary processes of photosynthesis," in Primary Processes of Photosynthesis, Part 1: Principles and Apparatus, vol. 8, pp. 5-35, The Royal Society of Chemistry, 2008.

[10] K. P. E. Atwell B. J. and T. C. G. N., Plants in Action. Macmillan Education Australia, 1999.

[11] B. Hankamer, , J. Barber, and E. J. Boekema, "Structure and membrane organization of photosystem II in green plants," Annual Review of Plant Physiology and Plant Molecular Biology, vol. 48, no. 1, pp. 641-671, 1997. PMID: 15012277.

[12] A. Zouni, "Chapter 15 from cell growth to the 3.0 a resolution crystal structure of cyanobacterial photosystem II," in Primary Processes of Photosynthesis, Part 2: Principles and Apparatus, vol. 9, pp. 193-236, The Royal Society of Chemistry, 2008.

[13] N. A. Eckardt, "A role for PsbZ in the core complex of photosystem II," The Plant Cell, vol. 13, no. 6, pp. 1245-1248, 2001. 
[14] G. Renger, "Chapter 16 functional pattern of photosystem II," in Primary Processes of Photosynthesis, Part 2: Principles and Apparatus, vol. 9, pp. 237-290, The Royal Society of Chemistry, 2008.

[15] G. W. Bushnell, G. V. Louie, and G. D. Brayer, "High-resolution three-dimensional structure of horse heart cytochrome C " J. Mol. Biol., vol. 214, pp. 585-595, Jul 1990.

[16] M. Huttemann, P. Pecina, M. Rainbolt, T. H. Sanderson, V. E. Kagan, L. Samavati, J. W. Doan, and I. Lee, "The multiple functions of cytochrome $\mathrm{C}$ and their regulation in life and death decisions of the mammalian cell: From respiration to apoptosis," Mitochondrion, vol. 11, pp. 369-381, May 2011.

[17] J. B. Neilands, "The isolation and properties of cytochrome C from different sources," J. Biol. Chem., vol. 197, pp. 701-708, May 1952.

[18] C. Kawai, F. M. Prado, G. L. Nunes, P. Di Mascio, A. M. Carmona-Ribeiro, and I. L. Nantes, " $\mathrm{pH}-$ Dependent interaction of cytochrome $\mathrm{C}$ with mitochondrial mimetic membranes: the role of an array of positively charged amino acids," J. Biol. Chem., vol. 280, pp. 34709-34717, Oct 2005.

[19] J. Kruk, M. JemioLa-RzemiNska, and K. StrzaLka, "Cytochrome c is reduced mainly by plastoquinol and not by superoxide in thylakoid membranes at low and medium light intensities: its specific interaction with thylakoid membrane lipids," Biochem. J., vol. 375, pp. 215-220, Oct 2003.

[20] J. Kruk, K. Strza?ka, and R. M. Leblanc, "Monolayer study of plastoquinones, alphatocopherol quinone, their hydroquinone forms and their interaction with monogalactosyldiacylglycerol. Charge-transfer complexes in a mixed monolayer," Biochim. Biophys. Acta, vol. 1112, pp. 19-26, Nov 1992.

[21] B. de Kruijff and P. Cullis, "Cytochrome C specifically induces non-bilayer structures in cardiolipin-containing model membranes," Biochimica et Biophysica Acta (BBA) - Biomembranes, vol. 602, no. 3, pp. $477-490,1980$. 
[22] M. Fedurco, "Redox reactions of heme-containing metalloproteins: dynamic effects of selfassembled monolayers on thermodynamics and kinetics of cytochrome c electron-transfer reactions," Coordination Chemistry Reviews, vol. 209, no. 1, pp. 263 - 331, 2000.

[23] A. SzUcs and M. NovAk, "Stable and reversible electrochemistry of cytochrome c on bare electrodes part 1. effect of ionic strength," Journal of Electroanalytical Chemistry, vol. 383, no. 1 , pp. $75-84,1995$.

[24] E. Pensa, E. Cortes, G. Corthey, P. Carro, C. Vericat, M. H. Fonticelli, G. Benitez, A. A. Rubert, and R. C. Salvarezza, "The chemistry of the sulfur-gold interface: in search of a unified model," Acc. Chem. Res., vol. 45, pp. 1183-1192, Aug 2012.

[25] S. Terrettaz, J. Cheng, C. J. Miller, and R. D. Guiles, "Kinetic parameters for cytochrome C via insulated electrode voltammetry," Journal of the American Chemical Society, vol. 118, no. 33, pp. $7857-7858,1996$.

[26] Grutter, "Microcantilver-based sensors, thiol expt.." McGill SPM Group.

[27] P. A. Seigenthaler and N. Murata, Lipids in Photosynthesis: Structure, Function and Genetics. Kluwer Academic Publishers, 1998.

[28] J. Jouhet, "Importance of the hexagonal lipid phase in biological membrane organization," Front Plant Sci, vol. 4, p. 494, 2013.

[29] D. C. Harris, Quantitative Chemical Analysis. W H Freeman \& Co, 7th ed., 2006.

[30] W. C. Still, M. Kahn, and A. Mitra, "Rapid chromatographic technique for preparative separations with moderate resolution," The Journal of Organic Chemistry, vol. 43, no. 14, pp. 2923$2925,1978$.

[31] C. Song and J. Zhang, PEM Fuel Cell Electrocatalysts and Catalyst Layers, ch. Electrocatalytic Oxygen Reduction Reaction, pp. 89-134. Springer, London, 2008.

[32] M.-P.-I. für Eisenforschung, "Understanding the mechanism of the oxygen reduction reaction on Pt," 2017.

[33] A. J. Bard and L. R. Faulkner, 2001. Wiley, 2nd ed., 2000. 
[34] D. Harvey, "Analytical chemistry 2.0." Analytical Sciences Digital Library, 1999.

[35] R. G. Kazemzadeh S. and A. R., "Novel approach of biophotovoltaic solid state solar cells based on a multilayer of PS1 complexes as an active layer," ACS Sustainable Chemistry \& Engineering, vol. 5, no. 11, pp. 9836-9840, 2017.

[36] K. Nakata and A. Fujishima, "TiO $\mathrm{O}_{2}$ photocatalysis: Design and applications," Journal of Photochemistry and Photobiology C: Photochemistry Reviews, vol. 13, no. 3, pp. 169 - 189, 2012.

[37] W. R. Henson, V. B. Shah, G. Lakin, T. Chadha, H. Liu, R. E. Blankenship, and P. Biswas, "Production and performance of a photosystem I-based solar cell using nano-columnar $\mathrm{TiO}_{2}, "$ in 2013 IEEE 39th Photovoltaic Specialists Conference (PVSC), pp. 2705-2709, June 2013.

[38] J. Burschka, N. Pellet, S. J. Moon, R. Humphry-Baker, P. Gao, M. K. Nazeeruddin, and M. Gratzel, "Sequential deposition as a route to high-performance perovskite-sensitized solar cells," Nature, vol. 499, pp. 316-319, Jul 2013.

[39] O. Yehezkeli, R. Tel-Vered, J. Wasserman, A. Trifonov, D. Michaeli, R. Nechushtai, and I. Willner, "Integrated photosystem II-based photo-bioelectrochemical cells," Nat Commun, vol. 3, p. 742, Mar 2012 .

[40] J. O. Calkins, Y. Umasankar, H. O'Neill, and R. P. Ramasamy, "High photo-electrochemical activity of thylakoid-carbon nanotube composites for photosynthetic energy conversion," Energy Environ. Sci., vol. 6, pp. 1891-1900, 2013.

[41] P. J. Nixon, F. Michoux, J. Yu, M. Boehm, and J. Komenda, "Recent advances in understanding the assembly and repair of photosystem II," Ann. Bot., vol. 106, pp. 1-16, Jul 2010.

[42] A. Akbarzadeh, R. Rezaei-Sadabady, S. Davaran, S. W. Joo, N. Zarghami, Y. Hanifehpour, M. Samiei, M. Kouhi, and K. Nejati-Koshki, "Liposome: classification, preparation, and applications," Nanoscale Res Lett, vol. 8, p. 102, Feb 2013.

[43] E. Nénonéné and M. Fragata, "Interaction of photosystem $\{\mathrm{II}\}$ proteins with non-aggregated membranes constituted of phosphatidylglycerol and the electrically neutral phosphatidyl- 
choline enhances the oxygen-evolving activity," Chemistry and Physics of Lipids, vol. 91, no. 2 , pp. $97-107,1998$.

[44] S. C. Popat, D. Ki, B. E. Rittmann, and C. I. Torres, "Importance of OH(-) transport from cathodes in microbial fuel cells," ChemSusChem, vol. 5, pp. 1071-1079, Jun 2012.

[45] S. Pandit, S. Khilari, K. Bera, D. Pradhan, and D. Das, "Application of PVA-PDDA polymer electrolyte composite anion exchange membrane separator for improved bioelectricity production in a single chambered microbial fuel cell," Chemical Engineering Journal, vol. 257, no. Supplement C, pp. $138-147,2014$.

[46] Y.-E. Chen, S. Yuan, and W. P. Schröder, "Comparison of methods for extracting thylakoid membranes of arabidopsis plants," Physiologia Plantarum, vol. 156, no. 1, pp. 3-12, 2016.

[47] D. I. Arnon, "COPPER ENZYMES IN ISOLATED CHLOROPLASTS. POLYPHENOLOXIDASE IN BETA VULGARIS," Plant Physiol., vol. 24, pp. 1-15, Jan 1949.

[48] M. Fragata, Photosynthesis Research Protocols, vol. 274 of Methods in Molecular Biology, ch. Photosystem II Reconstitution Into Proteoliposomes, pp. 183-204. Humana Press, 2004.

[49] S. Eshaghi, B. Andersson, and J. Barber, "Isolation of a highly active PSII-LHCII supercomplex from thylakoid membranes by a direct method," FEBS Letters, vol. 446, no. 1, pp. 23-26, 1999.

[50] P. J. Morrissey, S. W. McCauley, and A. Melis, "Differential detergent-solubilization of integral thylakoid membrane complexes in spinach chloroplasts. Localization of photosystem II, cytochrome b6-f complex and photosystem I," Eur. J. Biochem., vol. 160, pp. 389-393, Oct 1986.

[51] T. M. Bricker, J. G. Metz, D. Miles, and L. A. Sherman, "Biochemical characterization of a highly active $\mathrm{O}_{2}$-evolving photosystem II preparation from maize," Biochimica et Biophysica Acta (BBA) - Bioenergetics, vol. 724, no. 3, pp. $447-455,1983$.

[52] U. K. Laemmli, "Cleavage of structural proteins during the assembly of the head of bacteriophage T4," Nature, vol. 227, pp. 680-685, Aug 1970. 
[53] N.-H. Chua, "[40] electrophoretic analysis of chloroplast proteins," in Photosynthesis and Nitrogen Fixation - Part C (A. S. Pietro, ed.), vol. 69 of Methods in Enzymology, pp. 434 - 446, Academic Press, 1980.

[54] H. Schagger, "Tricine-SDS-PAGE," Nat Protoc, vol. 1, no. 1, pp. 16-22, 2006.

[55] M. Chevallet, S. Luche, and T. Rabilloud, "Silver staining of proteins in polyacrylamide gels," Nat Protoc, vol. 1, no. 4, pp. 1852-1858, 2006.

[56] W. L. Nastuk, Physical Techniques in Biological Research, vol. IV: Special Methods, ch. 3The Oxygen Cathode. Elsevier Inc., 1962.

[57] R. Barr, M. D. Henninger, and F. L. Crane, "Comparative Studies on Plastoquinone II. Analysis for Plastoquinones A, B, C, and D," Plant Physiol., vol. 42, pp. 1246-1254, Sep 1967.

[58] R. B. Boers, Y. P. Randulfe, H. N. S. v. d. Haas, M. v. Rossum-Baan, and J. Lugtenburg, "Synthesis and spectroscopic characterization of 1-13c- and 4-13c-plastoquinone-9," European Journal of Organic Chemistry, vol. 2002, no. 13, pp. 2094-2108, 2002.

[59] Z. Wang and C. Benning, "Arabidopsis thaliana polar glycerolipid profiling by thin layer chromatography (TLC) coupled with gas-liquid chromatography (GLC)," J Vis Exp, Mar 2011.

[60] G. Nuzzo, C. Gallo, G. d'Ippolito, A. Cutignano, A. Sardo, and A. Fontana, "Composition and quantitation of microalgal lipids by ERETIC ${ }^{1} \mathrm{H}$ NMR method," Mar Drugs, vol. 11, pp. 3742-3753, Sep 2013.

[61] A. M. Becka and C. J. Miller, "Electrochemistry at $\omega$-hydroxy thiol coated electrodes. 3. voltage independence of the electron tunneling barrier and measurements of redox kinetics at large overpotentials," The Journal of Physical Chemistry, vol. 96, no. 6, pp. 2657-2668, 1992.

[62] S. Khilari, S. Pandit, D. Das, and D. Pradhan, "Manganese cobaltite/polypyrrole nanocomposite-based air-cathode for sustainable power generation in the single-chambered microbial fuel cells," Biosens Bioelectron, vol. 54, pp. 534-540, Apr 2014. 
[63] D. J. Davis and A. San Pietro, "Interactions between spinach ferredoxin and other electron carriers. The involvement of a ferredoxin:cytochrome $\mathrm{C}$ complex in the ferredoxin-linked cytochrome C reductase activity of ferredoxin:NADP + oxidoreductase," Arch. Biochem. Biophys., vol. 182, pp. 266-272, Jul 1977. 